\title{
Micropillar Arrays for High Sensitivity Sensors
}

\author{
by \\ Youngwoo Kim \\ A dissertation submitted in partial fulfillment \\ of the requirements for the degree \\ Doctor of Philosophy \\ (Mechanical Engineering) \\ in The University of Michigan \\ 2016
}

Doctoral Committee:

Associate Professor Nakhiah C. Goulbourne, Co- Chair

Professor Alan S. Wineman, Co- Chair

Professor Daniel J. Inman

Professor Jwo Pan 
(C) Youngwoo Kim 2016 
To my family. 


\section{Acknowledgements}

First of all, I would like to give my sincere appreciation to Dr. Nakhiah Goulbourne for her patience, support, and superb instructions. Her excellent guidance and immense knowledge on the field have shaped me to become a better scholar. It has been a great honor to have her as my advisor and mentor. I hope that I will be able to continuously seek her wisdom and advice even after I graduate.

I would also like to thank Dr. Wineman, Dr. Inman and Dr. Pan for being my committee. I deeply appreciate their support.

I would like to express my gratitude to for following members of the Smart Material Research Lab (SMRL) in University of Michigan: Alyssa, Riddhiman, Yali, Miki Lee, Alexander Van Kainen, and former SMRL students. The insightful inputs and valuable cooperation of my fellow SMRL colleagues have been instrumental in my success during graduate school.

I would also like to give many thanks to my parents for their love and support. They have been my solid bedrock throughout my years in University of Michigan, showing faith in me and giving me million words of encouragement.

Lastly, I want to thank and praise God for making all this possible. 


\section{Table of Contents}

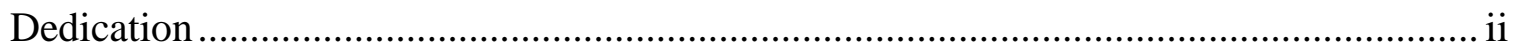

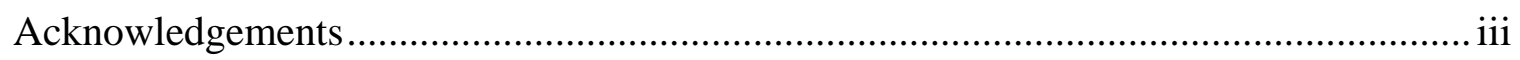

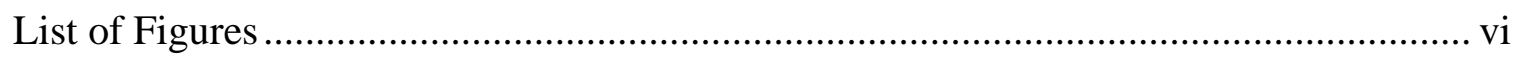

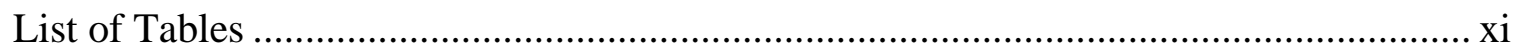

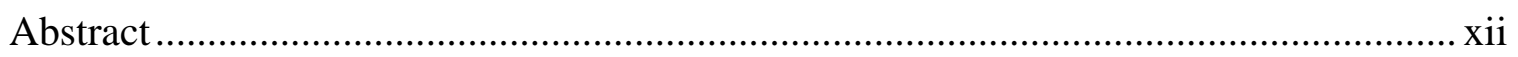

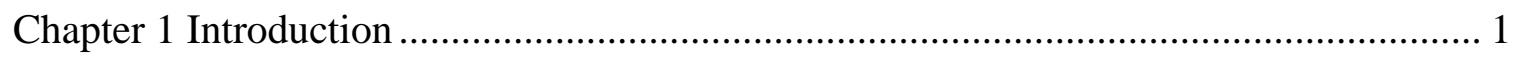

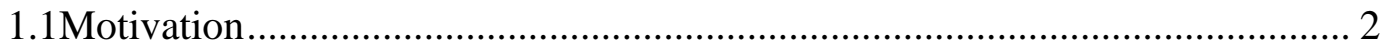

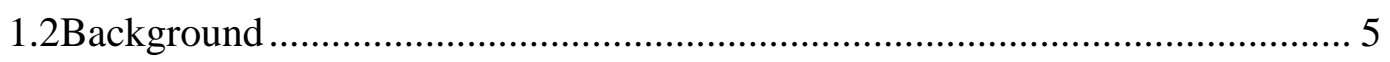

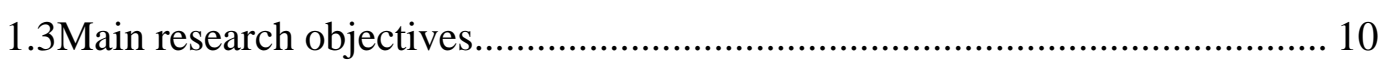

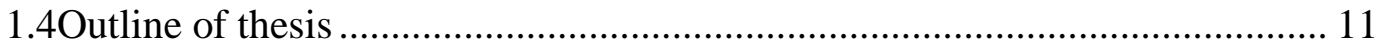

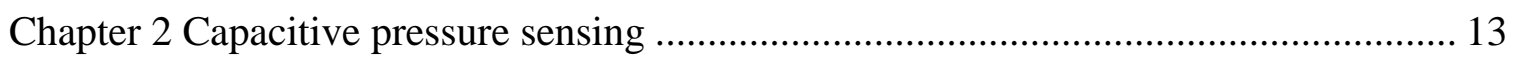

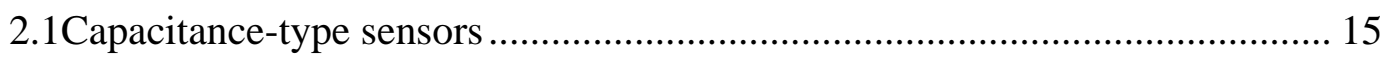

2.1.1Elementary laws of capacitors ........................................................ 16

2.1.2Capacitance change in an Electric field .......................................... 16

2.2Capacitance in a nonuniform electric field ........................................... 21

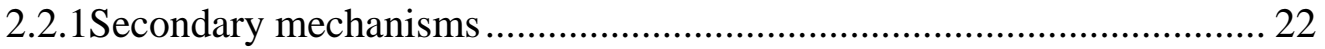

Chapter 3 Fabrication of PDMS Micropillar Arrays ............................................... 24

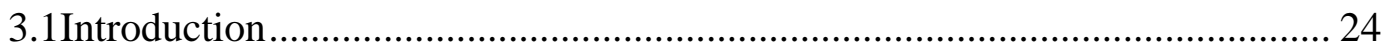

3.1.1Overview of materials and process selection.................................. 24

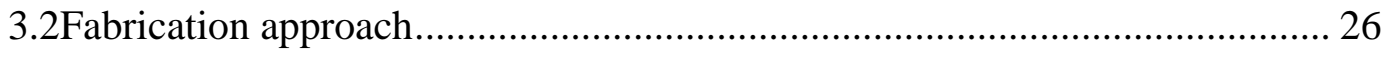

3.2.1PDMS micropatterning techniques ............................................. 28

3.2.2Deep Reactive Ion Etching (DRIE) ................................................ 29

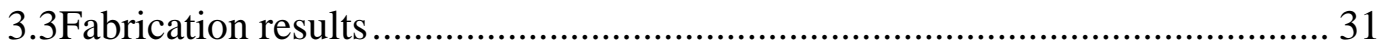

3.3.1Electroding technique ............................................................. 32

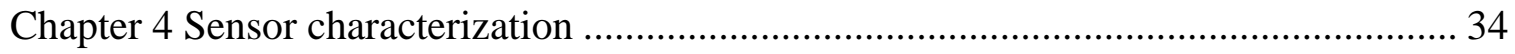




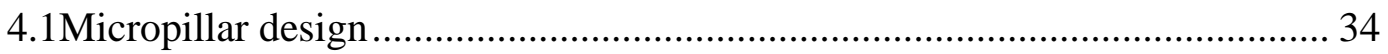

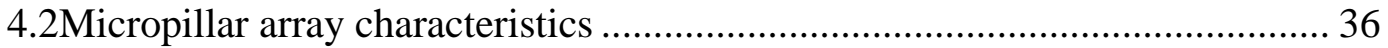

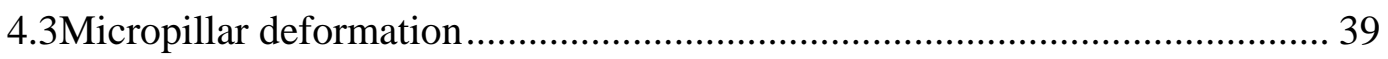

4.3.1Influence of pillar imperfection on the deformation ............................ 39

4.3.2Micropillar deformation of optimized pillar geometries ..................... 41

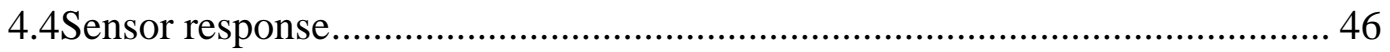

4.5Time scale characteristics of sensor response........................................... 52

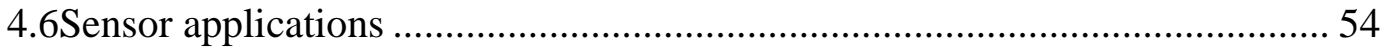

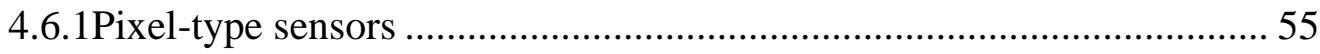

4.6.2Arterial blood pressure sensors ................................................... 56

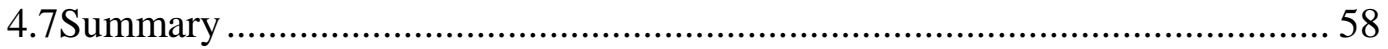

Chapter 5 Computational Analysis of Micropillar Sensor Arrays ................................. 60

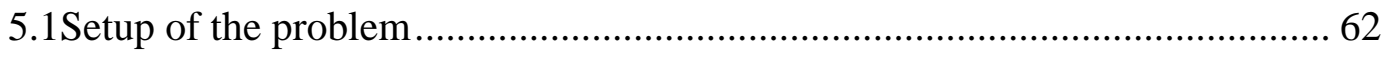

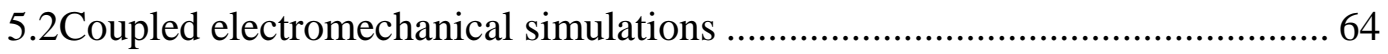

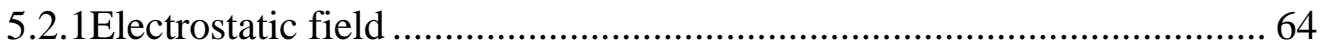

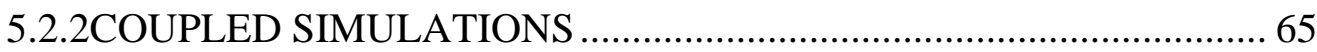

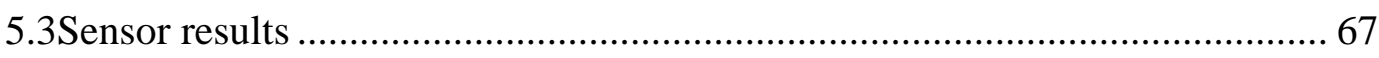

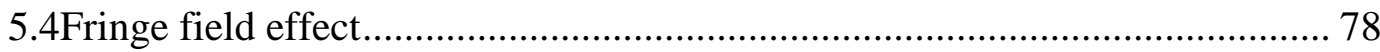

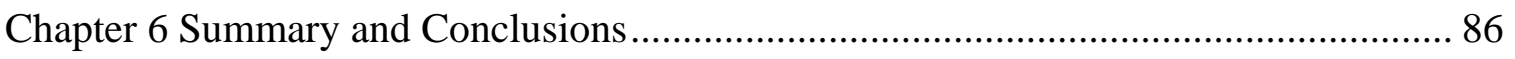

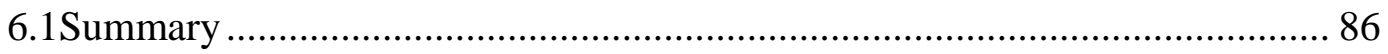

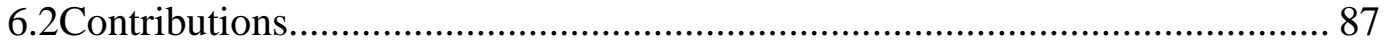

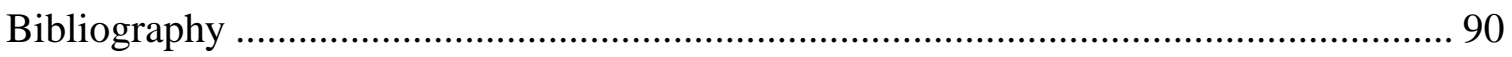




\section{List of Figures}

Figure 1.1 Photographs showing the sensor developed in this thesis: left) stretched sensor

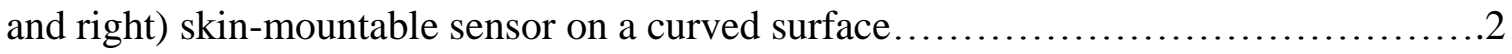

Figure 1.2 Ciliary structures as seen by SEM in the Lung Trachea $\ldots \ldots \ldots \ldots \ldots \ldots \ldots . . . \ldots$ Figure 1.3 Top) SEM images of the highly regular structures obtained via soft replica molding and (1:3 aspect ratio) Bottom) structural collapse due to mechanical instability

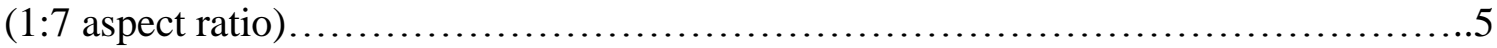

Figure 2.1 Micropillar array configuration and intended performance. a) closed micropillar array configured for capacitive pressure sensing (PDMS+ Au), b) closed micropillar array configured for actuation, c) open micropillar array for resistive flow sensing (conductive polymer $+\mathrm{Au}$ ), d) closed micropillar array configured for resistive

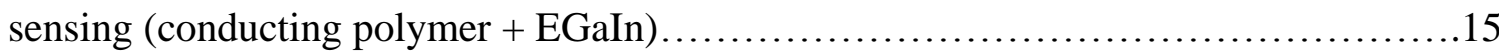

Figure 2.2 (left) PDMS micropillar array, (center) electroded monolithic film, and (right)

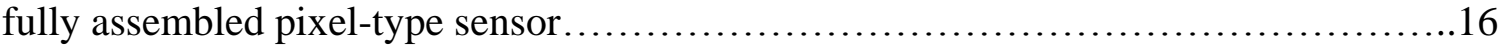

Figure 2.3 Dissimilar charges in close proximity and similar charges in close proximity illustrating Coulomb's Law.................................................... Figure 2.4 A simple parallel plate capacitor consisting of a monolithic dielectric and rigid monolithic electrodes and the resulting electric field domain.

Figure 2.5 Top: Basic principle of capacitive sensors due to change in the distance between the electrodes, d. left) undeformed micropillar sensor and right) deformed micropillar sensor. Bottom: Electric field of micropillar array ...................... 18

Figure 2.6 A simple parallel plate capacitor.................................... 19

Figure 2.7 Electric field simulation for a single pillar. Left) shaded electrode regions of a single pillar and right) The bulk of the field is contained in the monolithic film and gently extends into the pillar region and gap. Note the fringe fields at the edges of the PDMS film and on top of the Au electrode. 
Figure 2.8 Object interferes with the fringe field and changes the capacitance of the

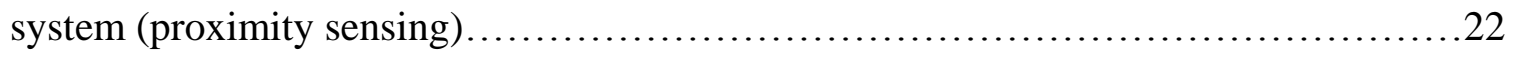

Figure 2.9 Changes in the electric field due to object placement.....................23

Figure 3.1 i) Fabrication of $\mathrm{Au}$ buckled electrodes. ii) Micrograph of buckled $\mathrm{Au}$ electrode. iii) Relative resistance versus uniaxial stretch for buckled Au electrode film..26 Figure 3.2 (a) Schematic of the PDMS molding process by replica modling. (b) SEM

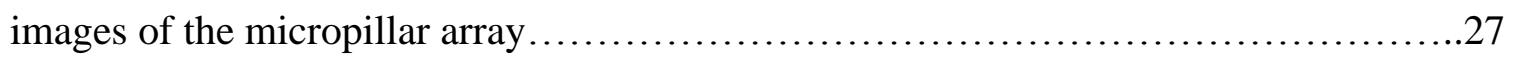

Figure 3.3 Schematic of electroding process and sensor assembly. (a) PDMS micropillar array layer (b) Au electroded pillar surface patterened into lines using a shadow mask $25 \mathrm{~mm}$ x 50mm (c) A top monolithic PDMS film placed on top of the Au micropillar array and is subsequently electroded using a shadow mask. The electroded lines form a grid pattern to create through thickness capacitive cells or pixels...................27

Figure 3.4 Hybrid mask design is a combination of AR 1:3,1:5,1:7 pillars on a single platform. left) SEM image of pattened SI wafer. right) Schematic of hybrid design......31 Figure 3.5 Fabrication steps for hybrid micropillar mold ..........................31 Figure 3.6 SEM images of as fabricated micropillar array surfaces a) $200 \mu \mathrm{m}$ gap 1:1

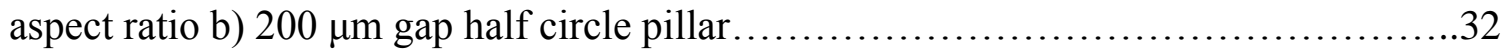
Figure 3.7 Individual pillars are uniformly coated with Au forming a thin nanoscale layer along the vertical walls, pillar top surfaces, and in the gaps between the pillars.... .33 Figure 3.8 Au electroded micropillar array and final assembly for capacitive pixel sensing .33

Figure 4.1 a) (Top) Test schematic and b) (bottom) Constant test area for the different

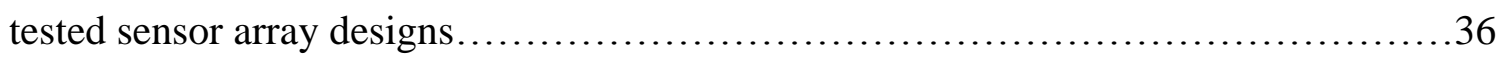

Figure 4.2 1:1 $100 \mu \mathrm{m}$ gap: square pillar, circle pillar, triangle pillar, half circle pillar...37

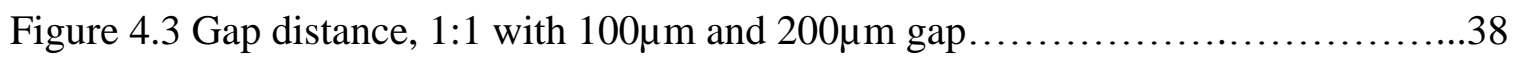

Figure 4.4. Aspect ratios from top to bottom - 1:3, 1:6, 1:7, and 1:9................. 39

Figure 4.5 The pillar surface is not flat and planar................................ 40

Figure 4.6 Tilting and bending of pillars - experimental results $\ldots \ldots \ldots \ldots \ldots \ldots \ldots \ldots . .41$

Figure 4.7 Tilting and bending of pillars - model simulation results showing stress (left) and strain (right) fields 
Figure 4.8 Progression of the deformation in low aspect ratio pillars and a schematic

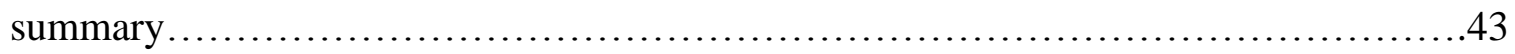

Figure 4.9 Top down picture (left) and schematic (right) of hybrid pillar array..........44 Figure 4.10 Optical images tracking the evolution of the deformation in C-shaped pillars under pressure loading. The arrows in the bottom schematic denote the viewpoint of the

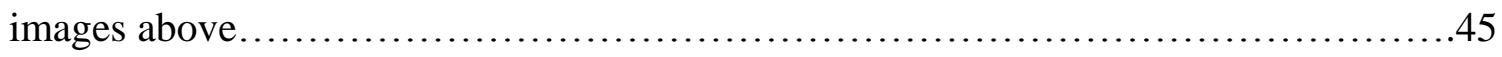

Figure 4.11 Cyclic response of sensor array when a $5 \mathrm{~g}$ weight is applied..............47

Figure 4.12 Sensor response for different cross-sections (constant area)...............48

Figure 4.13 Sensor capacitance response for three different pillar aspect ratios..........49

Figure 4.14 Sensor capacitance response for three different Half circle designs..........50

Figure 4.15 Sensor capacitance response for Hybrid with three different pillar aspect ratios.

Figure 4.16 Circuit used to measure sensor response time taken from Ref [83] .53

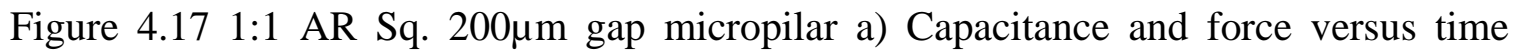
graph. B) Capacitance versus time graph. The response time is $60.8 \mathrm{~ms} . \ldots \ldots \ldots \ldots . . .54$

Figure 4.18 Multiplex processing circuit for multi-capacitor array readout............55

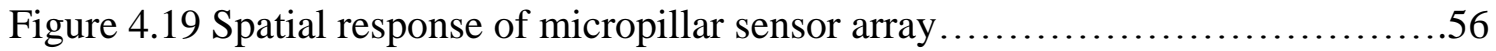

Figure 4.20 Arterial blood pressure by wrist measurement for subject at rest...........57

Figure 5.1 Problem formulation in ABAQUS and COMSOL ......................61

Figure 5.2 The nonuniform electric field due to spatially varying permittivity and fringe field effects of a single pillar. The electric field is overlaid on the deformed pillar geometry. Note that the bulk of the field is contained in the PDMS monolithic layer...62 Figure 5.3 Comparison of the stress and displacement fields in the thickness direction and the overall displacement of a single pillar from ABAQUS and COMSOL.............64 Figure 5.4 Convergence test for capacitance calculation performed by studying the effect of air domain size on the fringe field whilst keeping the mesh constant. Plot of relative capacitance as a function of domain size for different levels of loading...............65

Figure 5.5 Geometrical aspects of the pillar designs simulated and tested

Figure 5.6 Increasing the number of pillars increases the sensitivity. There is a very good match with experimental results when the entire array is replicated .68 
Figure 5.7 Computational results for sensor output for the different designs

Figure 5.8 Overall geometry and strain of single pillar (top) mechanical stress and strain fields (middle) and electric field distribution (bottom)........................... 70

Figure 5.9 Overall geometry and displacement of $10 \times 10$ array (top) mechanical stress and strain fields (middle) and electric field distribution (bottom). .71

Figure 5.10 Overall geometry and displacement of $25 \times 25$ array (top) mechanical stress and strain fields (middle) and electric field distribution with zoomed in view(bottom)..72 Figure 5.11 Geometry of 50 x 50 array (top) mechanical stress and strain fields (middle) and electric field distribution with zoomed in view (bottom) ........................ 73

Figure 5.12 Zoomed in views of electric field as a function of the number of pillars in the simulated array. 74

Figure 5.13 Simulation results for the $25 \times 25$ array with varying aspect ratios. Mechanical stress and strain fields (top) and electric field distribution with zoomed in view (bottom). .75

Figure 5.1425 x 25 array with varying gap distances. Mechanical stress and strain fields (top) and electric field distribution with zoomed in view (bottom)... .76

Figure 5.15 Simulation results for a $25 \times 25$ array with 3 varying cross-sections, full circle, thick-walled half circle, and thin-walled half circle compared to a square 1:1 array. Half circle pillar array geometry (top). Mechanical stress and strain fields (middle) and

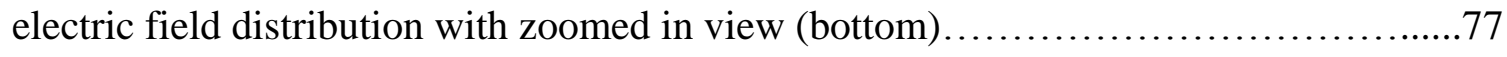

Figure 5.16 Meshed configuration for the proximity test..........................79 Figure 5.17 Simulations for a conducting object approaching sensor surface. Relative permittivity set to 10000 to simulate a conducting object in COMSOL. a) No contact with sensor and a10 $\mu \mathrm{m}$ gap, b) contact with sensor. Relative permittivity set to 100 to simulate a conducting object in COMSOL. d) No contact with sensor and a10 $\mu \mathrm{m}$ gap, and e) contact with sensor...................................................... 80

Figure 5.18 Simulations for a dielectric object approaching sensor surface. Relative permittivity set to 10:a) No contact with sensor and a10 $\mu \mathrm{m}$ gap,b) contact with sensor.81

Figure 5.19 Initial sensor capacitance for each test case. 
Figure 5.20 Relative capacitance change normalized with respect to sensor only

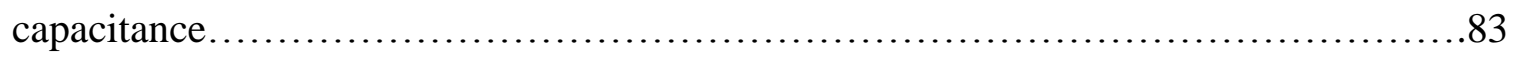

Figure 5.21 Relative capacitance change as a function of pressure in the presence of a dielectric or conducting object............................................... 83 Figure 5.22. Relative capacitance change as a function of pressure in the 1-10 Pa

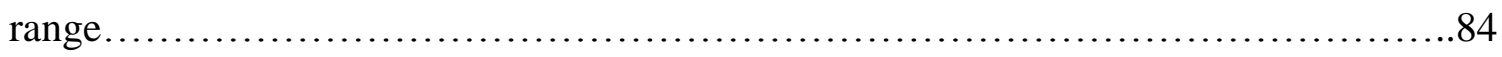




\section{List of Tables}

Table 1.1 Micropillar Sensor Summary ........................................ .

Table 1.2 Summary of target sensor characteristics........................... 10

Table 2.1 Advantages and disadvantages of pressure sensors.......................13

Table 3.1 Summary of sample dimensions fabricated..............................28

Table 4.1 Geometric specifications for all devices fabricated and tested...............35

Table 4.2 Nanofabrication accuracy before improving fabrication process.............40

Table 4.3 Mechanism and Functionality of Micropillar Sensor Arrays...................46

Table 4.4 Sensitivity (kPa-1) in the different pressure regimes for each of the designs..52

Table 4.5 Comparison of the proposed sensor and other flexible sensor systems........54

Table 4.6 Comparison of microstructured sensors................................59

Table 5.1 Material properties for COMSOL simulations..........................63

Table 5.2 Test matrix for COMSOL simulations................................66

Table 5.3 Sensitivity results for the different array designs (simulation results).........69

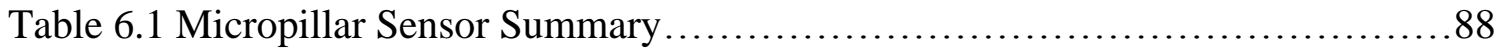




\begin{abstract}
In this thesis, a soft and sensory skin-like electronic structure is developed. A capacitance-type pressure sensor is developed that is not only flexible but also stretchable, has a wide sensing regime (up to $10 \mathrm{kPa})$, and high sensitivity $\left(0.34 \mathrm{kPa}^{-1}\right)$. Micropillar sensor arrays inspired by cilia structures found in nature are fabricated using a soft nanolithography technique and electroded with sputtered gold. The arrays are configured for capacitance type pressure sensing in the tactile - touch regime. Several designs are proposed, fabricated, and evaluated to optimize sensitivity and detectability in the low pressure range. Techniques for fabricating asymmetric easy to buckle pillar structures and a multi-level hierarchical design platform are developed. Here, we present the highest reported sensitivity $\left[0.34 \mathrm{kPa}^{-1}\right]$ of a passive capacitance type sensor that is both flexible and stretchable. There is experimental evidence to suggest that the sensor can be configured to detect very low pressures, and or used for proximity sensing.
\end{abstract}

The influence of pillar design parameters on sensor performance is explored using experimental and computational simulation techniques. The relationship between different pillar deformation modes and sensor characteristics is established and quantified. Computational simulations are carried out in COMSOL to investigate how large conformational changes of the pillars during deformation influence the capacitance readings for the different sensor designs. The simulations are an important tool in solving coupled multiphysics problems and for visualizing complex nonuniform three dimensional electrostatic fields. The simulation results show identical trends to the experiments and excellent correlation is achieved for the full device model. Finally, the microstructured sensor array naturally lends itself to the development of pixel-type pressure sensors and biomedical monitoring devices - the potential for both applications are demonstrated here. 


\section{Chapter 1}

\section{Introduction}

The interest in flexible and stretchable electronic systems capable of transducing mechanical, chemical, and or thermal signals has garnered attention in recent years [1-8]. From artificial intelligence to medical devices such as bio-implantable systems, there are various applications where classical rigid systems are neither desirable nor feasible. Flexible and stretchable devices have the potential to be directly wrapped, integrated, and or embedded around non-planar and curved geometries that stretch without compromising performance during the operating lifetime. These conformable devices will enable "feel" sensing through signals of touch, motion, flow, and pressure. It is desirable to enable these sensibilities on a single platform that can also respond appropriately i.e. an autonomic response to the feedback through actuation. Lightweight and wearable pressure sensors have many target applications such as electronic skins, soft robotics, prosthetics, wearable medical devices, multifunctional skins for UAV morphing wings, flexible displays, adaptive braille interfaces, and interactive tactile media for a host of sensory related applications [9-16]. In this thesis, a capacitance-type pressure sensor was developed that is not only flexible but also stretchable, has a wide sensing regime (up to $10 \mathrm{kPa}$ ), and high sensitivity $\left(0.34 \mathrm{kPa}^{-1}\right)$.

The system developed in this research is fabricated using a soft polymer (polydimethylsiloxane - PDMS) and sputtered gold (Au) electrodes. Specifically, the sensor device consists of a PDMS micropillar structure inspired by cilia found in biological organisms. Cilia are high aspect ratio structures that play an important role in multi-stimulus sensing, locomotion and propulsion in many living organisms [17]. The 
basic principle of sensor operation involves directly electroding the micropillars and forming a capacitive sensor configuration. This platform can easily be adapted for other functionalities such as resistive sensing and actuation. Because of the array microstructure, the local electric field is nonuniform; large deformations of the pillar network changes the local electrostatic field. In the current setup, an applied pressure load causes large film and pillar deformation and in certain cases large conformational changes of the pillars. These deformations change the microstructured gaps between the electrodes and hence the device capacitance. The small scale of the capacitive sensor also renders the fringe field non-negligible, which is believed to play a role in the improved sensitivity. Two applications are considered to illustrate viability of the sensors developed herein: i) a pixel-type pressure sensor and ii) a continuous monitoring device for arterial pressure fluctuations. It is anticipated that the sensor platform can be easily adapted to incorporate multiple functionalities in the future.

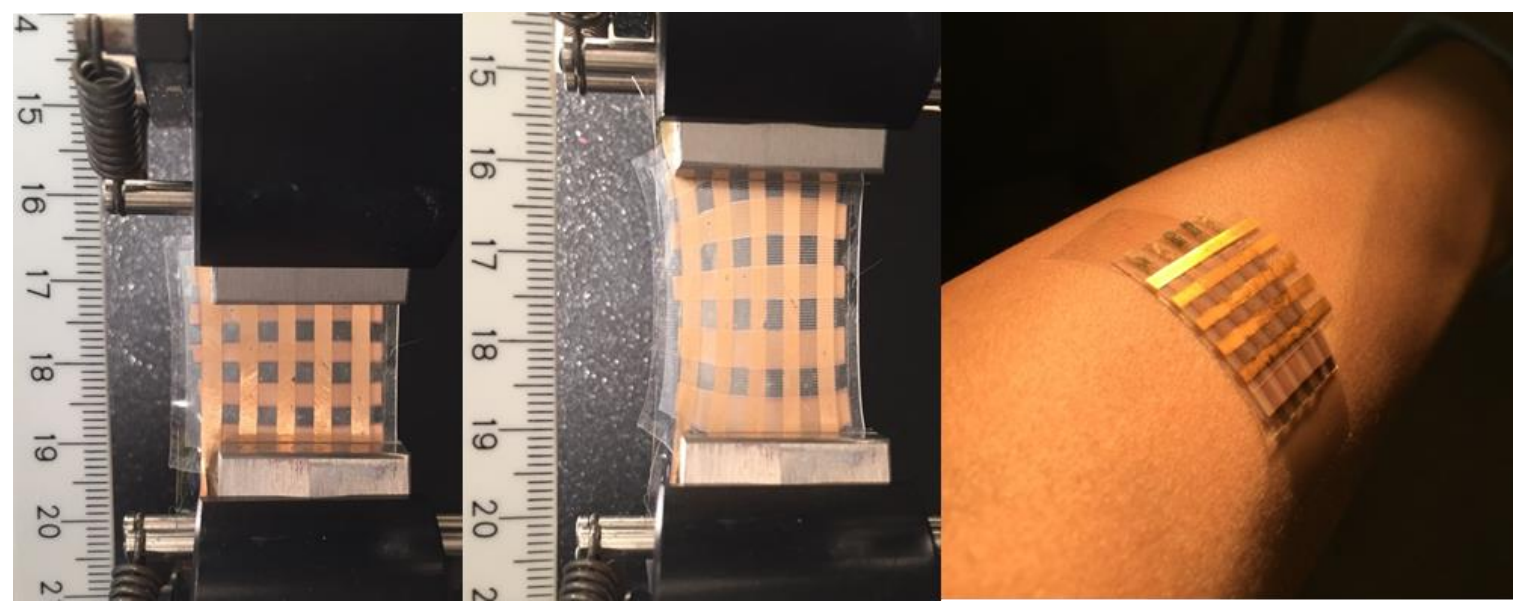

Figure 1.1 Photographs showing the sensor developed in this thesis: left) stretched sensor and right) skin-mountable sensor on a curved surface.

\subsection{Motivation}

In the scheme of living organisms, the role of cilia is a major one. Cilia are high aspect ratio multifunctional microstructures (Figure 1.2). Life functions are either completely dependent on ciliary systems or their efficiency is much improved because of them [18]. These microstructures are typically found interacting with fluids in biological channels. 
There are two kinds of cilia: primary cilia and motile cilia. Primary cilia are found on nearly every cell in the body as a sensory organelle. Motile cilia are self-propelling structures with a dominant presence in the lungs, pulmonary tract, and ear canal. The primary cilia interact with the extracellular environment and serve as cell-level sensory antennae for mechanical, thermal, and chemical input. From a biological perspective, the interaction of external stimuli with the sensing organelles induce mechanical reconfigurations, which is a key part of biological regulation and adaptation.

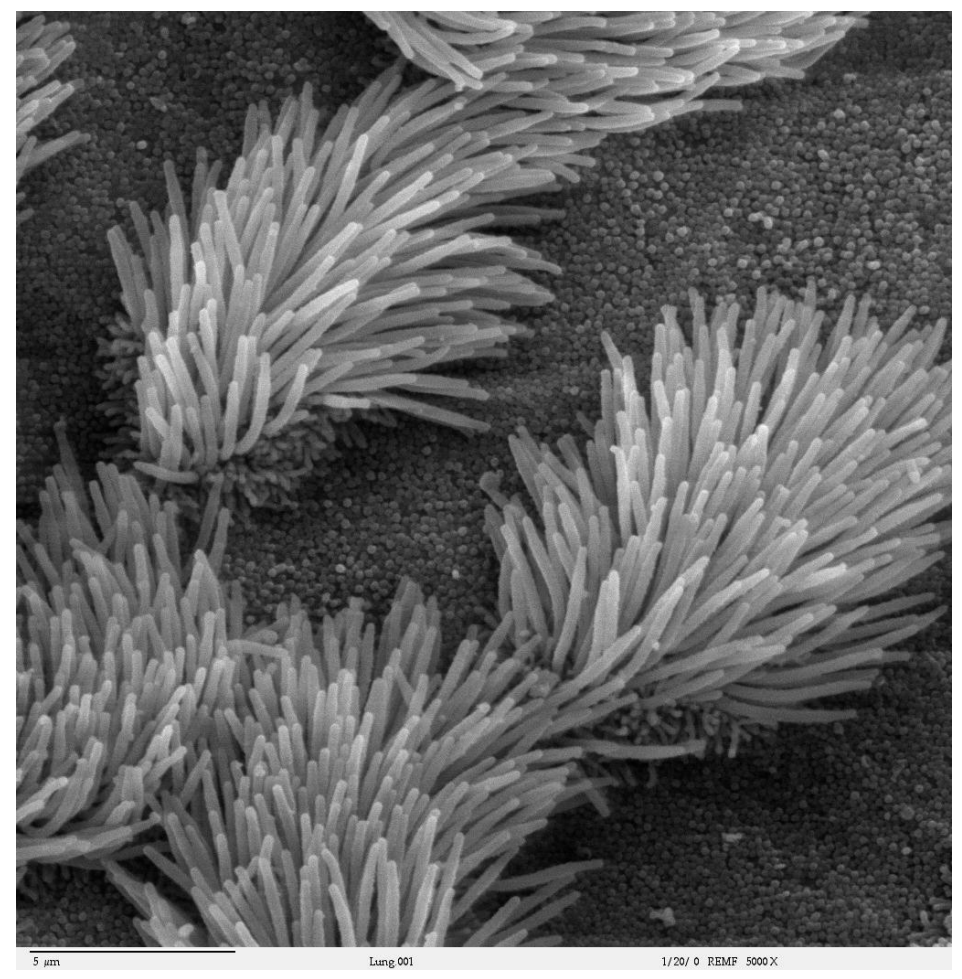

Figure 1.2 Ciliary structures as seen by SEM in the Lung Trachea [19].

Inspired by these biological microstructures, several research groups have developed polymer micropillar arrays for a range of target functionalities including but not limited to wetting control, optomechanical coupling for structural color change properties and varying transparency, chemomechanical actuation, electromechanical actuation, thermomechanical actuation, resistive strain sensing, capacitive pressure sensing, superhydrophobicity, drag reduction, and gecko-like adhesion [20-26]. The ultimate goal 
in some of these cases is to develop a singular platform for structures that sense and respond via structural reconfigurations.

The best-known method for fabricating soft micropillar arrays is soft lithography, which relies on an elastomeric mold to replicate the pattern. The main prerequisite for creating a desired geometry is a detailed and accurate master, which is typically fabricated by photolithography[27]. Photolithography uses a photoresist (SU-8), which has been specially developed to obtain high aspect ratio features. Arrays of pillars of radii between 1 and 100 microns and lengths of 5 to 100 microns have been reported [28] over $\mathrm{cm}$ scale areas of the silicon wafer. The aspect ratio of the pillars is limited by mechanical instability of micron sized PDMS with aspect ratios greater that 1:6. Pillar structure informs functions such as level of adhesion. Previous work has shown that 3-D geometry of the tips can be obtained either by the mold design or in post-mold processing steps during the cure phase leading to increased adhesion. Soft micropillar arrays by nanolithography have been utilized by an extensive number of researchers for various applications [29]. For example, the Aizenberg group has streamlined the PDMS mold technique to fabricate micro- and nano- scale pillars of epoxy, PU, PDMS, and hydrogel and investigate chemo-mechanical couplings, optomechanical properties, and hydrophobic surfaces [30-32]. Others have fabricated nanoscale PDMS pillars for strain gage applications [33-34].

The current work focuses on an all polymer micropillar arrays fabricated by soft lithography and covered with $\mathrm{Au}$ (by sputter coating) for pressure sensing. For completeness, it should be noted that there have been several reports on pillar, fibrillar, and hair-cell like structures made of electroactive materials such as ionic polymers and piezoelectric fibers fabricated and or grown via other methods [35-36]. Those active structures are outside the scope of the proposed work and not considered for direct context or comparison. 

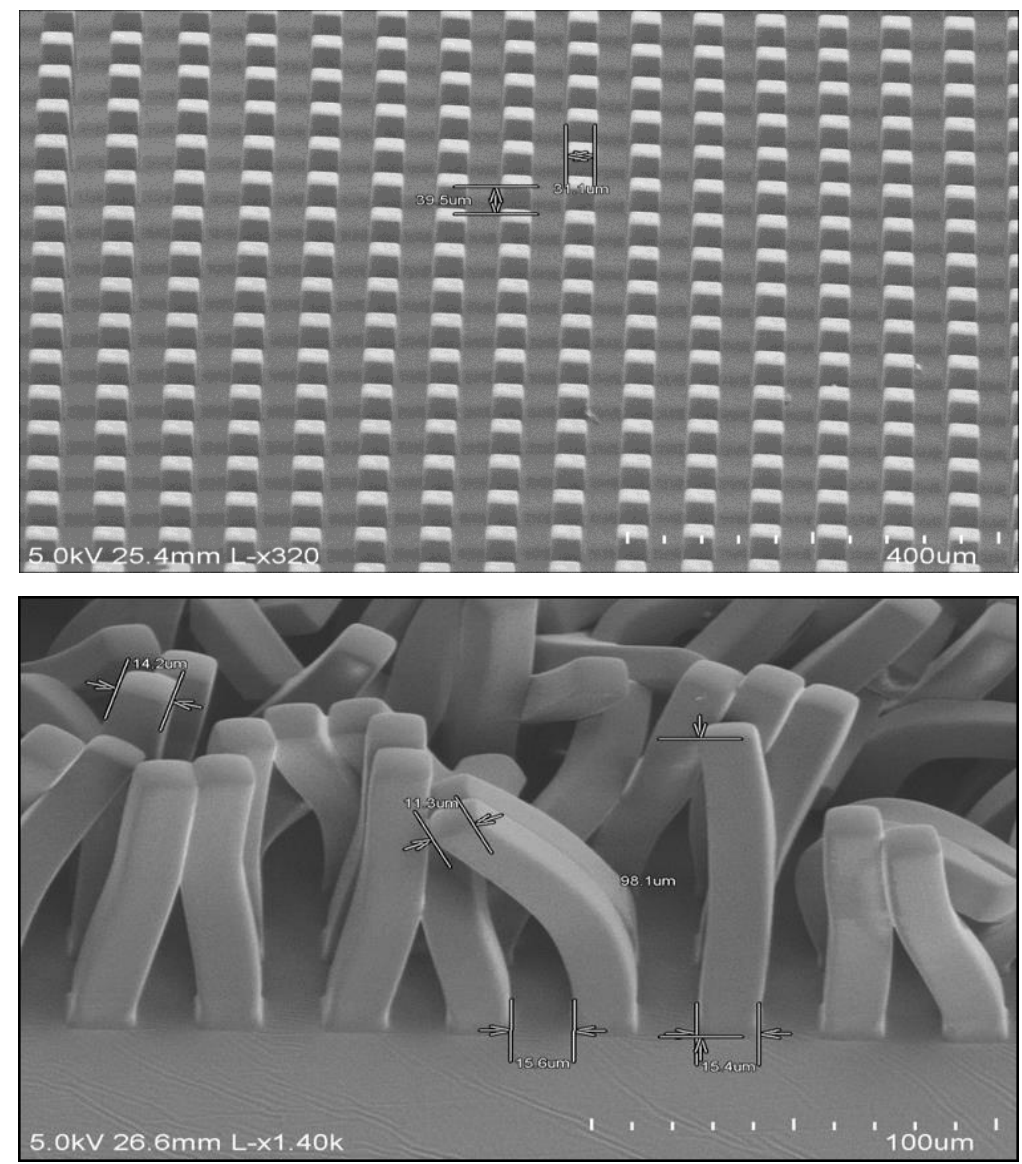

Figure 1.3 Top) SEM images of the highly regular structures obtained via soft replica molding and (1:3 aspect ratio) Bottom) structural collapse due to mechanical instability (1:7 aspect ratio).

\subsection{Background}

So-called touch or tactile sensors and soft pressure sensors have several challenges that impede their technological advancement. Touch sensors require force detection in multiple directions plus shear as well as high sensitivity at low pressures $(<50 \mathrm{kPa})$. It is desired that the sensors so-developed be amenable to large area deployment, and fabricated using low cost methods with the potential for scale up in addition to being flexible and stretchable. Organic pressure sensors have high accuracy but are either inflexible or have limited flexibility with no extensibility and so are generally a poor fit $[28,37]$. Capacitive sensors based on a polymer film have shown good potential as deformable sensors that can perhaps overcome the aforementioned challenges. 
Various techniques have been employed to develop soft sensors that address one or more limitations of the current state of the art. It is required that both the dielectric material (and or sensing element) and the conductor material are flexible and extensible. Several options exist for selecting a soft dielectric medium, though PDMS and PU are the most common. The choice for stretchable electrodes is the more limiting factor. Metallic films on a soft polymer can be mechanically configured to 'stretch' by inducing mechanical buckling, dense circuitous patterning, or controlled fracture [Lacour et al. 2005, Urdaneta et al. 2007]. Similar concepts have been demonstrated using carbon nanotube and graphene elements on a soft substrate or by dispersal in a polymer medium [40]. Aligned and randomly deposited nanotubes have demonstrated axial stretches of $100 \%$ and up to 700\%, respectively [Lipomi, Nature Nanotechnology 2011]. Electrodes like carbon grease and silver grease have been successfully used as actuator electrodes for dielectric elastomers undergoing stretches $~ 100 \%$ [Fox \& Goulbourne JMPS]. These electrodes however are messy to handle and not environmentally stable over long periods of time. They are also not easily adapted to existing nanofabrication techniques used to create discrete electrodes at small length scales. More recently, E Gallium Indium (EGa) has emerged as a liquid metal electrode with great promise for flexible and stretchable electronic devices and interfaces [Park, Wang Li et al 2012,]. Having a low solid to liquid transition point and its electro-osmotic properties has made it a viable option for electrode patterning of microscale and nanoscale devices [44]. As a whole, these mechanisms have successfully provided stable electrodes that are stretchable and flexible.

State of the art deformable pressure sensors are collectively made of: i) conductive polymer composites, ii) all polymer systems, iii) metal-impregnated elastomers, iv) composites made from carbon nanotubes, graphene, metallic particles, semiconducting nanowires, and metallic nanowires, and v) hybrid compositions integrating thin film organic semiconductors and piezoelectric films or particles [1,45-49,89]. Piezoelectric pressure sensors have high sensitivity but are often on the most rigid platform and cannot measure static loads. Conducting polymer sensors with few exceptions are not suitable for the ultra low-pressure regime and have problems with hysteresis and sensitivity in 
that range [89]. Graphene and carbon nanotube based sensors are costly and require highly specialized fabrication processes. Organic transistors are active devices that rarely suffer from environmental instability due to parasitic capacitance and drift to local impurities and contaminants as may be the case for microstructured all polymer passive devices. On the other hand, they are inherently rigid and suffer from hysteresis when coupled with pressure sensitive rubber substrates (to impart stretchability to the device). Pairing the Organic field-effect transistor (OFET) with a microstructured dielectric layer removes the hysteresis problem and produces a robust flexible device [Mannsfeld Nature materials, 2010]. High sensitivity pressure sensors were also fabricated by Shao et al based on an elastic ultrathin conductive polymer film (polypyrrole PPy) placed in contact with a Au covered micropillar polymer array [28]. They demonstrated the ability to tune the sensitivity from $0.03 \mathrm{kPa}^{-1}$ to $17 \mathrm{kPa}^{-1}$ by varying pillar diameter. The underlying mechanism boosting the sensitivity in the low pressure regime was contact resistivity between the PPy layer and the Au micropillar surface. Holm's theory shows a power law dependence of the contact resistance with external force, which was successfully realized through the sensor design. The sensor response is inherently nonlinear. Gong et al. developed a low cost fabrication procedure to make a highly flexible pressure sensor based on ultrathin gold nanowires embedded in tissue paper sandwiched between two PDMS sheets[52]. A similar concept was demonstrated by combining silver nanoparticles and elastomeric fibers to create highly stretchable and conductive polymer mats [Park,Im,Shin et al. 2012]. Using force induced physical contact between nanowire nets as the sensing mechanism, they reported a sensitivity of $1.14 \mathrm{kPa}^{-1}$ comparable to organic transistor pressure sensors. The resulting system is flexible though and not stretchable. The high cyclic stability of the device (> 50,000 cycles) is noteworthy as cyclic repeatability is a problem for elastomeric devices undergoing large stretches. In this regard, flexible sensor systems currently have the advantage.

Charalambides and Bergbreiter developed an all elastomer MEMS capacitive sensor using in plane interdigitated electrodes of a Ag/PDMS blend, which increased sensitivity by over an order of magnitude in comparison to using flat planar electrodes. Sensor 
accuracy up to $15 \%$ strain was reported in that work. Codd et al. used photolithography to fabricate helical pressure sensor pads consisting of microchannels filled with conductive liquid metal [55]. The pressure sensing skin was developed to detect tissue damage during neuroendoscopy. The sensor mechanism tracks resistance changes in the encapsulated liquid metal electrode when the channels are deformed by external pressure. The sensor is stretchable though in operation strains were not quantified in the work. Several other resistance type pressure sensors based on liquid metal embedded elastomer systems have been reported [56]. Park, Majidi, Kramer, et al. 2010, investigated several creative designs based on microchannels filled with liquid metal, but the sensor resolution was only $1 \mathrm{kPa}$ for a 1-100 $\mathrm{kPa}$ measuring range [57]. Yao and Zhu 2014 developed a multifunctional sensor platform using crumpled silver nanowires screen printed on a stretchable polymer substrate (Ecoflex). The sensors are capacitive and can detect strains up to 50\% and are operable over a range of pressures from the touch regime up to 1.2 MPa. The response is bilinear, and the sensor has a fast response time $(\sim 40$ $\mathrm{ms})$.

The area of flexible and stretchable electronics and sensors is quite broad and it would be difficult to exhaust all aspects in a single summary. In the preceding paragraphs, a sampling of research efforts and accomplishments using various approaches has been provided. Here, we narrow the focus to microstructured sensing systems for comparison with the proposed all polymer micropillar array developed in this thesis. The benchmark for microstructured pressure sensors is summarized in Table 1.1. The last row denotes the contribution from the research presented in this thesis. The list is by no means exhaustive and simply highlights some of the best features in systems proposed to date. Both high performing flexible and rigid microstructured systems are considered. 
Table 1.1 Micropillar Sensor Summary

\begin{tabular}{|c|c|c|c|c|c|c|}
\hline Type & Structure & $\begin{array}{c}\text { Sensing } \\
\text { mechanism }\end{array}$ & $\begin{array}{c}\text { Fabrication } \\
\text { technique }\end{array}$ & $\begin{array}{l}\text { Sensitivity } \\
\left(\mathrm{kPa}^{-1}\right)\end{array}$ & Range & $\begin{array}{c}\text { Response } \\
\text { time }\end{array}$ \\
\hline Flexible & $\begin{array}{l}\text { Gold serpentine } \\
\text { pattern on PDMS }\end{array}$ & Resistive & $\begin{array}{c}\text { PDMS molding } \\
\text { with Al mold }\end{array}$ & 0.23 & $\begin{array}{c}1 \mathrm{~Pa} \sim \\
6.7 \mathrm{kPa}\end{array}$ & $60 \mathrm{~ms}$ \\
\hline Flexible & $\begin{array}{l}\text { OFETs - indium tin } \\
\text { oxide (ITO)-coated } \\
\text { flexible PET sheet } \\
\text { on top of } \\
\text { microstructured } \\
\text { PDMS }\end{array}$ & Capacitive & $\begin{array}{c}\text { Photolith. for SI } \\
\text { mold, PVD of } \\
\text { Rubrene }\end{array}$ & $\begin{array}{c}0.55 \\
(<2 \mathrm{kPa} \\
\text { regime })\end{array}$ & $\begin{array}{l}3 \mathrm{~Pa} \sim \\
7 \mathrm{kPa}\end{array}$ & $\sim 200 \mathrm{~ms}$ \\
\hline Flexible & $\begin{array}{l}\text { Ppy gel (conducting } \\
\text { polymer) }\end{array}$ & Piezo-Resistive & $\begin{array}{c}\text { Micropatterned } \\
\text { inverse mold }\end{array}$ & $\begin{array}{l}56-133 \\
(<30 \mathrm{~Pa} \\
\text { regime })\end{array}$ & $\begin{array}{l}0.8 \mathrm{~Pa} \sim \\
10 \mathrm{kPa}\end{array}$ & $50 \mathrm{~ms}$ \\
\hline Flexible & $\begin{array}{l}\text { Reversible } \\
\text { interlocking of } \\
\text { nanoofibres }\end{array}$ & Piezo-Resistive & $\begin{array}{c}\text { SI Master, PUA } \\
\text { mold }\end{array}$ & $\begin{array}{l}11.45 \\
\text { (Gauge } \\
\text { factor) }\end{array}$ & $\begin{array}{l}5 \mathrm{~Pa} \sim \\
1.5 \mathrm{kPa}\end{array}$ & N/A \\
\hline Rigid & $\begin{array}{l}\text { parylene-based } \\
\text { wireless pressure } \\
\text { sensor }\end{array}$ & $\begin{array}{c}\text { Capacitor/indu } \\
\text { ctor resonant } \\
\text { circuits }\end{array}$ & $\begin{array}{c}\text { Silicon based } \\
\text { micromachined } \\
\text { (DRIE) }\end{array}$ & 0.058 & $\begin{array}{c}100 \mathrm{~Pa} \sim \\
4 \mathrm{kPa}\end{array}$ & N/A \\
\hline Rigid & $\begin{array}{c}\text { micropillar } \\
\text { structure on } \\
\text { conductive PPy } \\
\text { film }\end{array}$ & Resistive & $\begin{array}{l}\text { PDMS molding } \\
\text { with SI mold }\end{array}$ & $0.03-17$ & $\begin{array}{l}2 \mathrm{~Pa} \sim \\
3 \mathrm{kPa}\end{array}$ & $\sim 1000 \mathrm{~ms}$ \\
\hline $\begin{array}{l}\text { Stretchable } \\
\text { up to } 150 \%\end{array}$ & $\begin{array}{l}\text { Stretched CNT on } \\
\text { PDMS, }\end{array}$ & $\begin{array}{c}\text { Capacitive and } \\
\text { Resistive }\end{array}$ & $\begin{array}{c}\text { Spray coating of } \\
\text { CNT, multiple } \\
\text { layer stack }\end{array}$ & $2.3 \times 10^{-6}$ & $50 \mathrm{kPa} \sim$ & $<125 \mathrm{~ms}$ \\
\hline Flexible & $\begin{array}{c}\text { polymer } \\
\text { semiconductor/PD } \\
\text { MS dielectric }\end{array}$ & Capacitive & \begin{tabular}{|c|} 
assembling \\
separate layers \\
via lamination
\end{tabular} & $\begin{array}{c}8.4 \\
(<8 \mathrm{kPa})\end{array}$ & $50 \mathrm{~Pa} \sim$ & $<10 \mathrm{~ms}$ \\
\hline $\begin{array}{c}\begin{array}{c}* \text { Flexible } \\
\text { and } \\
\text { Stretchable } \\
\text { up to } 50 \%\end{array} \\
\end{array}$ & $\begin{array}{c}\text { Au coated micro } \\
\text { pillar structure on } \\
\text { PDMS film }\end{array}$ & Capacitive & $\begin{array}{c}\text { Soft } \\
\text { lithography }\end{array}$ & 0.34 & $\begin{array}{l}\text { 80Pa } \\
10 \mathrm{kPa}\end{array}$ & 60.8 \\
\hline
\end{tabular}

* The current thesis research results reported here.

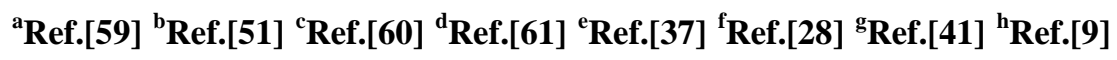

Liu et al [35], Mannsfeld et al [51], and D. Lipomi et al [41] all introduced microstructured thin film systems showing stretchability, a wide pressure sensing range, and 
high sensitivity (see Table 1.1). An important feature of Mannsfeld et al.'s work is that hysteresis in the polymer dielectric was reduced as a result of the microstructured architecture (because of the air gaps) in comparison to a monolithic layer. This is one advantage of using microstructured elastomers. Of the capacitive sensors proposed to date, the highest reported sensitivity is $8.4 \mathrm{kPa}^{-1}$ and that was obtained using a field effect transistor element to develop a flexible sensor. Low detectable pressures of $<5 \mathrm{~Pa}$ were reported whilst maintaining wide measuring ranges up to $\sim 10 \mathrm{kPa}$ (Table 1).

\subsection{Main research objectives}

The primary objective of this work is to achieve a skin level sensor system using existing fabrication techniques. Specifically, a flexible, stretchable sensor with high sensitivity, good spatial resolution, and a large sensing range is proposed and developed. The target sensor requirements are summarized in Table 1.2.

Table 1.2 Summary of target sensor characteristics.

\begin{tabular}{|c|c|}
\hline Property & Target characteristics \\
\hline Flexible & $\begin{array}{c}\text { Conformable to curved surfaces of } \\
\text { arbitrary curvature. }\end{array}$ \\
\hline Thin & $<12.7 \mathrm{~mm}$ \\
\hline Stretchable & Strains of $>10 \%$ \\
\hline Soft & $<1 \mathrm{MPa}$ (linear modulus) \\
\hline Ultra low sensing range & $10-1 \mathrm{kPa}$ \\
\hline Wide sensing regime & Linear / proportional response \\
\hline Simple characteristics & Resolution $<5 \mathrm{~mm}$ (skin is $2.5 \mathrm{~mm}$ ) \\
\hline Pixel type sensing & $\begin{array}{c}\text { Existing techniques such as replica mold } \\
\text { by soft nanolithography }\end{array}$ \\
\hline Fabrication & \\
\hline
\end{tabular}

Three fundamental research questions are addressed in relation to the skin-level sensor developed in this thesis: 1) How does pillar geometry influence sensor sensitivity and the detectable pressure regime? 2) How can pillar structure be designed to optimize sensor performance? In other words, what are the modes of deformation and how does that influence the local electric field? And are there any secondary couplings relevant to the 
electromechanical response? and 3) What are the principles that play a dominant role in improving sensor behavior?

\subsection{Outline of thesis}

This chapter has introduced the topic of flexible pressure sensors and provided motivation for developing these devices based on cilia structures. In this thesis, a high sensitivity pressure sensor based on gold covered PDMS micropillar arrays is developed. Several designs are proposed, fabricated, and evaluated to optimize sensitivity and detectability in the low pressure range. The capacitance-type sensor is fabricated using soft nanolithography whereby the micropillars are individually electroded using a sputtering technique. Techniques for fabricating asymmetric easy to buckle pillar structures and a multi-level hierarchical design platform are developed. The resulting pressure sensor is stretchable, flexible, and has high sensitivity in the tactile sensing regime ( $<10 \mathrm{kPa})$ and excellent performance at detecting low pressures. Here, we present the highest reported sensitivity $\left[0.34 \mathrm{kPa}^{-1}\right]$ of a passive capacitance type sensor that is both flexible and stretchable. The results show that this sensor could be configured to detect very low pressures.

The influence of pillar design parameters on sensor performance is explored using experimental and computational simulation techniques. Finally, the microstructured sensor array naturally lends itself to the development of pixel-type pressure sensors and biomedical monitoring devices - the potential for both applications are investigated here. The following is a brief outline of the content of the thesis chapters.

Chapter 1. Introduction. This chapter presents the motivation, objectives, and background for the development of micropillar sensor arrays.

Chapter 2. Capacitive pressure sensing. This chapter outlines the fundamental concepts of electrostatics and capacitive sensing. 
Chapter 3. Fabrication of PDMS Micropillar Arrays. This chapter describes the fabrication process for the micropillar sensor arrays developed in this work.

Chapter 4. Sensor characterization. In this chapter, metrological characterization of the resulting pillar structure (designs) is carried out using standard imaging techniques. A detailed description of the experimental test setup and the sensor response of all fabricated designs is provided.

Chapter 5. Computational Analysis of Micropillar Sensor Arrays. Computational simulations were carried out to determine the deformation response of individual pillars and pillar arrays. A multiphysics platform is used to determine the coupled electromechanical response due to the applied pressure. In this way, 3D strain fields, stress fields, and electric fields is visualized for each design and the resultant capacitance change calculated. The results show that the simulations capture the essential mechanics of the problem as there is a good correlation between experimental and simulation results for the full-sized array. The simulations are used to determine the origins of improved sensitivity and correlate the dominant deformation modes with capacitance changes.

Chapter 6. Summary and Conclusions. This chapter presents a summary of the major thesis highlights and corresponding conclusions. In addition, it describes potential future directions that are natural offshoots of the work accomplished in this thesis. A summary of preliminary pre-seeded results is also described. 


\section{Chapter 2}

\section{Capacitive pressure sensing}

There has been a wave of research focused on developing pressure sensors for touch technology and electronic skins. A few of the main challenges that remain regarding this technology are: extreme precision and sensitivity of detecting micro-sized objects $(<<1$ $\mathrm{kPa}$ ), spatial resolution (1-2 mm), extended performance range on a single platform (1Pa $-10 \mathrm{kPa}$ ), and high sensitivity for both static and dynamic loads. The electromechanical principles of operation of pressure sensors developed to date are: i) capacitive, ii) resisitive (ohmic), iii) piezoresistive, and iv) piezoelectric. The advantages and disadvantages of the different techniques are outlined in the table below:

Table 2.2 Advantages and disadvantages of pressure sensors [60,63].

\begin{tabular}{|c|c|c|}
\hline $\begin{array}{c}\text { Sensor } \\
\text { Mechanism }\end{array}$ & Advantages & Disadvantages \\
\hline Capacitive & $\begin{array}{l}\text { Excellent sensitivity } \\
\text { Good spatial resolution } \\
\text { Large dynamic range }\end{array}$ & $\begin{array}{c}\text { Stray capacitance } \\
\text { Noise susceptible } \\
\text { Complexity of } \\
\text { measurement electronics }\end{array}$ \\
\hline Resistive & $\begin{array}{c}\text { High sensitivity, } \\
\text { Rapid transient response } \\
\text { A large working range } \\
\text { Small temperature dependence }\end{array}$ & $\begin{array}{l}\text { Hard to have spatial } \\
\text { resolution } \\
\text { Complexity of circuit }\end{array}$ \\
\hline Piezoresistive & $\begin{array}{l}\text { High spatial resolution } \\
\text { High scanning rate in mesh } \\
\text { Structured sensors }\end{array}$ & $\begin{array}{l}\text { Lower repeatability } \\
\text { Hysteresis } \\
\text { Higher power } \\
\text { consumption }\end{array}$ \\
\hline Piezoelectric & $\begin{array}{c}\text { High frequency response } \\
\text { High sensitivity } \\
\text { High dynamic range }\end{array}$ & $\begin{array}{l}\text { Poor spatial resolution } \\
\text { Dynamic sensing only }\end{array}$ \\
\hline
\end{tabular}


In this thesis, a capacitive technique is used to investigate sensor performance. The design is chosen such that the platform can be readily adapted to dielectric actuation without altering the basic design. PDMS is a flexible and stretchable material that is commonly used in soft nanofabrication technologies. Microstructured PDMS films have good elasticity (negligible hysteresis) over a range of strains and hence are an ideal candidate for electronic skins and devices that require mechanical flexibility and extensibility. In this thesis, it is shown how the design of the film microstructure can be tailored to improve sensor performance and enhance sensitivity. By careful selection of electrode type and dielectric polymer material, other sensor modalities and performance metrics can be attained. Spatially discrete sensing is achieved by either patterning the electrodes and or discretizing the micropillar array into sensor nodes connected by a stretchable conductive network. Figure 2.1 illustrates different transducer configurations for a patterned micropillar array. 


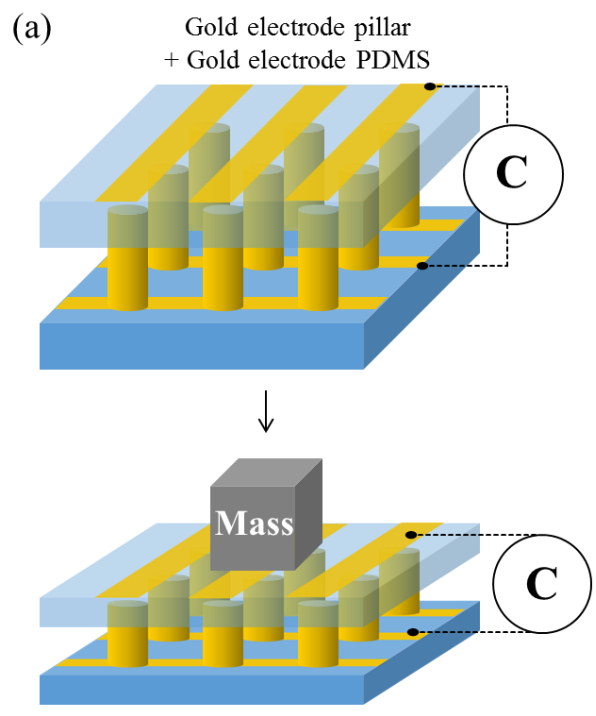

$<$ Capacitance sensing >
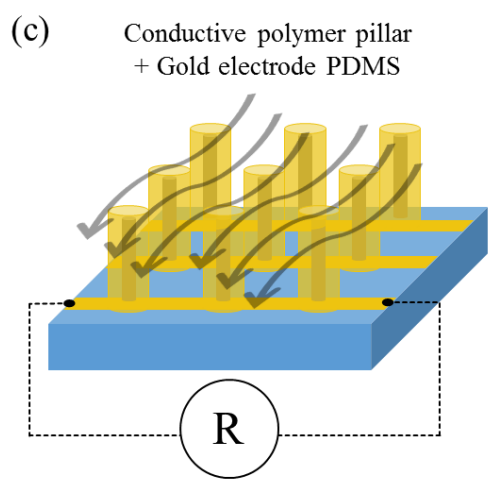

$<$ Resistive flow sensing $>$ (b)
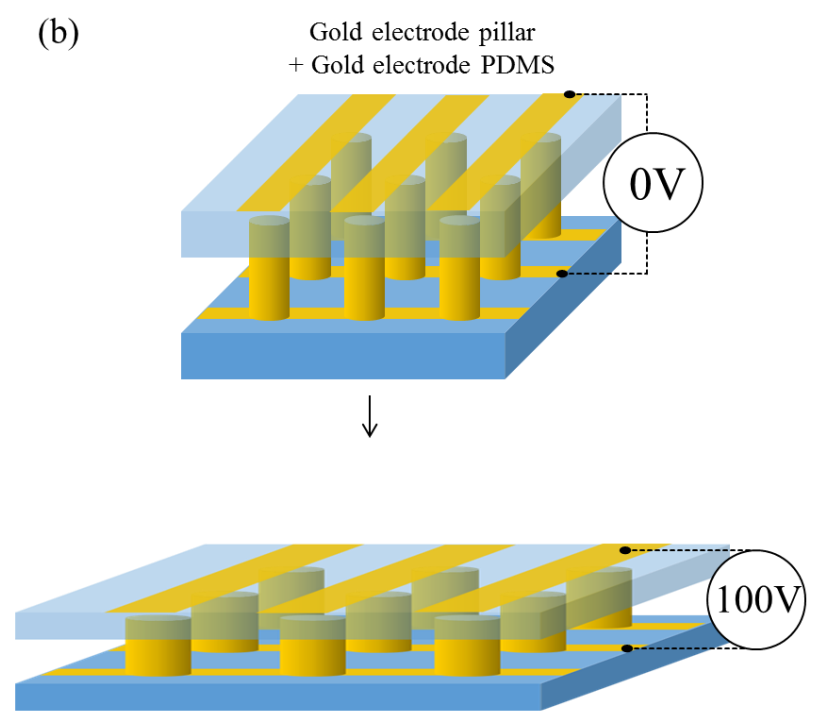

$<$ Actuation $>$

(d)

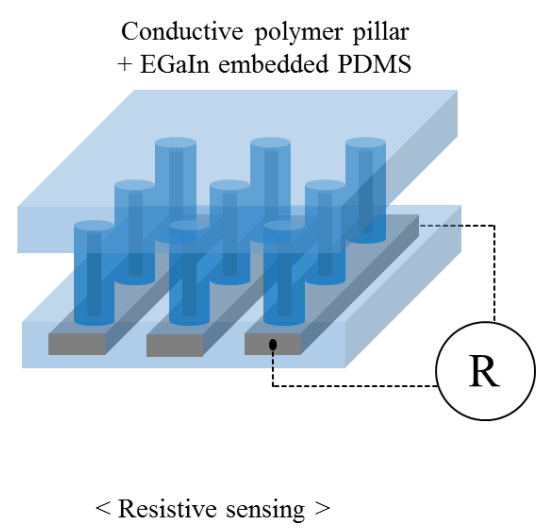

Figure 2.1 Micropillar array configuration and intended performance. a) closed micropillar array configured for capacitive pressure sensing (PDMS+ Au), b) closed micropillar array configured for actuation, c) open micropillar array for resistive flow sensing (conductive polymer $+\mathrm{Au}$ ), d) closed micropillar array configured for resistive sensing (conducting polymer + EGaIn).

\subsection{Capacitance-type sensors}

The pressure sensor is composed of an electro-deposited gold ( $\mathrm{Au}$ ) layer on a PDMS micropillar array and a metallic ( $\mathrm{Au})$ monolithic PDMS film. Connecting copper tape electrical leads to the measurement device completes the sensor circuit. Measurements can be taken in either open circuit or closed circuit configurations though the former is more common for sensor setups. Capacitive pressure sensors based on microstructured 
PDMS have been investigated before [Schwartz, Tee, Mei 2013, Nature Communications] with reported sensitivities up to $8.3 \mathrm{kPa}^{-1}$. The main drawback of these sensors is the change in the local dielectric constant, which can occur due to impurities that contaminate the electric field domain.
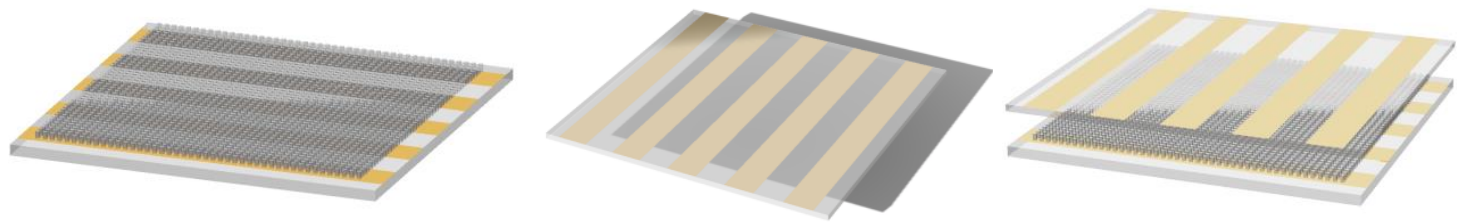

Figure 2.2 (left) PDMS micropillar array, (center) electroded monolithic film, and (right) fully assembled pixel-type sensor.

\subsubsection{Elementary laws of capacitors}

An ideal parallel plate capacitor typically consists of two conducting rigid plates separated by a dielectric medium. The behavior can be understood by considering the elementary response of two dissimilar but equal charges brought into close proximity of each other. The Coulombic interaction describes the action at a distance electrical force of pull felt because of the affinity of the charges to come together. Opposite behavior arises when identical charges are brought in close proximity. The field lines between the charges outlines the action at a distance that would be felt on a test charge placed anywhere in the domain.

\subsubsection{Capacitance change in an Electric field}
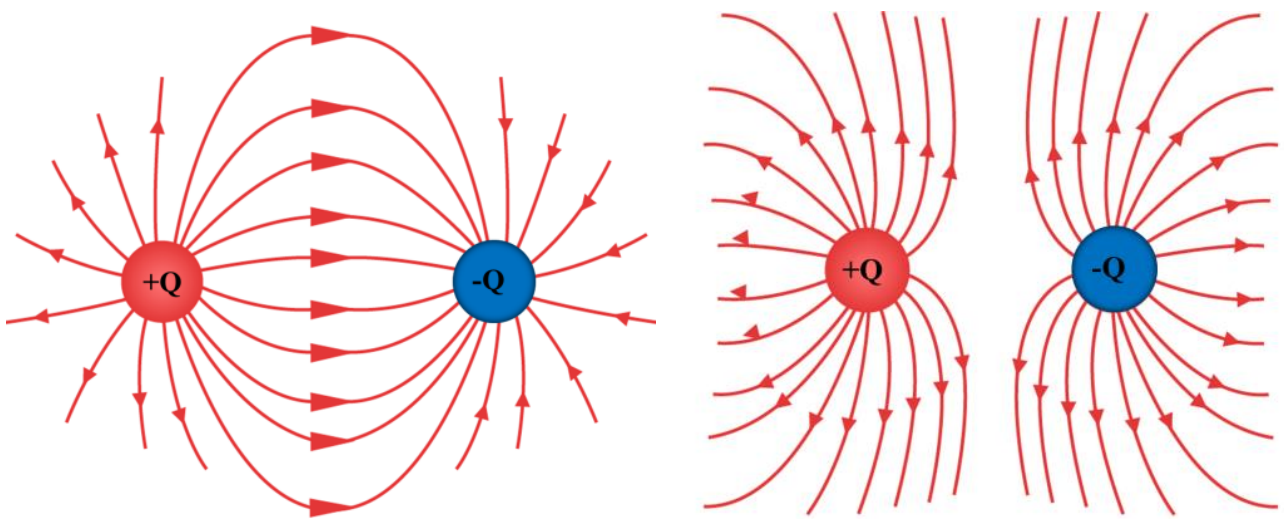

Figure 2.3 Dissimilar charges in close proximity and similar charges in close proximity illustrating Coulomb's Law. 
Mathematically, Coulomb's law is

$$
F=k_{e} \frac{\left|q_{1} q_{2}\right|}{r^{2}}
$$

where $\mathrm{F}$ is force interacting between static electrically charged particles, $\mathrm{k}_{\mathrm{e}}$ is Coulomb's constant $\left(\mathrm{k}_{\mathrm{e}}=8.99 \times 10^{9} \mathrm{~N} \mathrm{~m}^{2} \mathrm{C}^{-2}\right), \mathrm{q}_{1}$ and $\mathrm{q}_{2}$ are the magnitudes of the charges, and the scalar $\mathrm{r}$ is the distance between the charges.

The parallel plate capacitor is the simplest capacitor configuration and most relevant for the geometry considered in this thesis. When a potential difference is applied across the electrodes, this results in an electric field, which is typically uniform between the plates. The electric field also extends beyond the plates into the surrounding medium. At the edges of the plate as well as in the immediate vicinity there exists a domain with a nonuniform field called the fringe field (Figure 2.4). In the MEMS field, fringe effects have been successfully employed in several applications as part of the sensing mechanism of rigid microcapacitors.

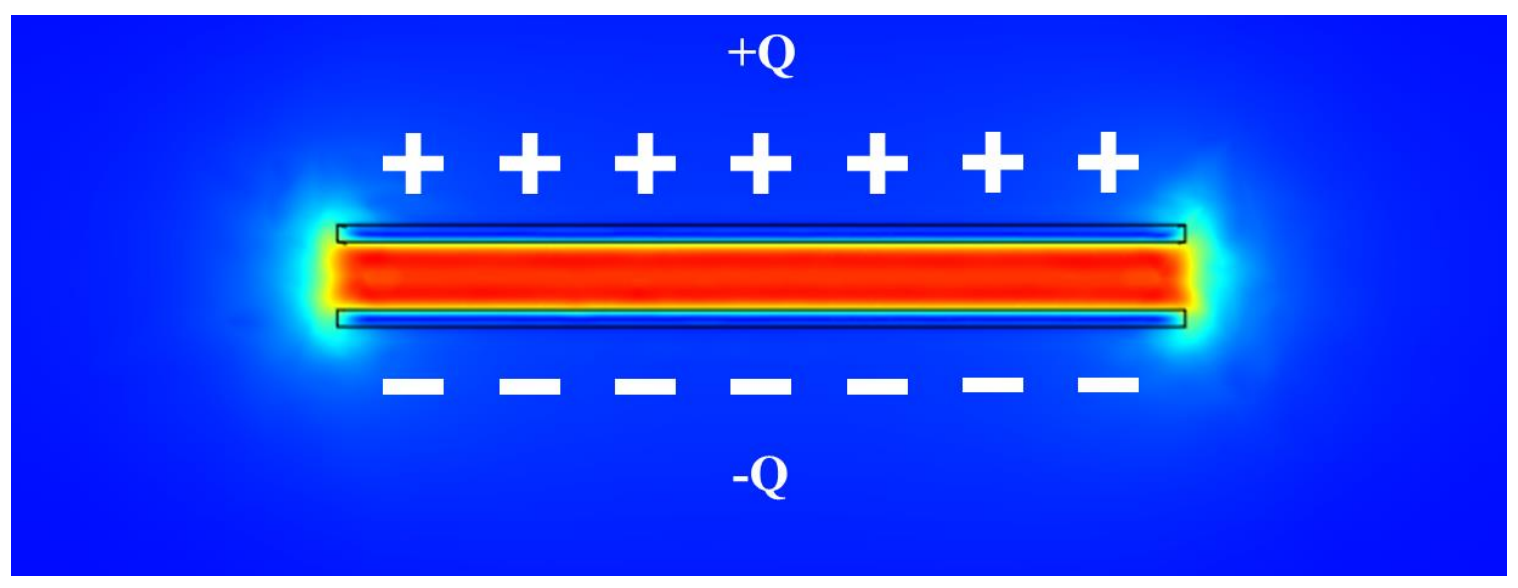

Figure 2.4 A simple parallel plate capacitor consisting of a monolithic dielectric and rigid monolithic electrodes and the resulting electric field domain.

Microstructured PDMS sensors create air gaps between the electrodes. This modifies the electric field due to the variance in dielectric constant between the pillars. The resulting electric field is shown in Figure 2.5. The air gaps also contribute to the reduced relaxation 
timescales [9]. The capacitance of an ideal parallel plate capacitor reflects the ability of the unit to store charge. This quantity is a function of the area of the electrodes, the distance between the electrodes, and the dielectric constant of the filler material. With a microstructured dielectric, the array is essentially a series of capacitors arranged in parallel connected by the resistance of the continuous elements. The capacitance changes as a function of the applied mechanical loading because of the deformation of the dielectric layer i.e. narrowing of the gap between electrodes.
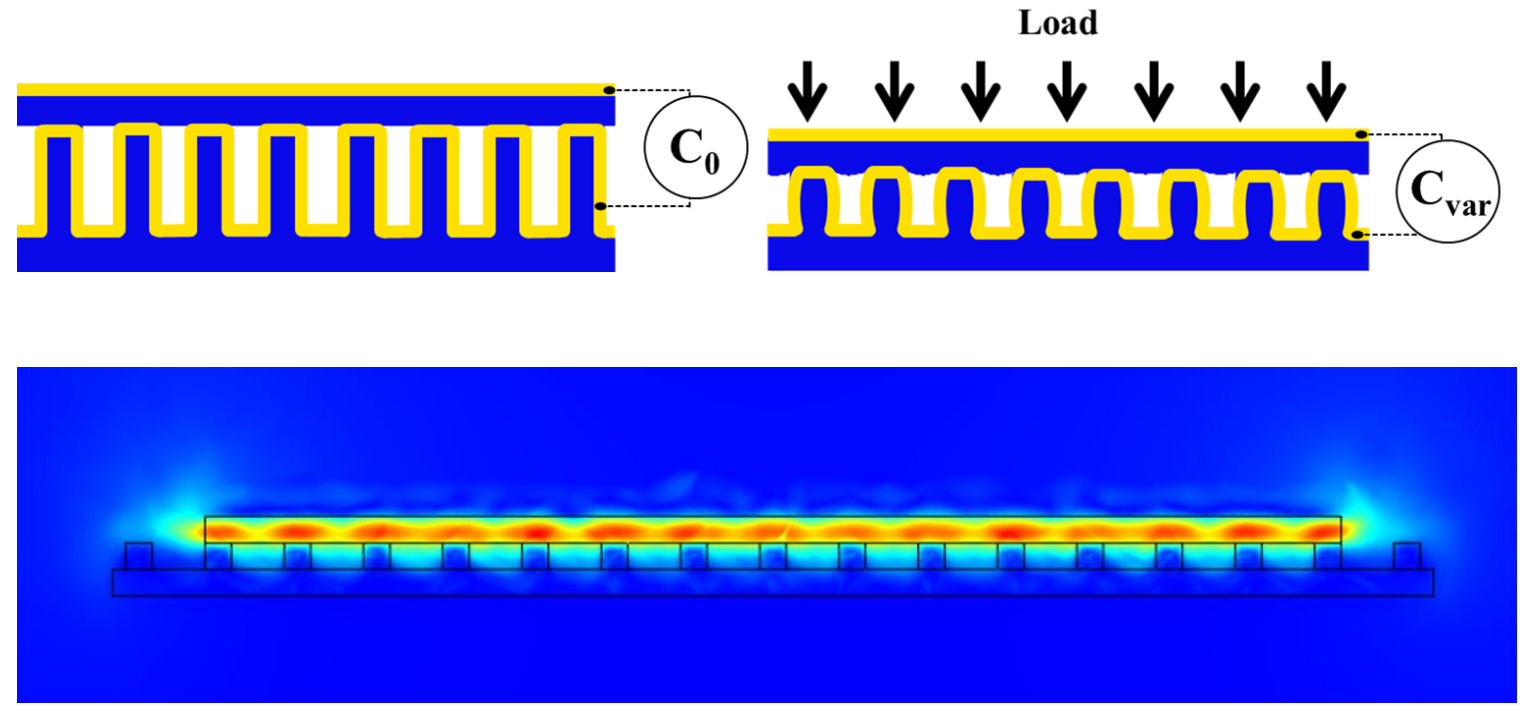

Figure 2.5 Top: Basic principle of capacitive sensors due to change in the distance between the electrodes, d. left) undeformed micropillar sensor and right) deformed micropillar sensor. Bottom: Electric field of micropillar array.

The capacitance for a single unit i.e. parallel plate capacitor considering rigid parallel plate electrodes is outlined here. Consider 2 parallel and flat overlapping rigid electrodes of area A, separated by a distance $\mathrm{d}$. In the uncharged state, there is zero charge on the electrodes (Figure 2.6). A potential difference $\Delta \mathrm{V}$ is created between the two electrodes by moving charge from one plate unto the other. The relative permittivity of air is $\varepsilon_{0}$ and the relative permittivity of the inserted dielectric material is $\varepsilon_{r}$. 


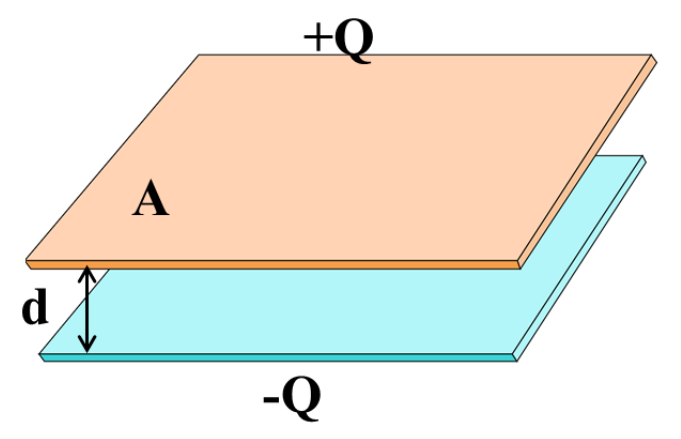

Figure 2.6 A simple parallel plate capacitor.

The relationship between the total charge and the electric potential difference is

$$
\mathrm{Q}=\mathrm{C} \times \Delta \mathrm{V}
$$

where $\mathrm{C}$ is the proportionality constant dependent on size and shape and type of material. Typical capacitance values are in the $\mathrm{pF}$ to $\mathrm{mF}$ range. Neglecting the edge and fringe fields shown in Figure 2.4 and focusing on the uniform field contained between the electrodes is equivalent to assuming infinitely large charged plates with planar symmetry. The surface charge density of either plate is $\sigma$. Gauss' law states that the electric flux through an enclosed surface (A') is equal to the charge enclosed qenc (2.3). Thus, the contained electric field between the plates is given by (2.4). The potential difference in calculated along the electric field line path from the positive plate to the negative plate as in $(2.5)$.

$$
\begin{gathered}
\oiint_{S} \overrightarrow{\mathbf{E}} \times d \overrightarrow{\mathbf{A}}=\frac{q_{\text {Ene }}}{s_{0}}, \\
E A^{\prime}=\frac{q_{\text {Enc }}}{s_{0}}=\frac{\sigma A^{I}}{s_{0}} \Rightarrow \frac{\sigma A^{J}}{s_{0}},
\end{gathered}
$$




$$
\Delta V=V_{-}-V_{+}=-\int_{+}^{-} \overrightarrow{\mathbf{E}} \times d \overrightarrow{\mathbf{s}}=-E d
$$

The capacitance is related to the magnitude of the potential difference as noted earlier in (2.2). From that definition, we have

$$
C=\frac{Q}{|\Delta V|}=\frac{\varepsilon_{0} A}{d}
$$

For a dielectric other than air, (2.6) becomes

$$
C=\frac{Q}{|\Delta V|}=\frac{\varepsilon_{0} \varepsilon_{r} A}{d}
$$

Therefore, the capacitance increases proportionally with the electrode area for a given potential difference. It is also inversely proportional to the distance between electrodes and directly proportional to the dielectric selected. Hence, any changes in these quantities will cause a change in the capacitance measurement of a sensor device whereby the background capacitance is screened out. The principle of capacitive pressure sensing hence hinges upon the design of a device that reliably and repeatedly deforms in a deterministic way due to applied pressure. The change in the gap distance is the most obvious control parameter.

It should be noted that the environmental capacitance and unintended contamination of the locale can cause parasitic effects and hence false sensor readings. It is therefore important in sensor design to try to minimize the magnitude of these factors in relation to the strength of the actual sensor reading. In this regard, some sensor designs rely on additional circuitry, and or a secondary detection mechanism, or pursue entirely alternative measurement schemes as highlighted earlier. 


\subsection{Capacitance in a nonuniform electric field}

The micropillar array described in this thesis shows improved sensitivity in comparison to other flexible and stretchable sensors. An in depth discussion is given in the results section of Chapter 4 and the computational analysis of Chapter 5. Here, we present the background for the electrical part of the improved performance. The primary effect that causes a change in capacitance is the change in the distance between electrodes due to deformation of the PDMS monolithic layer and the pillar. The micropillar array is a bit of a complicated design electrostatically because it has a nonuniform electric field (in contrast to the simple picture in Figure 2.4). This situation arises because the bottom electrode is neither flat nor planar (the pillars are electroded) making the problem difficult to solve analytically (see Figure 2.7). For complex nonuniform fields, as is the current case, computational tools are ideal to quantify and visualize the electromechanical response. Figure 2.7 shows a close up view of the simulated electric field in a single pillar, where the top electrode is set to $1 \mathrm{~V}$ and the bottom electrode (which runs in the gap and along the vertical sides and over top of the pillar) is set to ground. The electric field is overlaid on the deformed configuration, which is outlined in red. The simulation was conducted in the multiphysics finite element software COMSOL. The capacitance is calculated using (2.2), (2.3) and (2.5), which involve spatial variations (3D) in the dielectric and electrode surface geometries.

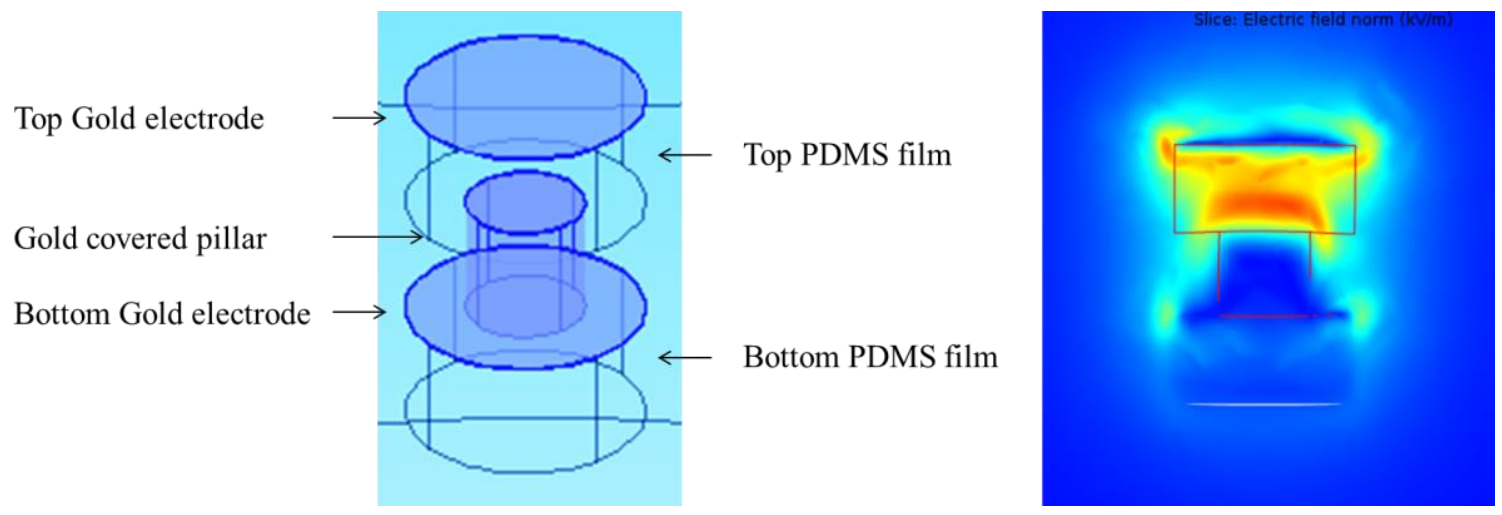

Figure 2.7 Electric field simulation for a single pillar. Left) shaded electrode regions of a single pillar and right) The bulk of the field is contained in the monolithic film and gently extends into the pillar region and gap. Note the fringe fields at the edges of the PDMS film and on top of the Au electrode. 


\subsubsection{Secondary mechanisms}

Two secondary mechanisms responsible for increased sensor sensitivity are: i) fringe field interference and ii) contained field alteration. The placement of the object in near proximity of the sensor interferes with the fringe field and hence changes the capacitance of the system (Figure 2.8). This has proven to be a reasonably reliable technique for proximity sensing and detecting very small masses (molecules) [63]. Figure 2.8 illustrates how the approach of an object (conducting or insulating) affects the fringe field of a capacitive sensor. The obvious drawback here is that the mechanism is better suited for on/off applications since it detects presence of an object through the locally disturbed fringe field, which is then difficult to decouple from the capacitance change due to the actual pressure loading.

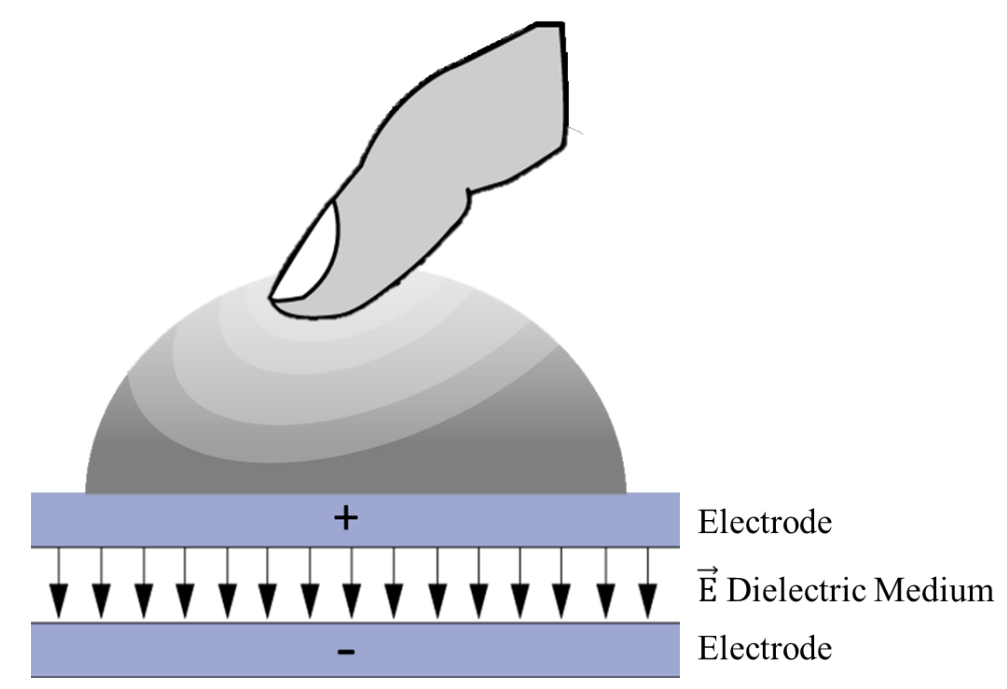

Figure 2.8 Object interferes with the fringe field and changes the capacitance of the system (proximity sensing).

The capacitance of the device is influenced by local and nonuniform deformations of the monolithic film and pillar that alter the local electric field by causing a spatial variation in the permittivity layer between the Au electrodes. Figure 2.9 shows the variation in the electric field as the mechanical contact occurs between a massless object and the micropillar sensor. The simulated results clearly show that the bulk of the electric field is 
contained in the top monolithic PDMS film. A portion of the field extends along a fraction of the pillar length.
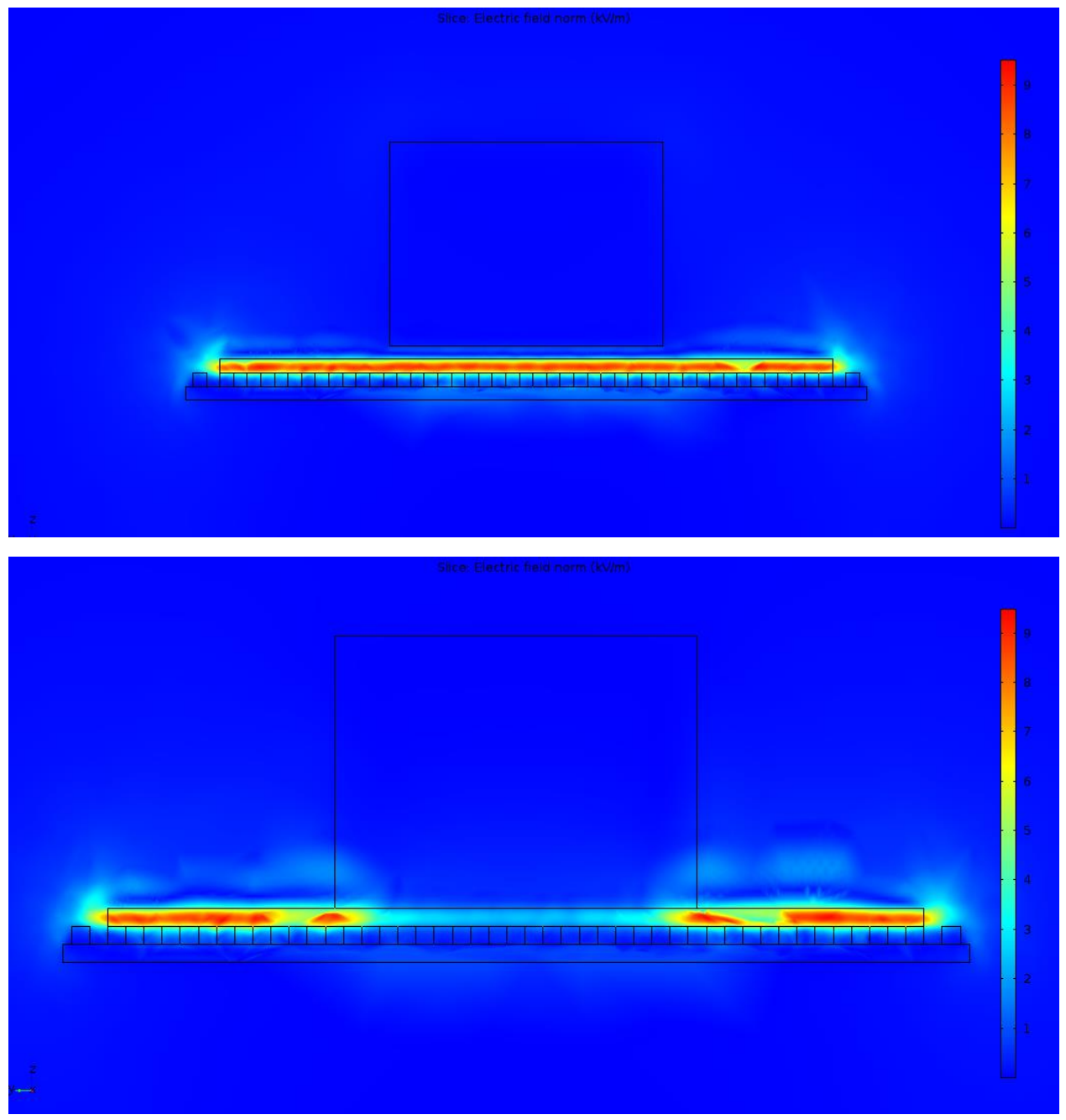

Figure 2.9 Changes in the electric field due to object placement.

The concepts outlined here are revisited in the extensive results provided in Chapter 5 where all the pillar designs are considered. The capacitance is calculated for the different sensor array geometries and the deformation, stress, and electric fields are presented and analyzed. 


\section{Chapter 3}

\section{Fabrication of PDMS Micropillar Arrays}

\subsection{Introduction}

\subsubsection{Overview of materials and process selection}

In order to achieve compliant microstructures that easily respond to the outer environment, material selection is important. Elastomers are a top candidate easily amenable to existing fabrication techniques such as soft lithography. The most common material used in this technique is polydimethylsiloxane (PDMS), a soft low cost elastomer that is easy to mold, has low hysteresis at high strains, and mechanically durable. It can be stretched over 100 times its original length without damage making it an excellent choice for flexible and stretchable systems [64]. The PDMS is used as a base material and flexible substrate for soft electronics [65]. Since it is electrically insulating, it is also employed as a dielectric, where the dielectric constant is 2.7 [66].

Flexible and stretchable electrodes are required to render the entire device deformable. The electrodes can be directly patterned on the PDMS layer and have stable and high electrical conductivity. Several studies have shown that sputtered gold on PDMS serves as an excellent conducting layer [67], and the process is easy. Sputtered gold has a conductivity of $4.10 \times 10^{7} \mathrm{~S} / \mathrm{m}$ and is environmentally stable, with excellent 
resistance to oxidation and corrosion [68]. Micro patterning of a thin layer of gold at the micro- or nano- scale can be performed with a general physical vapor deposition technique and employing a shadow masking to pattern as needed. The drawbacks of sputtered gold is the comparatively higher cost compared to other electrodes such as carbon grease and its brittle nature as it is susceptible to microcracking - though that does not compromise its electrical performance much. Furthermore, it possible that the thinly deposited Au layer (30nm) can be damaged or exfoliated by mechanical force or repeated load [77]. Over certain load and or strain thresholds, peeling occurs in addition to microcracks and the electrical connectivity can deteriorate. Begley et al. showed uncompromised actuation of dielectric elastomers fabricated with sputtered metallic electrodes undergoing large deformations [69]. Applying a thin stiff layer to a prestretched soft substrate results in buckling of the stiff layer when the prestretch is released. Using this technique with sputtered gold electrodes results in sustained conductivity up to a stretch of $50 \%$ as reported in the literature 78 for $50 \%$ value). The parameters that control the buckled or wrinkled pattern and hence finite extensibility are relative layer thicknesses, relative layer stiffnesses, and substrate and nature of prestretch (uniaxial versus biaxial etc.) [70]. Figure 3.1 shows the fabrication process, SEM image of the winkling pattern of the Au electrode surface, and change in resistivity with stretch. Electrical conductivity is acceptable up to $\sim 20 \%$ strain in this example. 


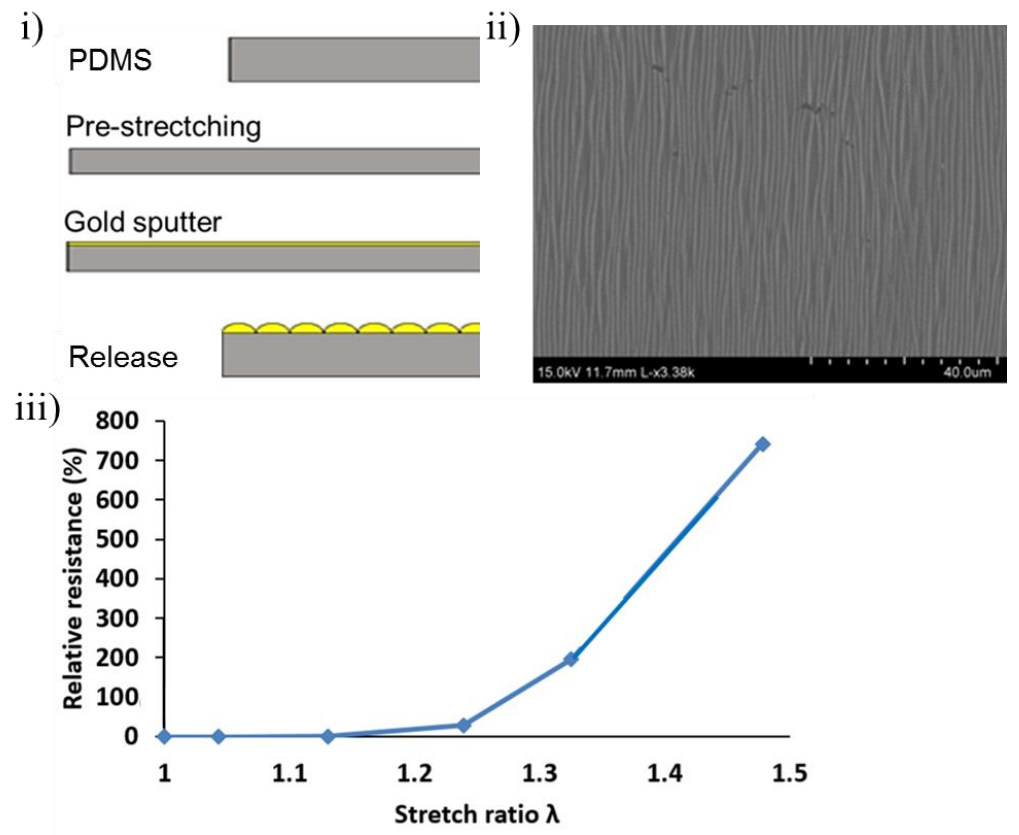

Figure 3.1 i) Fabrication of Au buckled electrodes. ii) Micrograph of buckled Au electrode. iii) Relative resistance versus uniaxial stretch for buckled Au electrode film.

\subsection{Fabrication approach}

In this thesis, micropillar arrays are fabricated using a soft nanolithography technique [71]. Soft lithography is a technique used to create microstructures by means of replica molding and embossing an elastomer on a soft mold created from a hard silicon (SI) mold. The SI mold can be used up to 30 times if the mold is deposited with the release agent (trichloro(3,3,3-trifluoropropyl)silane). Most microfluidic systems used for bio sensing or chemical detection are fabricated with this technique [72]. Figure 3.2 and 3.3 and Table 3.1 show a schematic of the fabrication steps and specifications of the resulting samples, respectively. The bottom layer is the PDMS micropillar array. Gold electrodes are deposited over the individual pillars and between the pillar gaps in a parallel line pattern using a shadow mask $25 \mathrm{~mm}$ x 50mm. A top monolithic PDMS film 
is placed on top of the Au micropillar array and is subsequently electroded (lines are orthogonal to the bottom Au layer) using a shadow mask. The electroded lines form a grid pattern to create through thickness capacitive cells or pixels. The SI wafer mold was fabricated using photolithography with dry reactive ion etching (DRIE) [73].

(a)

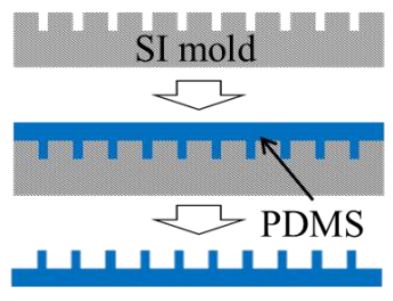

(b)

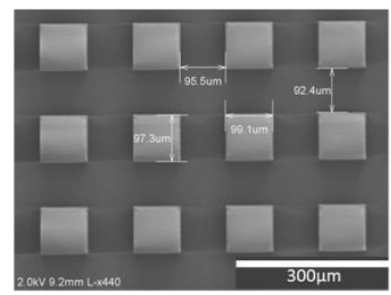

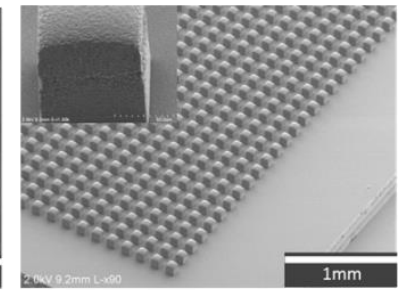

Figure 3.2 (a) Schematic of the PDMS molding process by replica modling. (b) SEM images of the micropillar array.

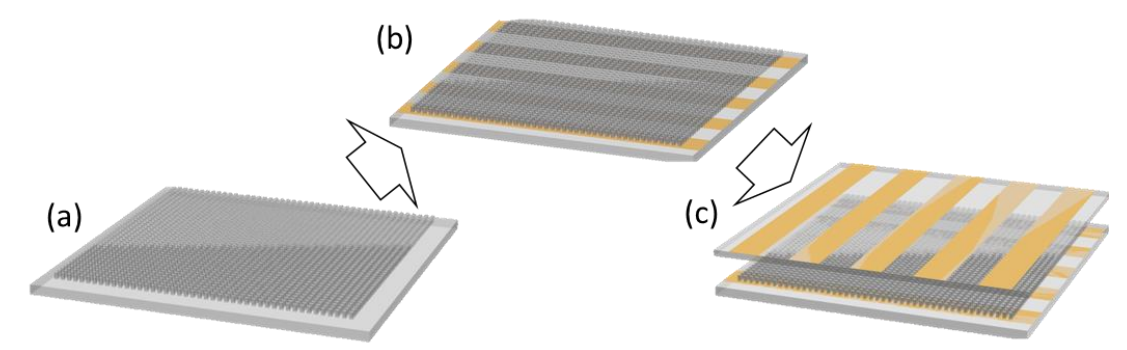

Figure 3.3 Schematic of electroding process and sensor assembly. (a) PDMS micropillar array layer (b) Au electroded pillar surface patterened into lines using a shadow mask $25 \mathrm{~mm}$ x $50 \mathrm{~mm}$ (c) A top monolithic PDMS film placed on top of the Au micropillar array and is subsequently electroded using a shadow mask. The electroded lines form a grid pattern to create through thickness capacitive cells or pixels. 
Table 3.3 Summary of sample dimensions fabricated.

\begin{tabular}{|c|c|c|c|c|c|c|}
\hline \multirow{2}{*}{ Sample \# } & \multirow{2}{*}{$\begin{array}{c}\text { Cross } \\
\text { section } \\
\text { shape } \\
\end{array}$} & \multirow{2}{*}{$\begin{array}{c}\text { Aspect } \\
\text { ratio }\end{array}$} & \multicolumn{3}{|c|}{ Dimension $(\mu \mathrm{m})$} & \multirow{2}{*}{$\begin{array}{l}\text { Film } \\
\text { thickness } \\
(\mu \mathrm{m})\end{array}$} \\
\hline & & & gap & height & width & \\
\hline 1 & \multirow{9}{*}{ Square } & $1: 1$ & 100 & 100 & 100 & \multirow{14}{*}{200} \\
\hline 2 & & $1: 2$ & 100 & 200 & 100 & \\
\hline 3 & & $1: 3$ & 100 & 300 & 100 & \\
\hline 4 & & $1: 1$ & 200 & 100 & 200 & \\
\hline 5 & & $1: 3$ & 35 & 105 & 35 & \\
\hline 6 & & $1: 5$ & 21 & 105 & 21 & \\
\hline 7 & & $1: 7$ & 15 & 105 & 15 & \\
\hline 8 & & $1: 6$ & 17.5 & 105 & 17.5 & \\
\hline 9 & & $1: 9$ & 11.7 & 105 & 11.7 & \\
\hline \multirow{3}{*}{10} & \multirow{3}{*}{ Hybrid } & $1: 3$ & 145 & 100 & 33.3 & \\
\hline & & $1: 5$ & 65 & 80 & 16 & \\
\hline & & $1: 7$ & 14 & 60 & 8.6 & \\
\hline 11 & Triangle & $1: 1$ & 48 & 100 & 152 & \\
\hline 12 & Circle & $1: 1$ & 87.2 & 100 & 112.8 & \\
\hline
\end{tabular}

\begin{tabular}{|c|c|c|c|c|c|c|}
\hline Sample \# & $\begin{array}{c}\text { Cross } \\
\text { section } \\
\text { shape }\end{array}$ & OR:IR & IR & OR & gap & height \\
\hline 13 & \multirow{6}{*}{$\begin{array}{c}\text { Half } \\
\text { Circle } \\
\text { (Easy } \\
\text { to } \\
\text { buckle) }\end{array}$} & $5: 3$ & 42.3 & 70.5 & 100 & 100 \\
\hline 14 & & $5: 4$ & 75.2 & 94.0 & 100 & 100 \\
\hline 15 & & $5: 3$ & 42.3 & 70.5 & 200 & 100 \\
\hline 16 & & $5: 4$ & 75.2 & 94.0 & 200 & 100 \\
\hline 17 & & $5: 3$ & 42.3 & 70.5 & 300 & 100 \\
\hline 18 & & $5: 4$ & 75.2 & 94.0 & 300 & 100 \\
\hline
\end{tabular}

\subsubsection{PDMS micropatterning techniques}

With a soft lithography technique, the mold can be patterned with a resolution on the microscale $(\sim 100$ microns) or nanoscale $(\sim 100 \mathrm{~nm})$ [73]. There are two well-known approaches for molding PDMS; one is direct molding with the Si wafer mold and the second is molding with the material SU-8 [74]. For this study, the direct molding method 
is used. The array pattern is designed using the mask making CAD software, L-edit. A chromium (Cr) mask is designed and fabricated with a Heidelberg $\mu$ PG 501 Mask Maker system. With the direct write approach, $\mathrm{Cr}$ is used to absorb UV(Ultraviolet) rays from the machine and transfer the pattern. The Cr mask is then used for patterning the photoresist on a Si wafer in the subsequent UV exposure steps.

\subsubsection{Deep Reactive Ion Etching (DRIE)}

The Si wafer is deposited with a $3 \mu \mathrm{m}$ photoresist (MEGAPOSIT TM SPR TM 220) and soft baked at $115^{\circ} \mathrm{C}$ for 90 seconds. A Cr mask is aligned with the wafer and then exposed to UV light $\left(20 \mathrm{~mW} / \mathrm{cm}^{2}\right)$ for 9 seconds. After a post exposure bake, the wafer is developed with AZ 726 MIF developer (AZ Electronic Materials USA Corp) for 30 seconds. The patterned Si wafer is then etched with a SPTS Pegasus 4 DRIE tool. To improve the texture of the sidewall surfaces, descum residual Photoresist is applied to the Si wafer with YES-CV200RFS Plasma stripper. A 10:1 mixture of PDMS elastomer (Sylgard 184, Dow Corning) to cross-linker is combined and mixed well. The PDMS solution with mass of $70 \mathrm{~g}$ is placed on the SI wafer and spin-coated for 30 seconds at $300 \mathrm{rpm}$. The polymer solution is degassed for 10 minutes in a vacuum chamber and cured at $100^{\circ} \mathrm{C}$ for $1 \mathrm{hr}$. The microstructured surface is then carefully detached from the mold very slowly. For high aspect ratio pillars, there can be difficulties in extracting the array from the mold due to strong surface energies, which are a function of contact length. In other words, the PDMS can stick to the inside of the Si mold resulting in broken pillars and a failed extraction[75]. Therefore, trichloro(3,3,3-trifluoropropyl)silane (Sigma Aldrich) was vacuum deposited to reduce the surface free energy and facilitate release from the mold [72]. 
A hierarchical pillar array is proposed to improve sensor performance. The design has multiple contact levels and integrates three different aspect ratios (AR) and three different gap distances on a single platform. It is desired to fabricate this unique combination of different pattern levels on single wafer mold. This means that additional mask layers and fabrication steps are needed. The tailored fabrication process was successfully developed in this thesis. Specifically, the photoresist (PR) is used as the first masking material, and the SI oxide layer and metal ( $\mathrm{Cr}$ or $\mathrm{Al})$ is used as the second and third masking materials on the bare Si surface. The materials form a uniform thin layer on the Si wafer and don't react with any other materials in the process [76]. The modified fabrication process is shown in Figure 3.5 below. First, a Si oxide layer is formed on the bare Si wafer surface by an oxide atomic layer deposition technique. The layer is patterned with the PR layer and exposed to have the first open area. Next, the $\mathrm{Cr}$ layer is deposited by a sputtering technique. The Cr layer can be selectively etched by a highly acid chromium etchant. This creates patterns on the oxide layer and forms a second open area. The last masking layer can be made with PR and the entire surface exposed to UV light to be etched. 

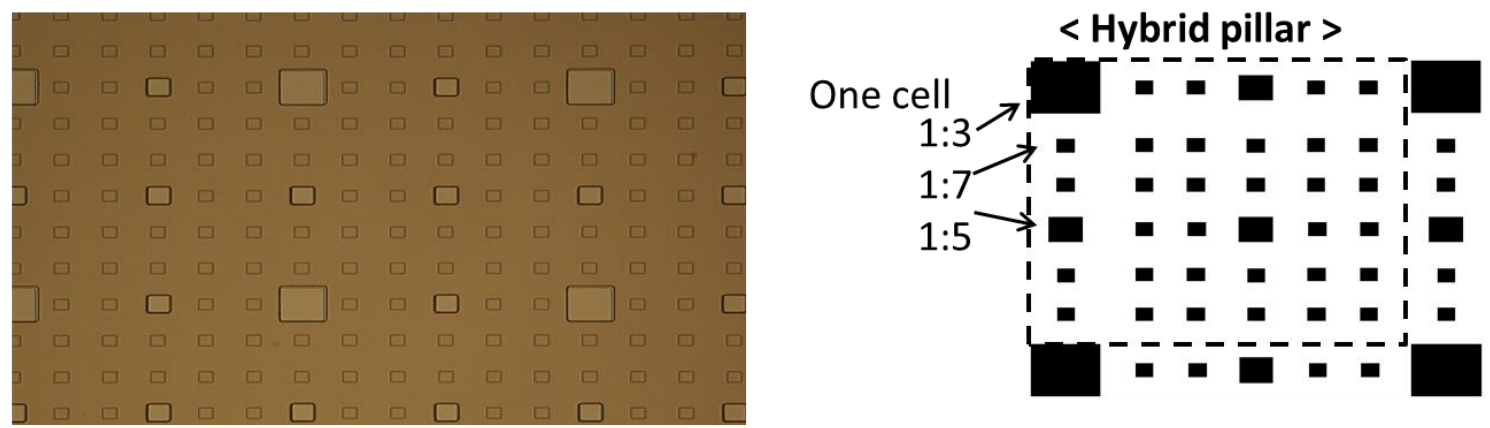

Figure 3.4 Hybrid mask design is a combination of AR 1:3,1:5,1:7 pillars on a single platform. left) SEM image of pattened SI wafer. right) Schematic of hybrid design.

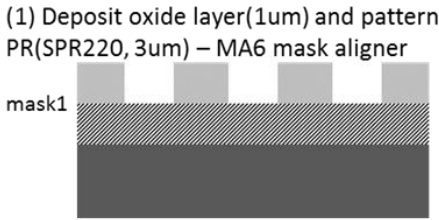

(2) Strip PR - Yes plasma stripper Etch Si oxide - Lam or P5000

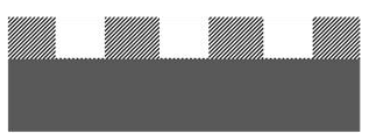

(3) Sputter $\mathrm{Cr}$ or Al (0.1um)

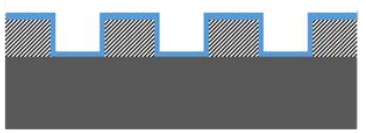

(4) Align and pattern PR mask2

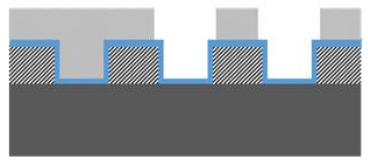

(5) Wet etch $\mathrm{Cr}$ or $\mathrm{Al}-$ Acid bench 12 , *need to take more time than etch rate

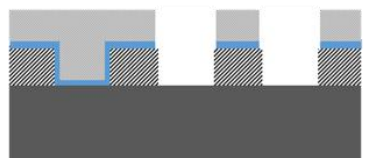

(6) Strip PR and pattern PR

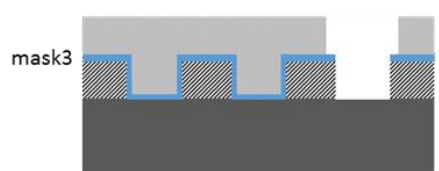

(7) Etch 30um $1^{\text {st }}$ Si layer

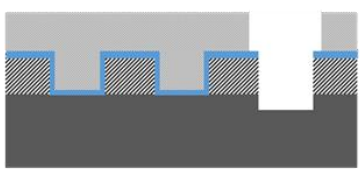

(8) Strip PR and etch 30um $2^{\text {nd }}$ Si layer

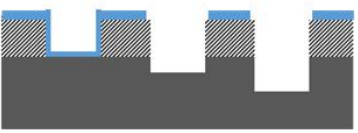

(9) Wet etch $\mathrm{Cr}$

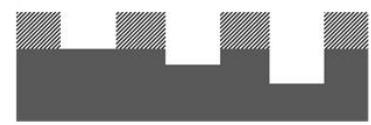

(10) Ignore Si oxide layer and etch 40um $3^{\text {rd }}$ Si layer

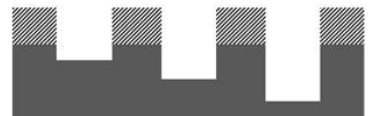

Figure 3.5 Fabrication steps for hybrid micropillar mold. 


\subsection{Fabrication results}

As can be seen from the SEM images in Figure 3.6, the pillar surfaces are smooth and regular and the array was uniform. The pillar structures had a pitch fidelity within \pm $5 \%$.
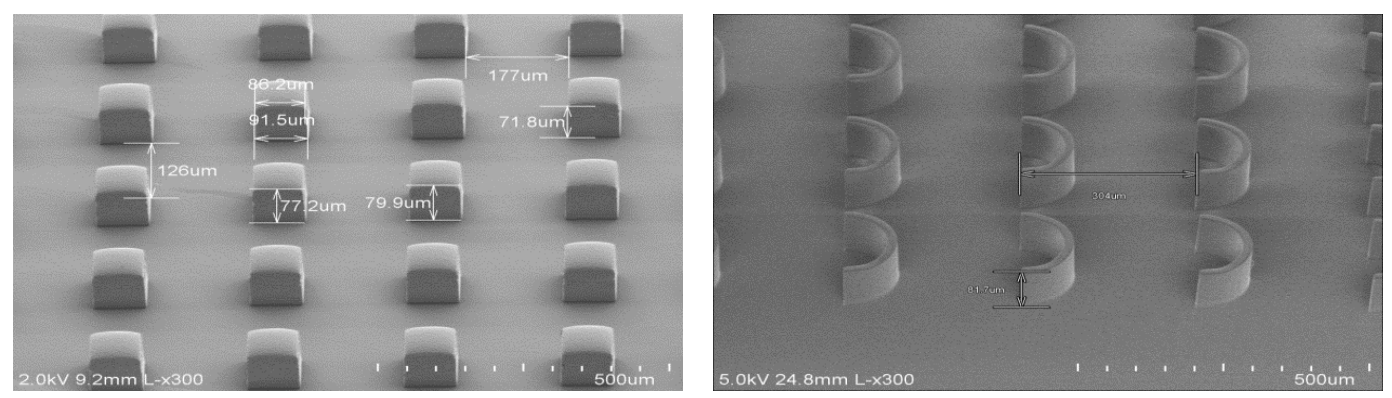

Figure 3.6 SEM images of as fabricated micropillar array surfaces a) $200 \mu \mathrm{m}$ gap 1:1 aspect ratio b) $200 \mu \mathrm{m}$ gap half circle pillar.

\subsubsection{Electroding technique}

To electrode either the PDMS micropillar array or the PDMS monolithic film, a shadow mask made of $0.07 \mathrm{~mm}$ thick Mylar® Polyester Film is applied to the surface in a pattern of five $20 \mathrm{~mm}$ by $20 \mathrm{~mm}$ rectangular strips. Au was deposited (30 nm) on the surface of the sample using a sputter-coating technique (Kurt J. Lesker, Lab 18) and following settings reported by University of Michgan LNF laboratory [79]. It was confirmed that the Au was uniformly deposited along the vertical walls of the pillars by SEM images. Figure 3.7 shows a zoomed in view of electroded pillars. 'LNF Lab 18 Standard Films Characterization'[80] also showed that sputtered gold film thickness measurement results with Dektak 6M Film thickness metrology equipment. The assembly is flexible and if buckled Au electrodes are used, inextensible. 

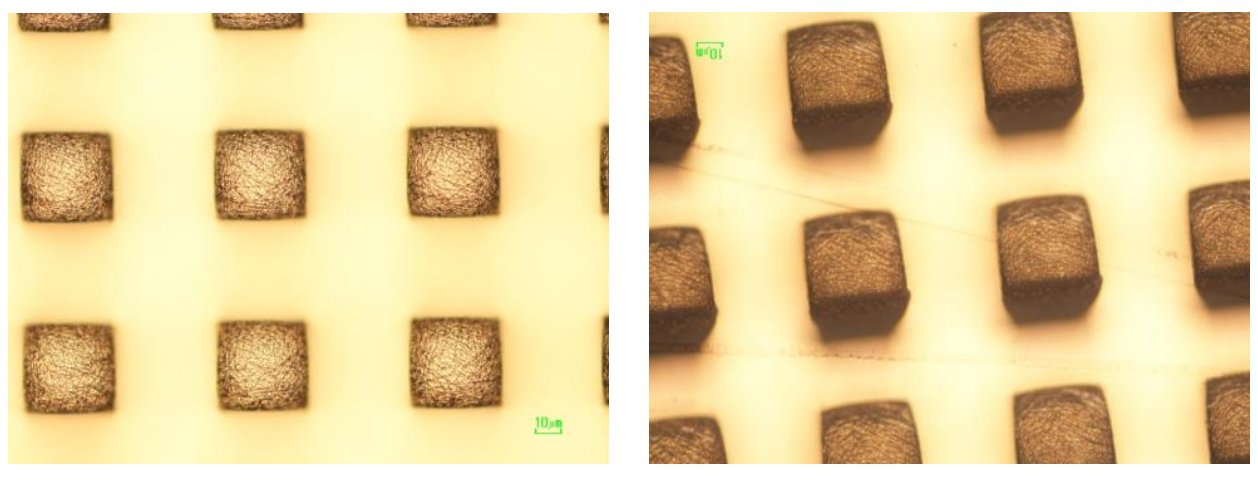

Figure 3.7 Individual pillars are uniformly coated with Au forming a thin nanoscale layer along the vertical walls, pillar top surfaces, and in the gaps between the pillars.
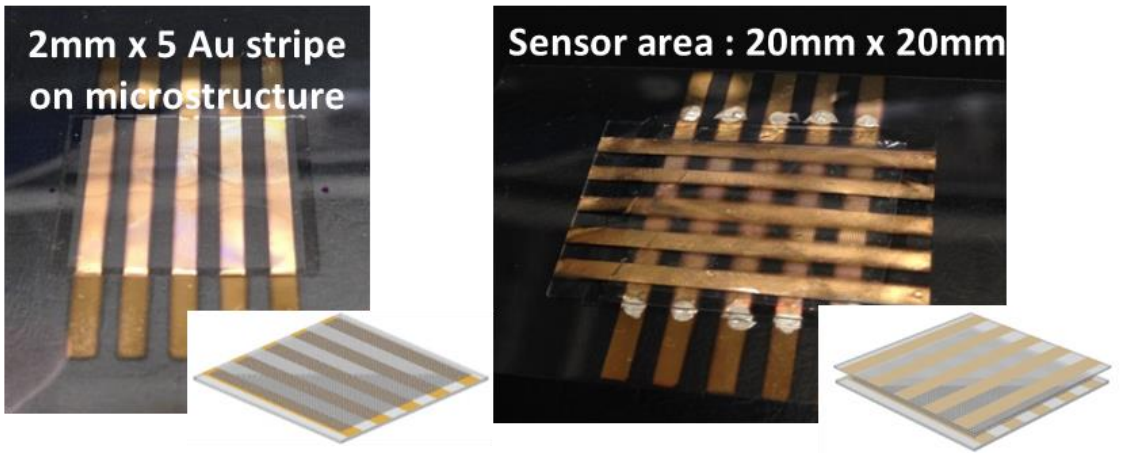

Figure 3.8 Au electroded micropillar array and final assembly for capacitive pixel sensing.

Using the aformentioned fabrication and electroding techniques, micropillarbased sensors of varying designs are fabricated. The design parameters include gap distance, aspect ratio, cross section shape, and size of pillars. These structural features influence the deformation mechanisms of the pillars and the overall sensor characteristics. Characteristics of the micropillar arrays fabricated in this work and sensor performance of the assembled sensors are described in Chapter 4. The correlation between pillar geometry and electromechanical coupling in the sensor device is further probed in Chapter 5 using a computational approach. 


\section{Chapter 4}

\section{Sensor characterization}

\subsection{Micropillar design}

In a typical pressure sensor, micropillar arrays are fabricated using a conventional soft photolithography technique as described in Chapter 3. Eighteen different pillar patterns were fabricated and tested for sensor performance. The primary objectives were to assess the basic device characteristics and investigate the influence of pillar design on the electromechanical coupling and capacitance output. Primary metrics of interest are sensor sensitivity and sensing range. The devices use an identical material platform so all the fabricated sensors are stretchable and flexible. The design variables are pillar crosssection, pillar aspect ratio, and gap distance. A hierarchical multi-pillar array design is proposed based on analysis of these variables. Figure 4.1 shows a schematic of the fabricated pressure sensor and Table 4.1 shows the test matrix. 
Table 4.1 Geometric specifications for all devices fabricated and tested.

\begin{tabular}{|c|c|c|c|c|c|c|}
\hline \multirow{2}{*}{ Sample \# } & \multirow{2}{*}{$\begin{array}{l}\text { Cross } \\
\text { section } \\
\text { shape }\end{array}$} & \multirow{2}{*}{$\begin{array}{c}\text { Aspect } \\
\text { ratio }\end{array}$} & \multicolumn{3}{|c|}{ Dimension $(\mu \mathrm{m})$} & \multirow{2}{*}{$\begin{array}{c}\text { Film } \\
\text { thickness } \\
(\mu \mathrm{m})\end{array}$} \\
\hline & & & gap & height & width & \\
\hline 1 & \multirow{9}{*}{ Square } & $1: 1$ & 100 & 100 & 100 & \multirow{14}{*}{200} \\
\hline 2 & & $1: 2$ & 100 & 200 & 100 & \\
\hline 3 & & $1: 3$ & 100 & 300 & 100 & \\
\hline 4 & & $1: 1$ & 200 & 100 & 200 & \\
\hline 5 & & $1: 3$ & 35 & 105 & 35 & \\
\hline 6 & & $1: 5$ & 21 & 105 & 21 & \\
\hline 7 & & $1: 7$ & 15 & 105 & 15 & \\
\hline 8 & & $1: 6$ & 17.5 & 105 & 17.5 & \\
\hline 9 & & $1: 9$ & 11.7 & 105 & 11.7 & \\
\hline \multirow{3}{*}{10} & \multirow{3}{*}{ Hybrid } & $1: 3$ & 145 & 100 & 33.3 & \\
\hline & & $1: 5$ & 65 & 80 & 16 & \\
\hline & & $1: 7$ & 14 & 60 & 8.6 & \\
\hline 11 & \begin{tabular}{|l|} 
Triangle \\
\end{tabular} & $1: 1$ & 48 & 100 & 152 & \\
\hline 12 & Circle & $1: 1$ & 87.2 & 100 & 112.8 & \\
\hline
\end{tabular}

\begin{tabular}{|c|c|c|c|c|c|c|}
\hline Sample \# & $\begin{array}{c}\text { Cross } \\
\text { section } \\
\text { shape }\end{array}$ & OR:IR & IR & OR & gap & height \\
\hline 13 & \multirow{6}{*}{$\begin{array}{c}\text { Half } \\
\text { Circle } \\
\text { (Easy } \\
\text { to } \\
\text { buckle) }\end{array}$} & $5: 3$ & 42.3 & 70.5 & 100 & 100 \\
\hline 14 & & $5: 4$ & 75.2 & 94.0 & 100 & 100 \\
\hline 15 & & $5: 3$ & 42.3 & 70.5 & 200 & 100 \\
\hline 16 & & $5: 4$ & 75.2 & 94.0 & 200 & 100 \\
\hline 17 & & $5: 3$ & 42.3 & 70.5 & 300 & 100 \\
\hline 18 & & $5: 4$ & 75.2 & 94.0 & 300 & 100 \\
\hline
\end{tabular}


Figure 4.1 a) shows a schematic of the actual test setup. The sensor circuit (Figure 4.1 a)) is completed by clamping copper tape to the $\mathrm{Au}$ electrodes and connecting the tape to electrical leads of the capacitance measurement device. A Model 3000, GLK instruments, 7.4 Hz was used for the electrical measurements. Because the top sensor layer is made of PDMS, which is not rigid and may not be flat, a glass slide is used to improve contact, distribute the load and ensure uniform pressure application. The glass slide covered the same unit cell (area) during all tests regardless of the design (Figure $4.1 \mathrm{~b}$ )).
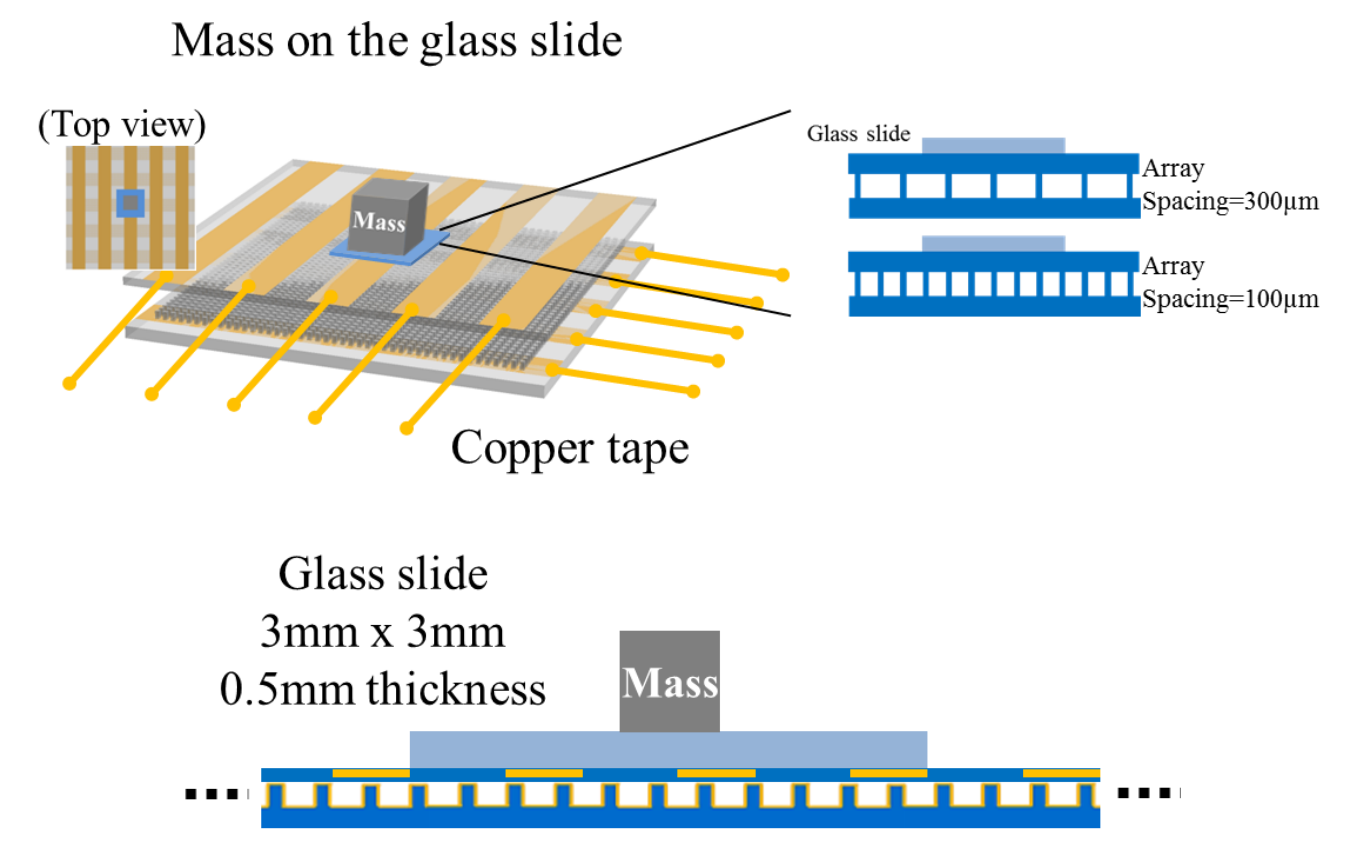

Figure 4.1 a) (Top) Test schematic and b) (bottom) Constant test area for the different tested sensor array designs.

\subsection{Micropillar array characteristics}

Each micropillar array was analyzed by SEM after fabrication to confirm structural features before the electroding process. Optical images of the different sensor designs are shown in Figures 4.2-4.4. Figure 4.2 shows the different cross-sections tested. The square, circle, and triangle cross-sections were fabricated whilst maintaining constant area. The pillars are regular and uniform as noted by the identical parallel rows in the SEM images. The two half-circle designs had varying inner and outer diameters as 
shown in Figure 4.2. Figure 4.3 shows rectangular cross-section arrays with two of the three different gap distances tested for an aspect ratio of 1:1.
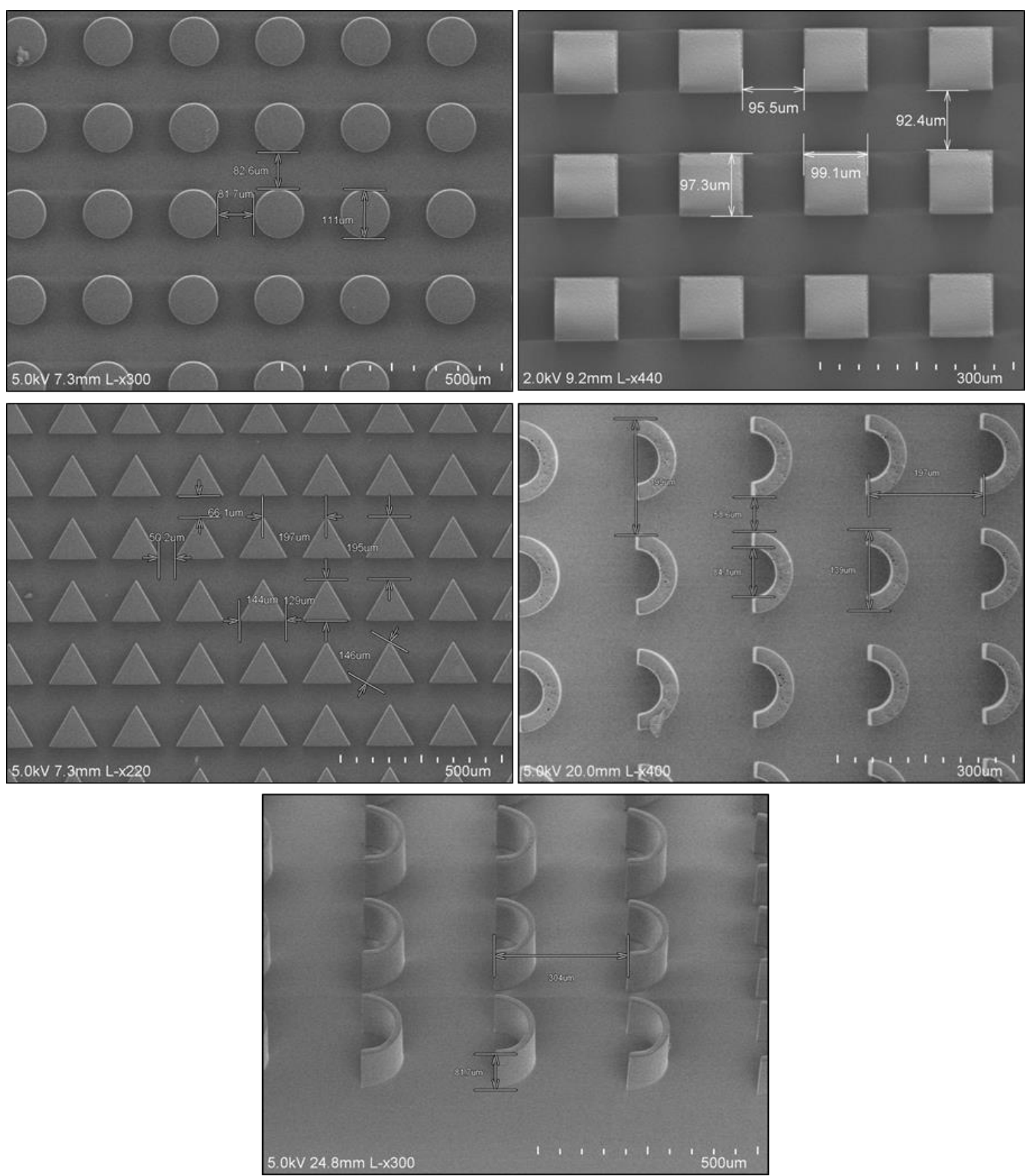

Figure 4.2 1:1 $100 \mu \mathrm{m}$ gap: square pillar, circle pillar, triangle pillar, half circle pillar. 

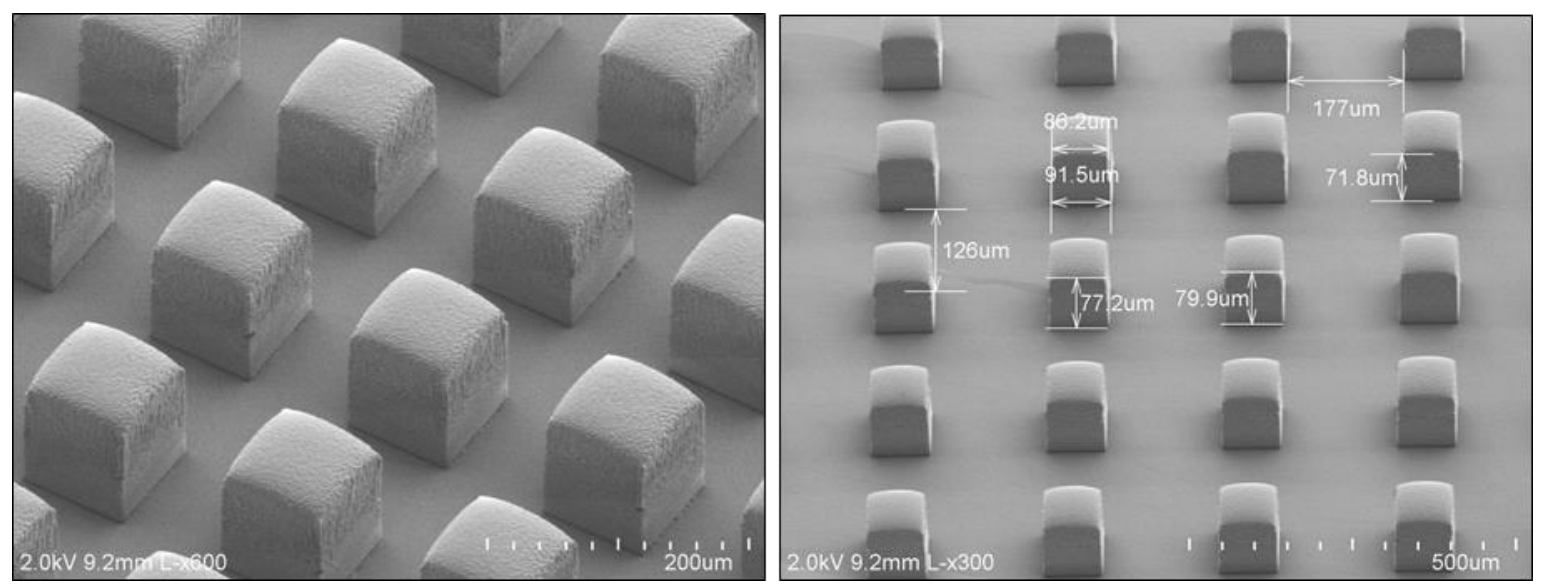

Figure 4.3 Gap distance, $1: 1$ with $100 \mu \mathrm{m}$ and $200 \mu \mathrm{m}$ gap.

Four different aspect ratios were tested and the SEM micrographs are shown in Figure 4.4. Theoretically, it can be shown that PDMS micropillars with a greater than 1:6 (Shu Yang 2006 Replica Molding of High-Aspect-Ratio Polymeric Nanopillar Arrays with High Fidelity) aspect ratio will collapse due to the mechanisms of self-loading (weight) and surface adhesion to the relief structure during lift off from the mold, the former being the main mechanism [81]. With increasing aspect ratio, some of the pillars start to tip over and stick to one another. At the maximum fabricated aspect ratio of 1:9, all the pillars are completely collapsed and have the appearance of cooked noodles. The pillars can be reconstituted to a standing position by flooding the pillars with silicone oil and treating the surface with an oxygen plasma treatment. 

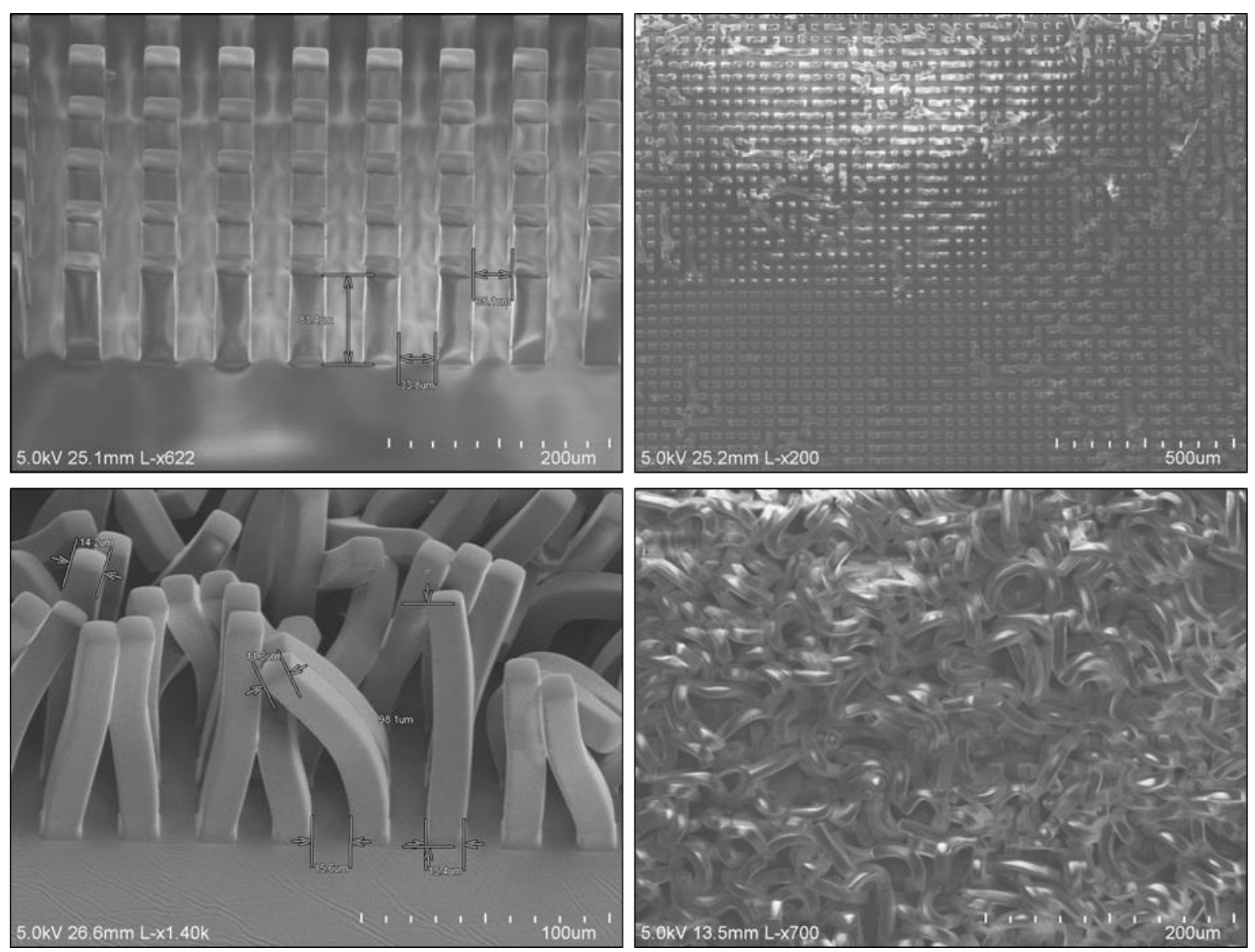

Figure 4.4. Aspect ratios from top to bottom - 1:3, 1:6, 1:7, and 1:9.

\subsection{Micropillar deformation}

\subsubsection{Influence of pillar imperfection on the deformation}

During the fabrication process, great care must be taken to minimize surface imperfections in order to produce a regular array that deforms uniformly and as expected. Closer examination of several initial batches revealed surface asperities and imperfections of 5\% over the range of pillar arrays fabricated. Typical surface asperities that occur on the tops of the pillars are shown in Figure 4.5. The surfaces are convex and have height differences of $1-3 \mu \mathrm{m}$ when compared to the edge height. Table 4.2 details the fabrication accuracy of the linear dimensions. The imperfections cause significant variations in the deformation response of the pillars as will be detailed below. This means that limits need to be set on the acceptable tolerances. 
Table 4.2 Nanofabrication accuracy before improving fabrication process.

\begin{tabular}{|c|c|c|c|c|}
\hline Design & Height & Width & OR & IR \\
\hline $\mathrm{Sq}$ & $100 \pm 4.2$ & $100 \pm 1.1$ & N/A & N/A \\
\hline $\mathrm{Cir}$ & $100 \pm 4.5$ & $112.84 \pm 1.3$ & N/A & N/A \\
\hline $\mathrm{Tri}$ & $100 \pm 3.8$ & $151.97 \pm 2.9$ & N/A & N/A \\
\hline $\mathrm{HCa}$ & $100 \pm 2.3$ & N/A & $42.31 \pm 1.7$ & $70.52 \pm 1.5$ \\
\hline $\mathrm{HCb}$ & $100 \pm 2.5$ & N/A & $75.23 \pm 1.9$ & $94.03 \pm 1.8$ \\
\hline
\end{tabular}

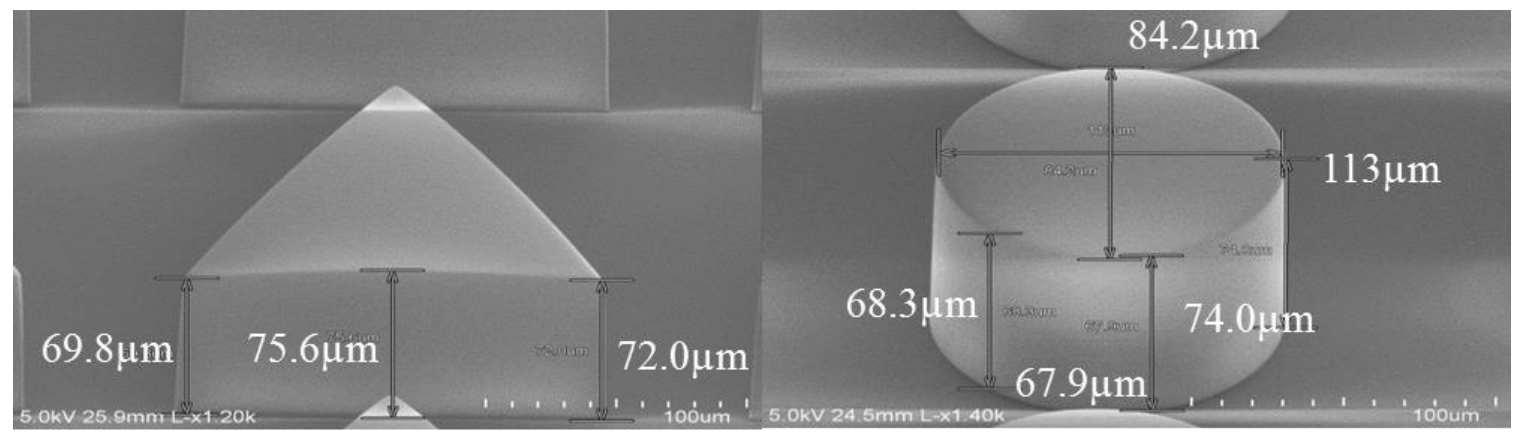

Figure 4.5 The pillar surface is not flat and planar.

The asperities and imperfections in pillar fabrication can lead to non-symmetric loading conditions, which affect the repeatability of array deformation response. The imperfections can be minimized by anisotropic etching. The variance in the pillar response was most severe for triangular shaped cross-sections. It is expected that at low to intermediate loads the pillars would deform by uniaxial compression. In the experimental test setup, a flat rigid plate is placed on top of the array to evenly distribute the load. Figure 4.6 shows the mixed mode deformation of asymmetric tilting in combination with compression and bending. (The image quality is not very good in the figure). It was difficult to deform the arrays in situ under the optical microscope. In addition to issues with positioning and lighting, the microscope camera was also poor.) The pillars tilt and bend because the non-planar top surface (imperfection caused by the fabrication) creates a load transfer point that is not through the centroid of the crosssection. Given the limitations of the test setup, the influence of the imperfections on pillar deformation was investigated using the finite element software ABAQUS. Single PDMS 
pillars with nonplanar surfaces (similar to a planar surface not loaded through the centroid) were simulated. Figure 4.7 shows the expected tilting and bending deformation of the pillar. The similarities between the experiment optical images and the simulation are clear and provided evidence for refining the fabrication technique. The improvements led to uniformity in the pillar deformation response, which is detailed in the next subsection.
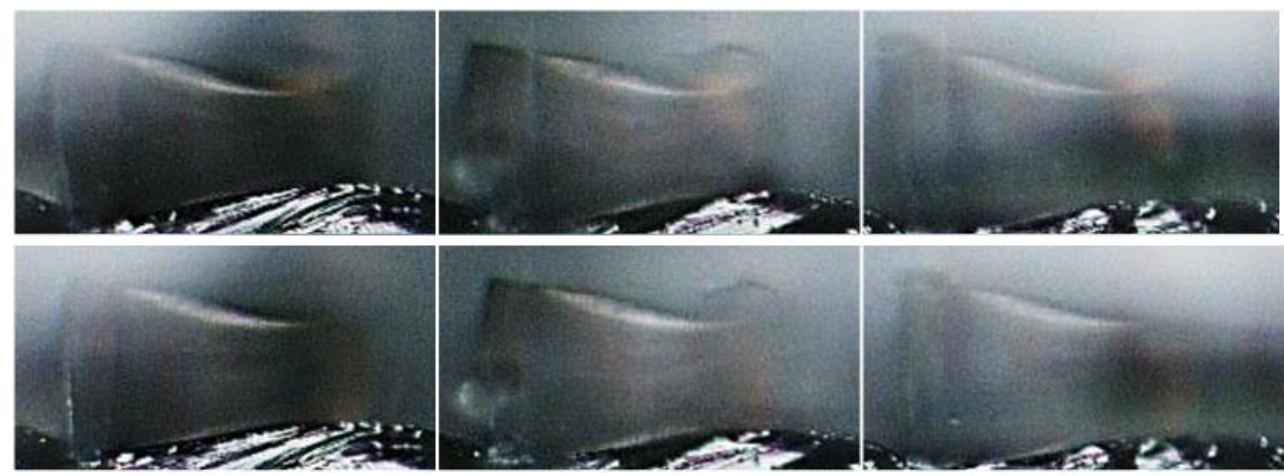

Figure 4.6 Tilting and bending of pillars - experimental results.
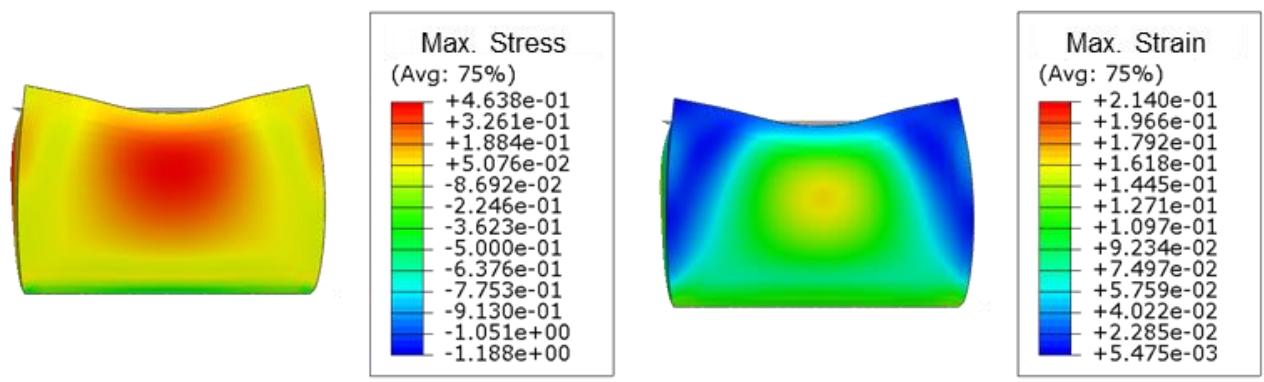

Figure 4.7 Tilting and bending of pillars - model simulation results showing stress (left) and strain (right) fields.

\subsubsection{Micropillar deformation of optimized pillar geometries}

In this section, experimental results are presented for the new sensor designs. With the updated fabrication technique, pillar geometries were fabricated within tolerances of $\pm 5 \%$. The surface of the mold is examined by a non-contact surface profiler to visualize the surface topology in 3D [82]. It is used to check the depth of the fabricated mold and dimensions of the pattern on it. There is a good match between the pattern image from 
the samples and the original design of the Cr mask. The pillar designs can be grouped into low aspect ratio pillars with symmetric cross-sections and high aspect ratio pillars with asymmetric cross-sections. Generally for low aspect ratio pillars, the top of the pillar makes increasing contact with the PDMS film and the pillar undergoes uniaxial compression (narrowing the gap between electrodes), as the load is increased further the pillar bulges and the compression is nonuniform. Optical micrographs of the progression of the deformation are shown in Figure 4.8. This behavior is typical of pillar samples 1-5 (Table 4.1). By tailoring the geometry of the pillars, the deformation can be amplified in a target pressure regime hence leading to larger capacitance changes and increased sensor sensitivity. Based on detailed analysis of these preliminary results, two new pillar geometries are proposed. To amplify the deformation, the pillars are designed to bend and buckle at low pressures, hence successfully leading to improved sensitivity, and extending the sensor performance to the ultra low pressure regime $\sim 1 \mathrm{~Pa}$. 

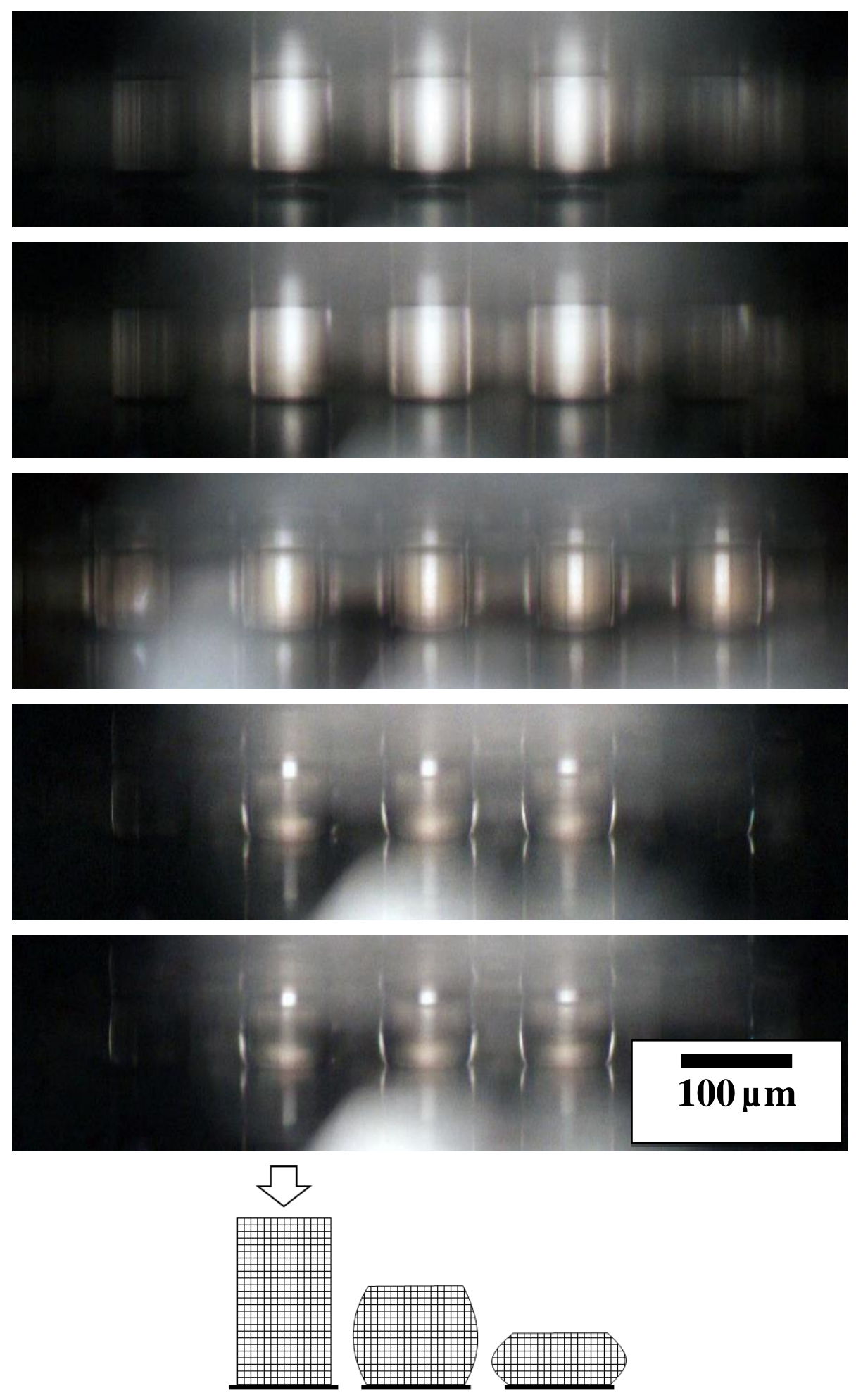

Figure 4.8 Progression of the deformation in low aspect ratio pillars and a schematic summary. 
Three pillar designs are introduced to increase sensor performance. High aspect ratio pillars are designed for bending, $\mathrm{C}$ shaped pillars are designed for elastic buckling at low pressures, and a hybrid pillar array composed of multi-level pillars was designed to maximize sensitivity using multiple contact levels through a combination of 3 high aspect ratio pillar designs. The hybrid arrays fabricated in this thesis all had square crosssections (Figure 4.9). It will be shown in the sensor results that the hybrid design had the highest sensitivity over all the pressure ranges tested followed by the high aspect ratio Cshaped pillar arrays. Figure 4.10 shows a series of experimental images illustrating the buckling response of the $\mathrm{C}$-shaped micropillars.
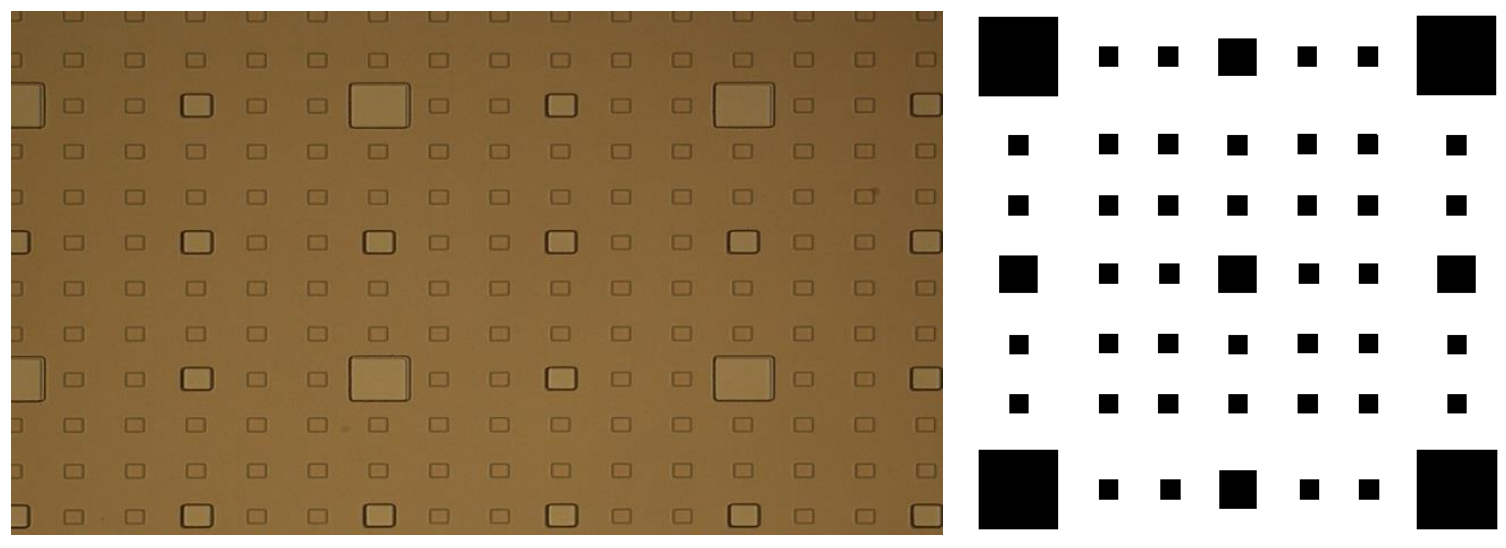

Figure 4.9 Top down picture (left) and schematic (right) of hybrid pillar array. 

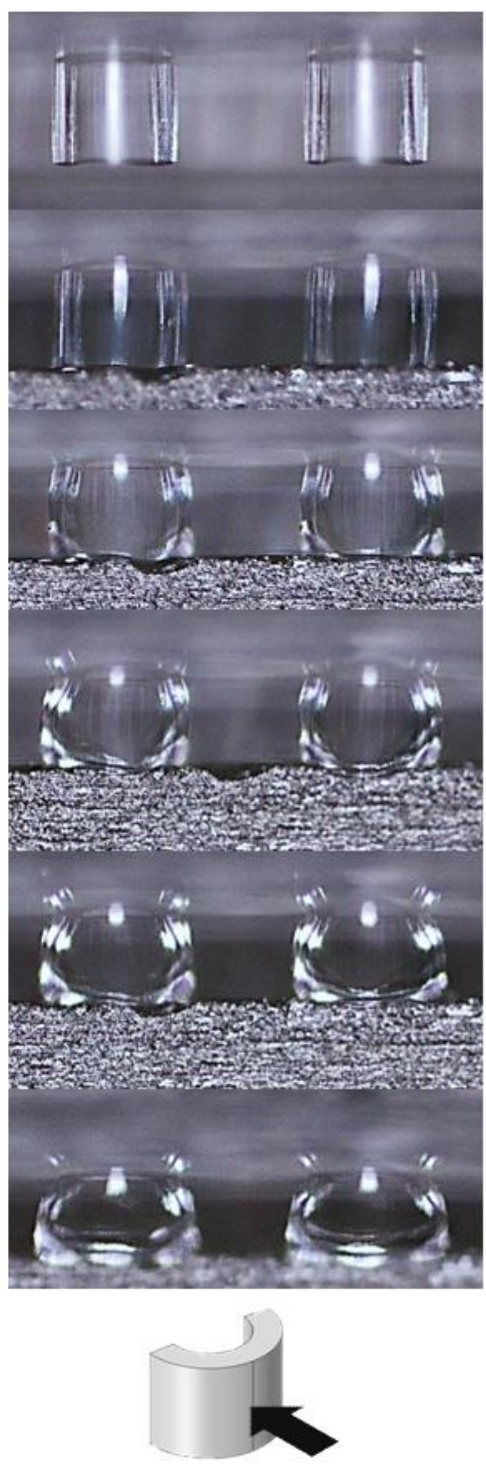
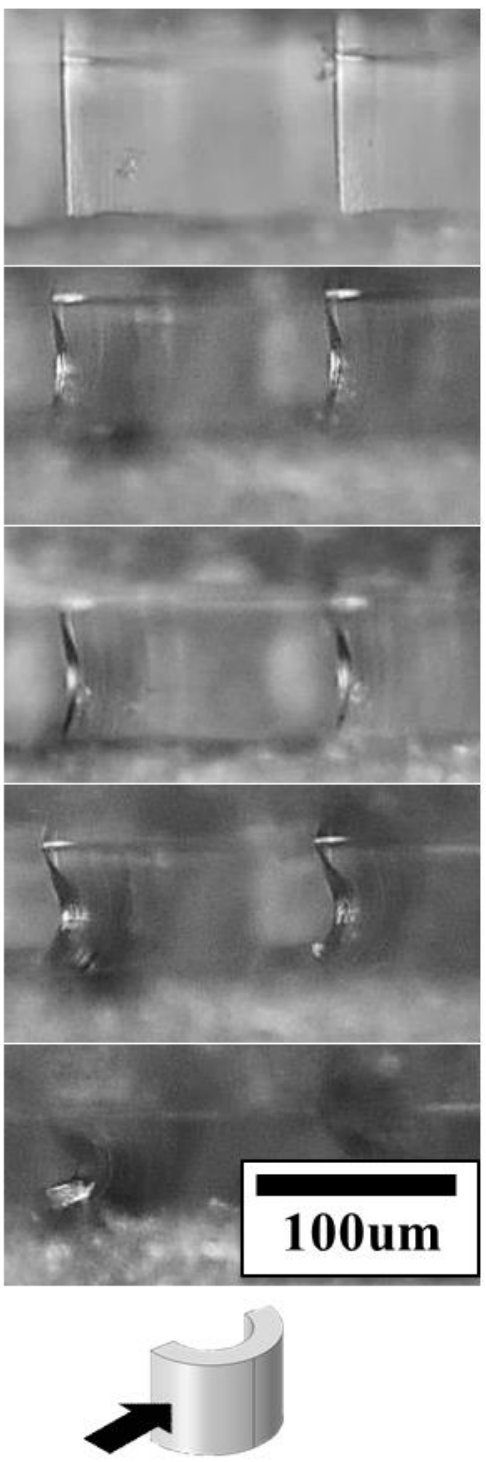

Figure 4.10 Optical images tracking the evolution of the deformation in C-shaped pillars under pressure loading. The arrows in the bottom schematic denote the viewpoint of the images above.

In summary, we designed micropillar arrays to tailor and optimize sensor functionality by taking of advantage of different deformation modes. A summary of the modes and corresponding pillar geometry is provided in Table 4.3. The hybrid design is a multiplatform array containing different pillar aspect ratios and gap distances as shown in the schematic of Figure 4.9. 
Table 4.3 Mechanism and Functionality of Micropillar Sensor Arrays.

\begin{tabular}{|c|c|c|c|}
\hline $\begin{array}{l}\text { Design } \\
\text { Deformation } \\
\text { mechanism }\end{array}$ & $\begin{array}{l}\text { Gap distance } \\
\text { (GD) }\end{array}$ & $\begin{array}{l}\text { Aspect ratio } \\
\text { (AR) }\end{array}$ & Cross section \\
\hline Pure compression & $100 \sim 300 \mu \mathrm{m}$ & Normally $1: 1$ & Square \\
\hline Barreling & $100 \sim 300 \mu \mathrm{m}$ & $1: 1 \sim 1: 3$ & Square \\
\hline Buckling & $100 \sim 300 \mu \mathrm{m}$ & $\begin{array}{c}\text { Above } 1: 3 \text { or easy to } \\
\text { buckle design }\end{array}$ & Square, Half circle \\
\hline Hybrid & $\begin{array}{l}\text { Combined GDs } \\
(100 \sim 300 \mu \mathrm{m})\end{array}$ & $\begin{array}{c}\text { Combined } \\
\text { ARs }(1: 1 \sim 1: 9)\end{array}$ & Square \\
\hline
\end{tabular}

\subsection{Sensor response}

The sensor response was measured experimentally and also modeled in COMSOL for comparison purposes. The experimental results are presented in this chapter and the computational analysis in the following chapter (Chapter 5). For the sensor experiments, we focus on two basic cross-sections and vary the aspect ratio and gap distance. The hybrid design is a hierarchical array and hence a special case. The sample specifications for all devices fabricated and tested are outlined in Table 4.1. For each case, three samples were tested three times and the average reported. Figure 4.11 illustrates the cyclic off and on response of the capacitive micropillar array. 


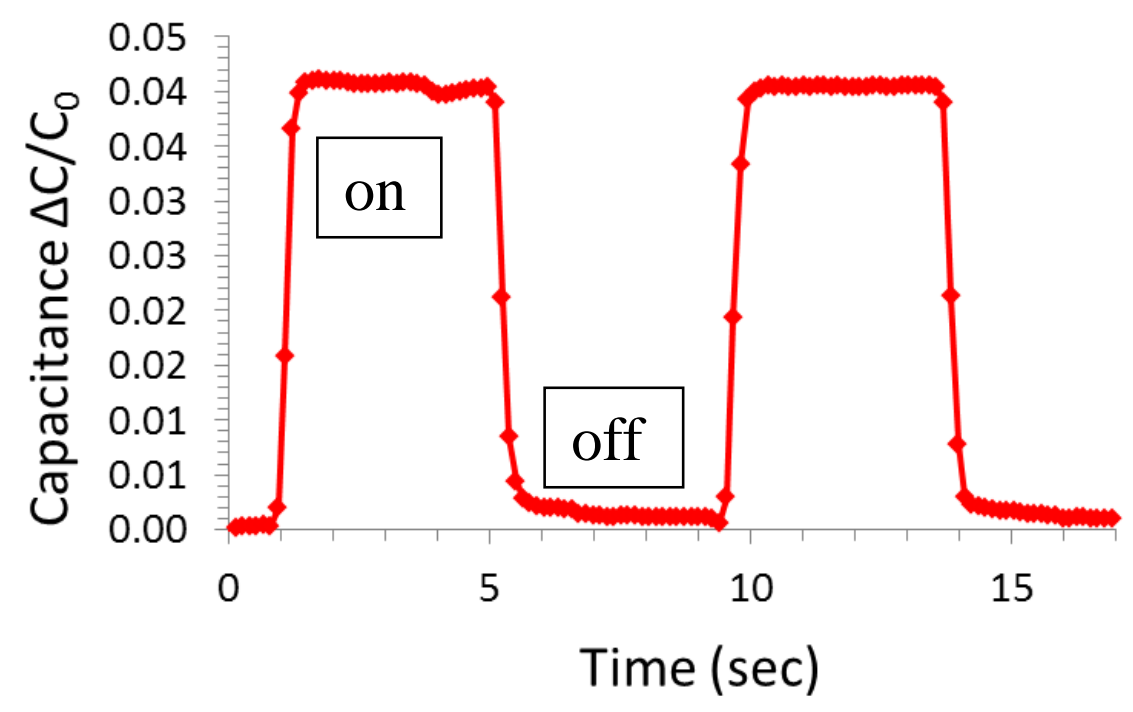

Figure 4.11 Cyclic response of sensor array when a $5 \mathrm{~g}$ weight is applied.

Precise capacitance measurements were made with a table-top capacitance meter (Model 3000, GLK instruments, $7.4 \mathrm{~Hz}$ ). The response time of the sensor was measured using a capacitance to digital converter (EVAL-AD7746EBZ, Analog Devices) with a $90 \mathrm{~Hz}$ sampling rate. The sensor response for 3 different cross-sections of identical area is shown in Figure 4.12 (cir - circle, sq - square, and tri-triangle). The results are plotted as the relative capacitance versus applied pressure. The behavior is quite similar over the range of pressures tested and so in the following, we focus on a single cross-section design. 
(a)
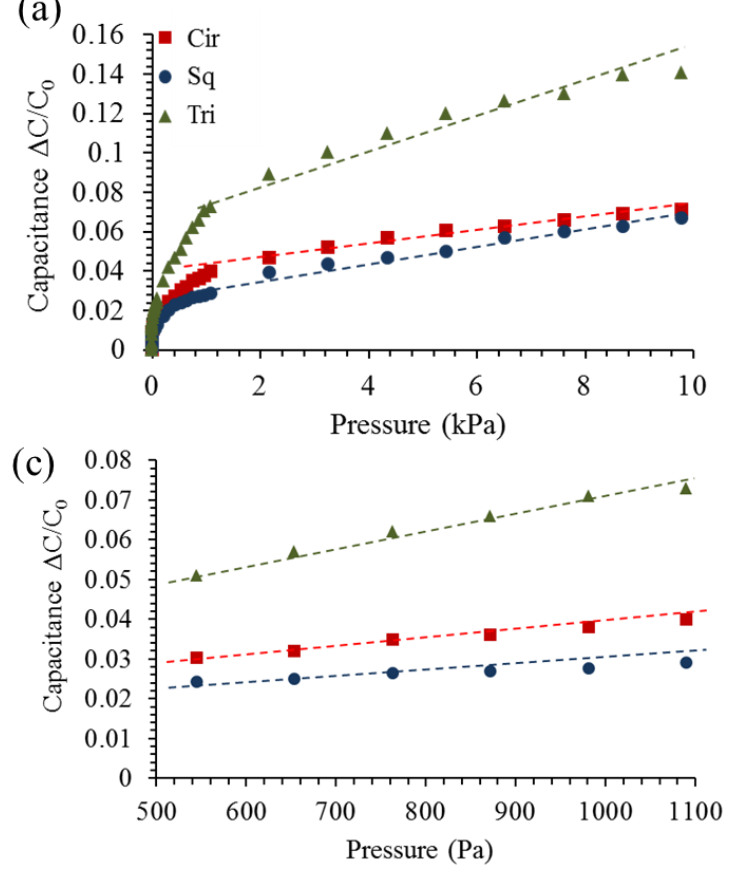

(b)

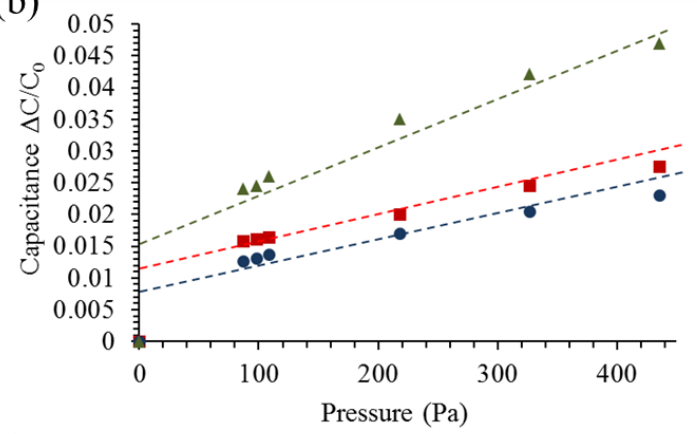

(d)

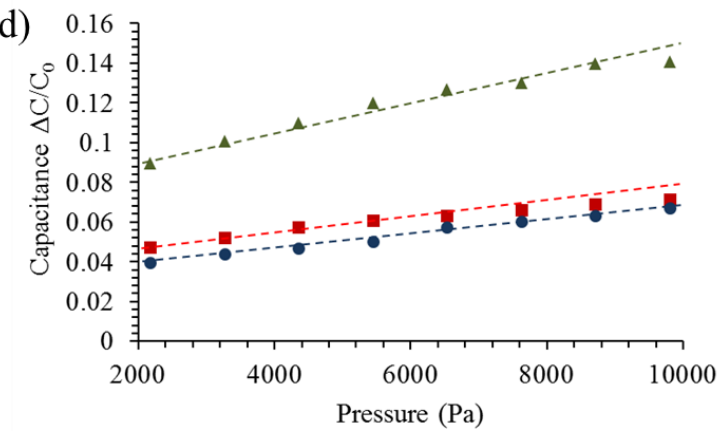

Figure 4.12 Sensor response for different cross-sections (constant area).

Figure 4.13 shows the measured static capacitance response for three different pillar aspect ratios having a square cross-section. We can identify three linear sensing regimes classified by the pressure range and corresponding sensitivity: (b) low pressure (80 to 500 $\mathrm{Pa}$ ), (c) medium-low pressure (500 Pa to $2 \mathrm{kPa}$ ), and touch (over $2 \mathrm{kPa}$ and <10 $\mathrm{kPa}$ ). The ultra low sensing range $<80 \mathrm{~Pa}$ was actually ruled out as due to proximity sensing detecting object placement versus actual pressure sensing. This range is investigated further using computational simulations detailed in Chapter 5. The lowest detectable pressure will depend on sensor design. Generally, for the sensors fabricated in this study a low limit of $80 \mathrm{~Pa}$ was determined. Two major mechanisms are responsible for the various pressure sensing regimes: fringe field effect and pillar deformability. Upon initial loading $(10 \mathrm{~Pa})$, the tops of the pillars are increasingly in contact with the soft upper film. Upon further loading, the pillars axially compress and the distance between the pillar and film decreases (electrode surfaces are brought into closer proximity) and the pillar further deforms by bulging. The elastic deformation continues for pressures up to approximately $800 \mathrm{~Pa}$, the pillar has a stiffening response in the limit of its bulk resistivity leading to the low sensitivity regime. For this range of aspect ratios (1:1 to $1: 3)$, we see that both the 
sensitivity and capacitance change decreases with increasing aspect ratio. This occurs because the micropillar structure is stiffer with increased aspect ratio for the range and architecture considered in his work (sample 1-3 in Table 4.1). Increased stiffness means less deformation (gap change) and hence smaller changes in capacitance.

Of particular interest is the low pressure regime, which shows the highest sensitivity reported to date for a passive capacitive sensor. The lowest detectable pressure using the current architectures is $80 \mathrm{~Pa}$. This means the sensing range (good repeatability) is $80 \mathrm{~Pa}$ to over $10 \mathrm{kPa}$. This is considered a wide sensing regime with low pressure detectability. The idea that the ultra-low pressure is in part due to a disturbance in the fringe field means a reclassification of the pressure response as a proximity sensor (object presence detection versus object detection through contact, which would be a true pressure reading). It should be noted that detecting pressures down to $0.9 \mathrm{~Pa}$ and $3 \mathrm{~Pa}$ have been reported by [60] and [51].
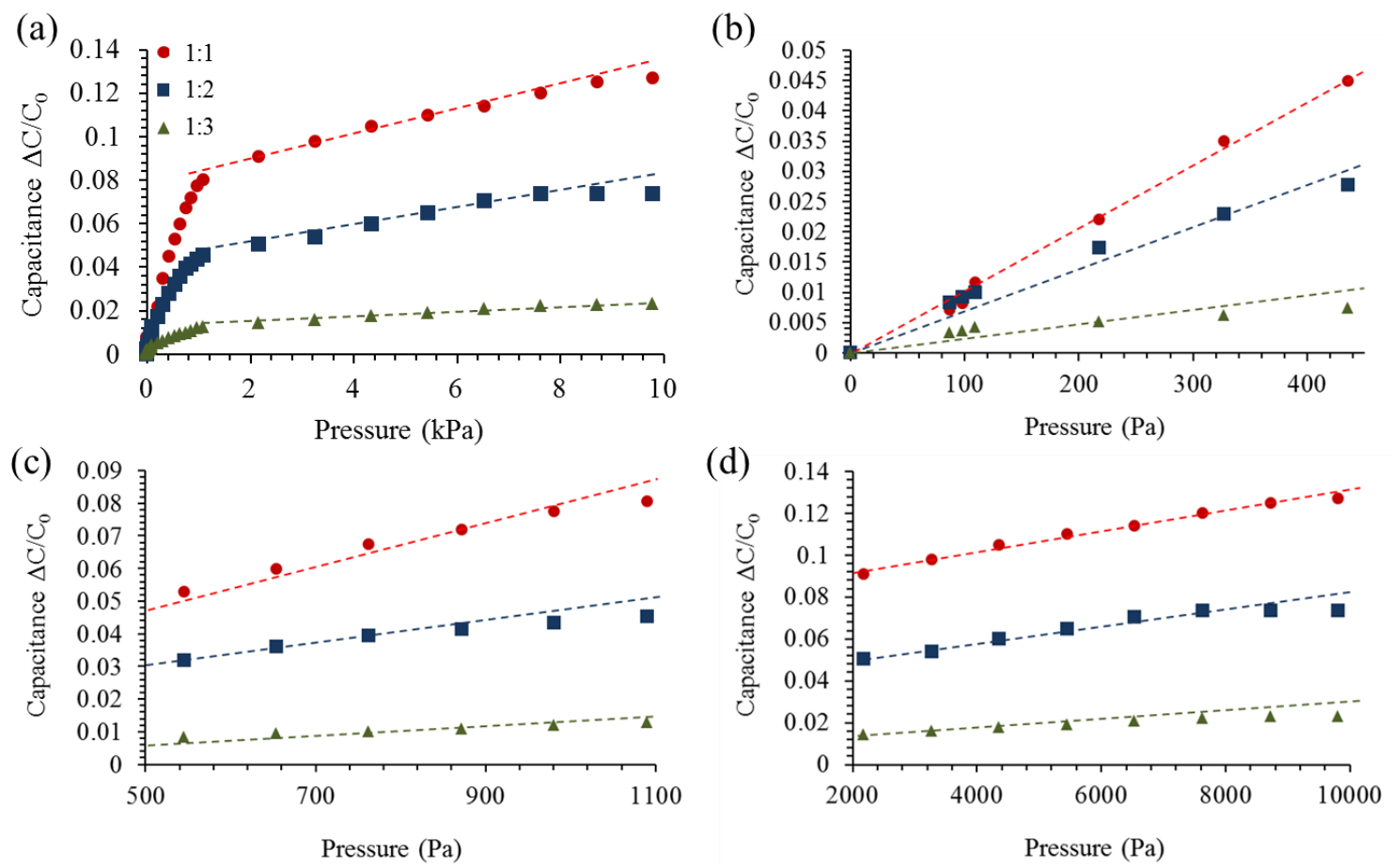

Figure 4.13 Sensor capacitance response for three different pillar aspect ratios. 
Experimental results for the half circle design are reported in Figure 4.14 and compared with the square cross section pillars with a 1:1 aspect ratio. The results clearly show improved sensitivity over the entire pressure range for the easy to buckle half circle designs.

(a)
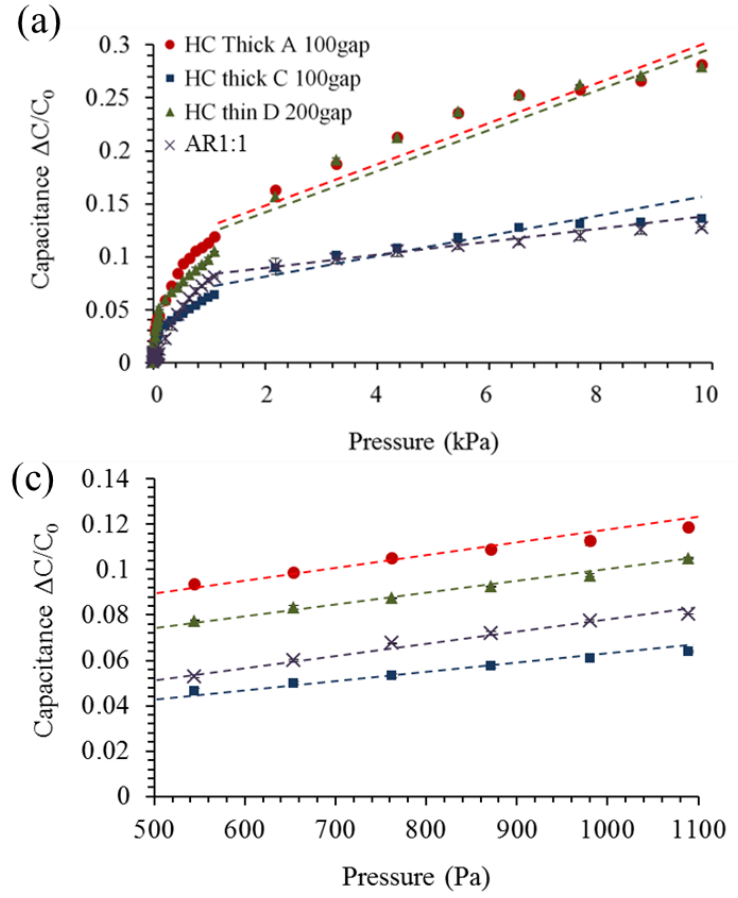

(b)

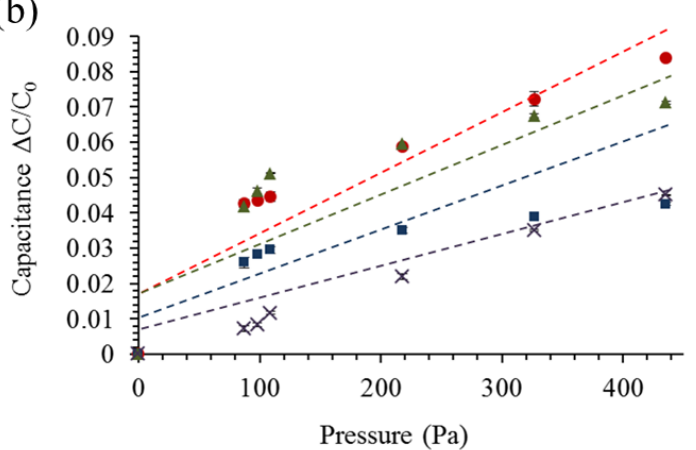

(d)

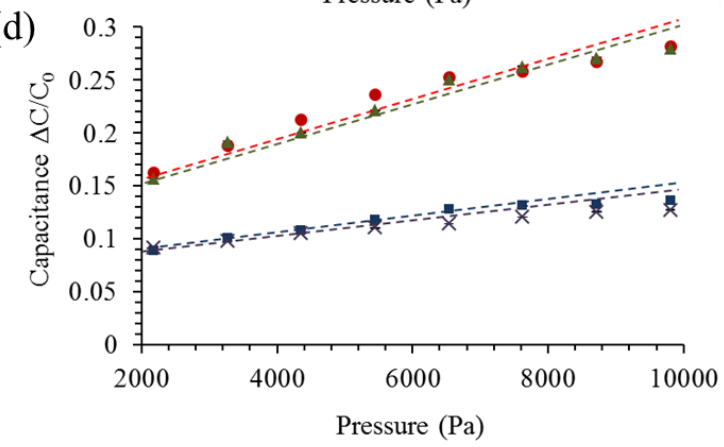

Figure 4.14 Sensor capacitance response for three different Half circle designs.

Figure 4.15 shows a comparison between the best buckling designs (from Figure 4.14) and the proposed hybrid sensor design. The results clearly show that sensor sensitivity is improved over the entire range for the hierarchical design. Sensitivity is increased because of the lowered effective stiffness of the array and local changes in the dielectric permittivity brought about by the varying gap between a subset of pillars and the top monolithic PDMS film. 
(a)
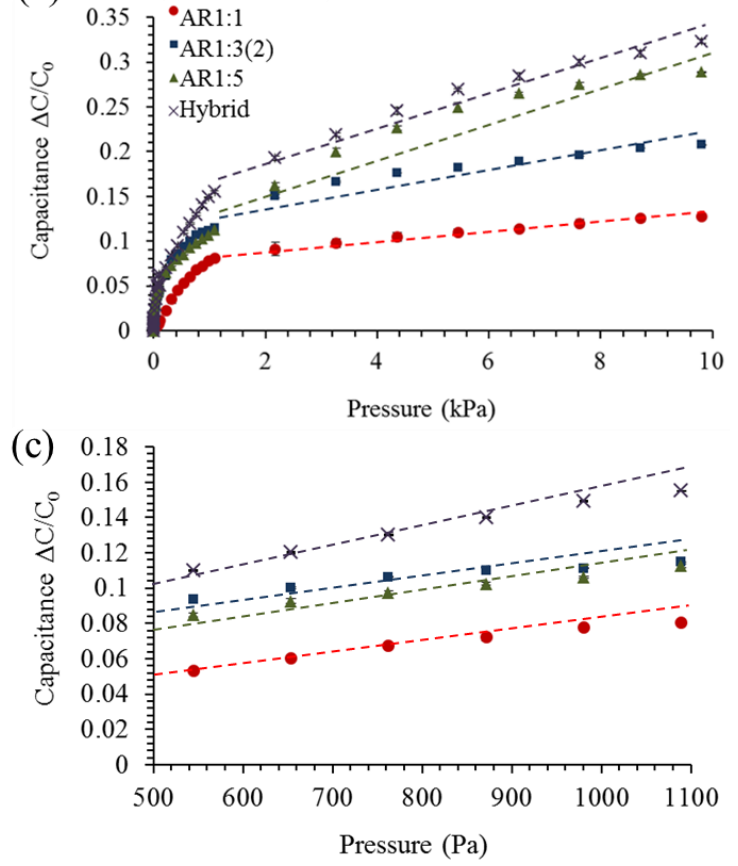

(b)

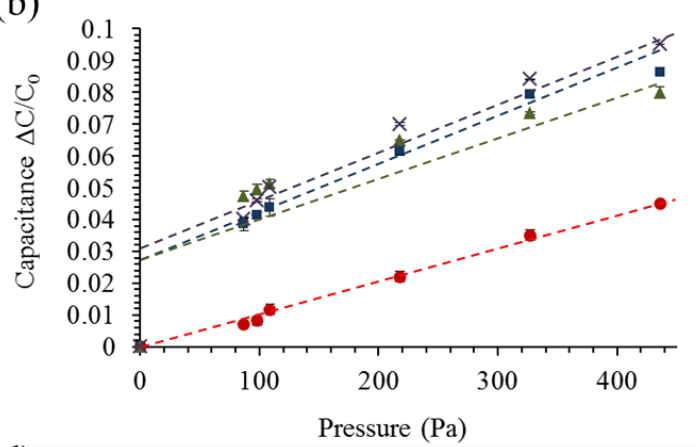

(d)

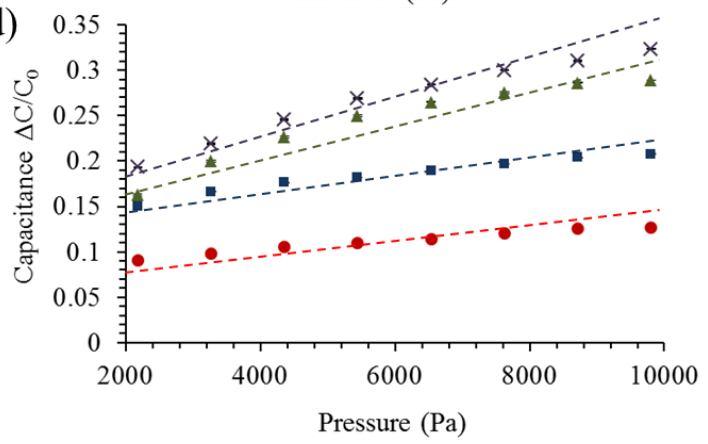

Figure 4.15 Sensor capacitance response for Hybrid with three different pillar aspect ratios.

Sensor sensitivity is calculated to assess overall performance. This is evaluated using the expression in (4.1), where $\Delta \mathrm{C}$ is the capacitance change, $\mathrm{C}_{0}$ is the initial capacitance, and $\mathrm{p}$ is prescribed pressure

$$
S=\frac{\delta\left(\Delta \mathrm{C}_{/} / \mathrm{C}_{0}\right)}{\delta p}=\frac{1}{\mathrm{C}_{0}} * \frac{\delta \mathrm{C}}{\delta p}
$$

The sensitivity is tabulated in the different pressure regimes for each of the designs (Table 4.4). 
Table 4.4 Sensitivity $\left(\mathrm{kPa}^{-1}\right)$ in the different pressure regimes for each of the designs.

\begin{tabular}{|l|c|c|c|}
\hline Micropillar design & $\mathbf{8 0 - 5 0 0 ~ P a}$ & $\mathbf{5 0 0}$ Pa-2 kPa & $\mathbf{2 - 1 0 ~ k P a}$ \\
\hline $1: 1$ & 0.10 & 0.08 & 0.010 \\
\hline $1: 2$ & 0.06 & 0.04 & 0.006 \\
\hline $1: 3$ & 0.01 & 0.02 & 0.003 \\
\hline 1:3(2) & 0.13 & 0.11 & 0.020 \\
\hline 1:5 & 0.24 & 0.10 & 0.030 \\
\hline HC A 100 gap & 0.21 & 0.12 & 0.020 \\
\hline HC C 100 gap & 0.11 & 0.08 & 0.010 \\
\hline HC D 200 gap & 0.10 & 0.09 & 0.020 \\
\hline Hybrid & 0.34 & 0.14 & 0.030 \\
\hline
\end{tabular}

\subsection{Time scale characteristics of sensor response}

The time scale characteristics of the micropillar array was measured using a digital converter (EVAL-AD7746EBZ, Analog Devices) circuit. Figure 4.16 shows a schematic of the circuit [83]. For a typical sensor of square geometry and 1:1 aspect ratio, the sensor response time was $60.8 \mathrm{~ms}$. The response time is defined as the time interval between $10 \%$ and $90 \%$ of the steady state values.[84]. Figure 4.17 a) shows the typical input/output traces between the applied force and the measured capacitance change. In Table 4.5, the response time is compared with other flexible sensor systems. And Table 4.5 shows a comparison of the sensor developed here with other microstructured sensor platforms. 

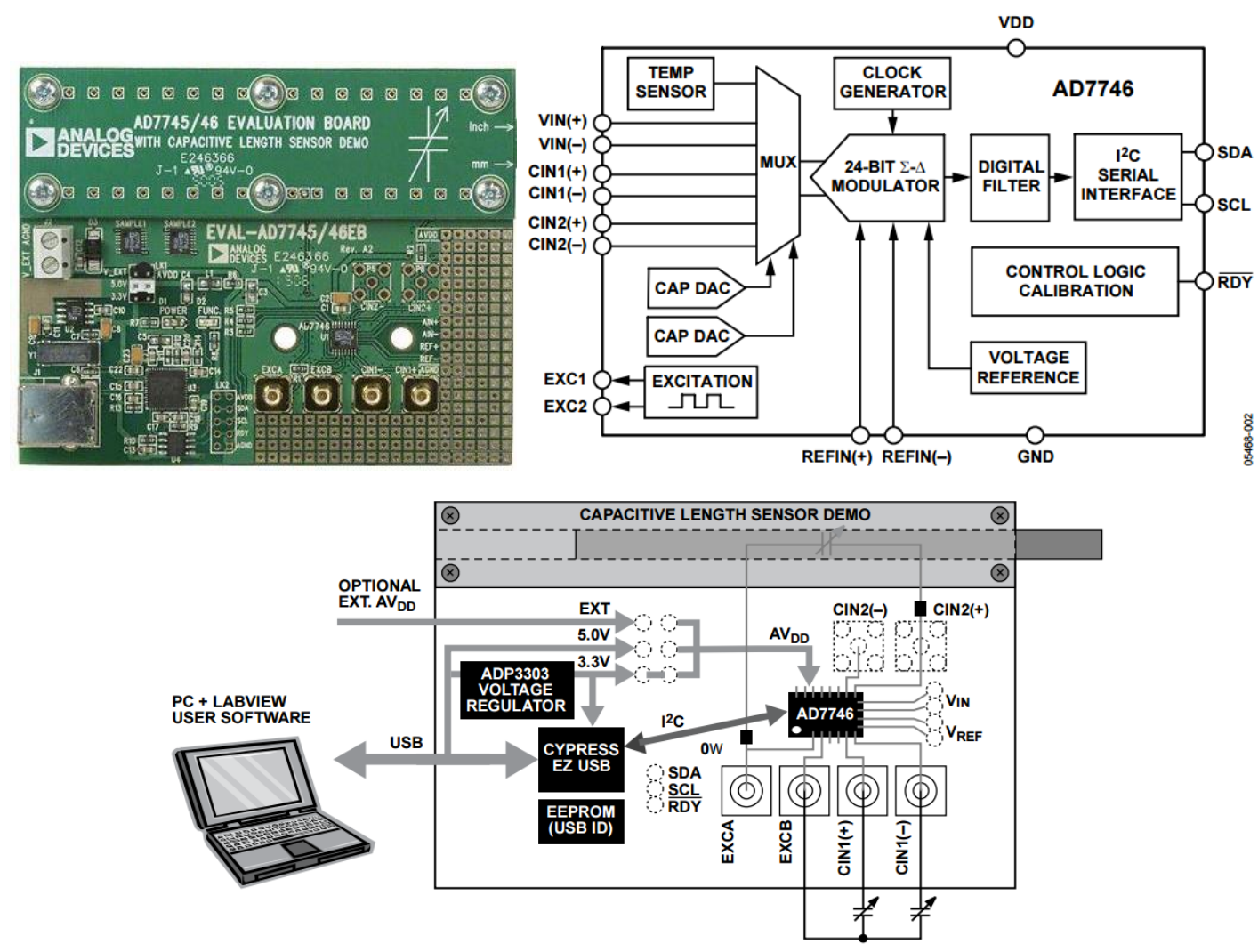

Figure 4.16 Circuit used to measure sensor response time taken from Ref [83].

Time vs. Capacitance and Force

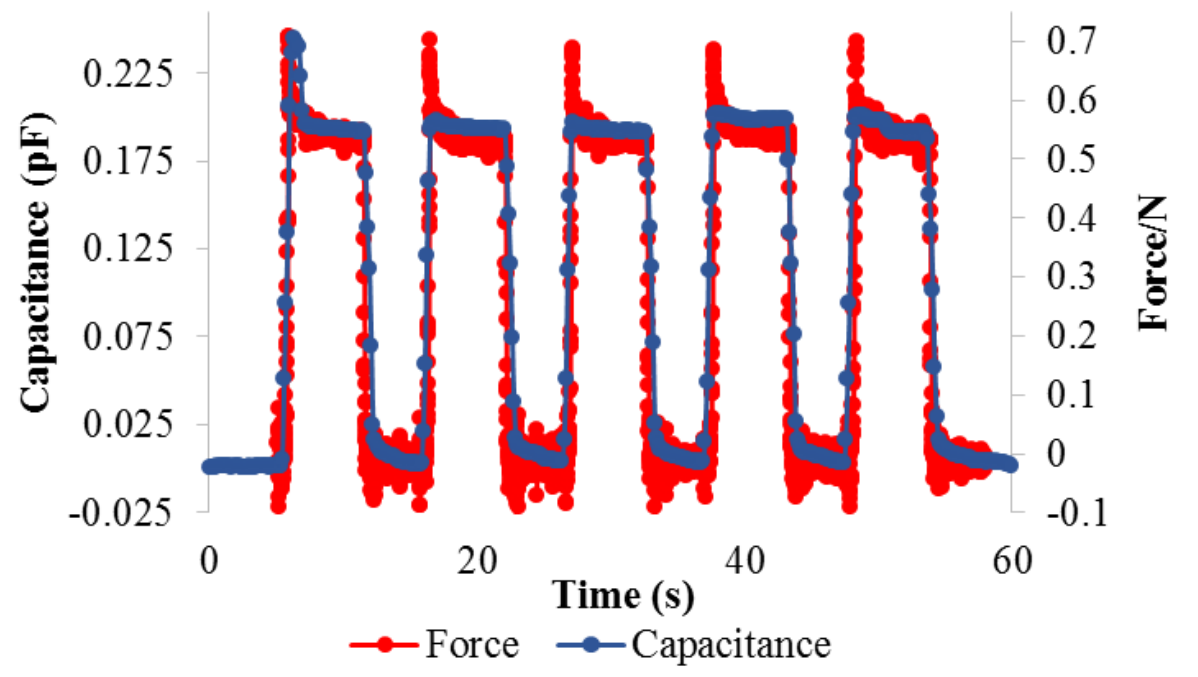




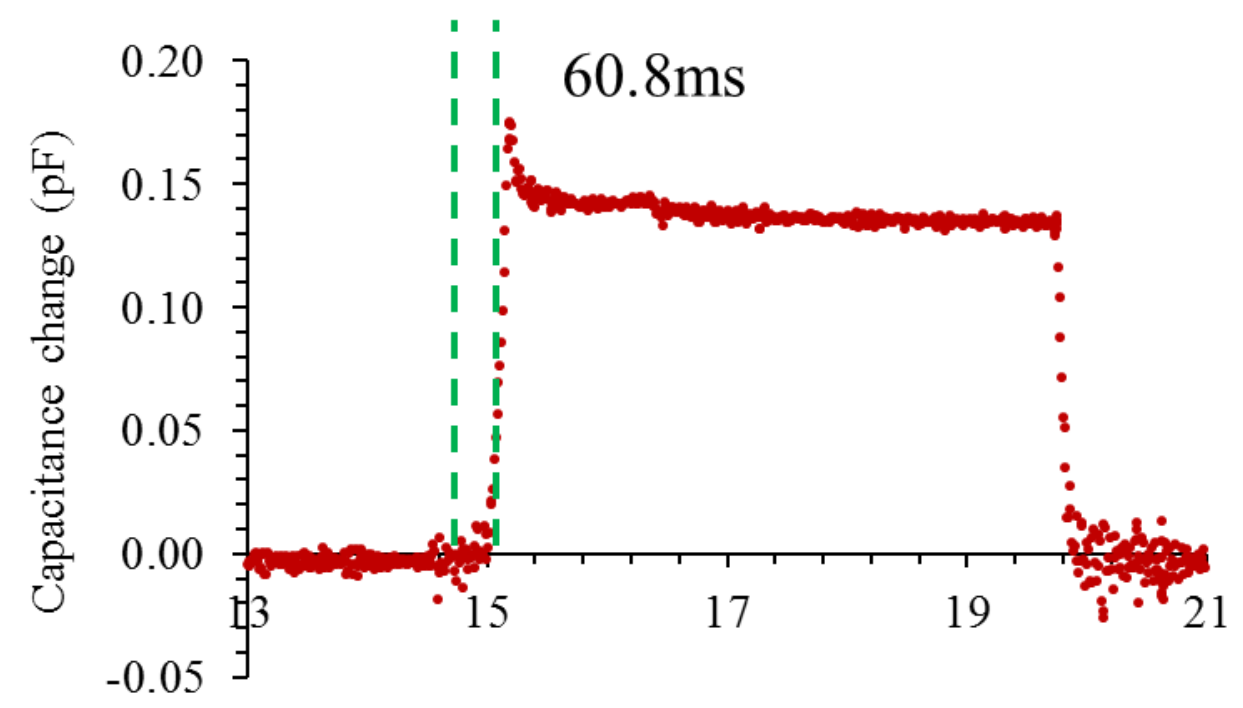

Time (s)

Figure 4.17 1:1 AR Sq. 200 $\mu$ m gap micropilar a) Capacitance and force versus time graph. B)

Capacitance versus time graph. The response time is $60.8 \mathrm{~ms}$.

Table 4.5 Comparison of the proposed sensor and other flexible sensor systems.

\begin{tabular}{|c|c|c|c|c|}
\hline Reference & $\begin{array}{c}\text { Sensing } \\
\text { mechanism }\end{array}$ & Fabrication technique & $\begin{array}{c}\text { Sensitivity } \\
\left(\mathbf{k P a}^{-1}\right)\end{array}$ & $\begin{array}{c}\text { Response time } \\
(\mathbf{m s})\end{array}$ \\
\hline $\begin{array}{c}\text { Liu et al } \\
(2013)\end{array}$ & Resistive & $\begin{array}{c}\text { PDMS molding with Al } \\
\text { mold }\end{array}$ & 0.23 & $\begin{array}{c}175(\text { load }) \\
205(\text { unload) }\end{array}$ \\
\hline $\begin{array}{c}\text { Yao et al } \\
(2014)\end{array}$ & Capacitive & $\begin{array}{c}\text { Silicon based } \\
\text { micromachined (DRIE) }\end{array}$ & 0.0016 & 45 \\
\hline $\begin{array}{c}\text { Mannsfeld } \\
\text { et al (2010) }\end{array}$ & Capacitive & $\begin{array}{c}\text { Photolith. for SI mold, } \\
\text { PVD of Rubrene }\end{array}$ & 0.55 & $<200$ \\
\hline This work & Capacitive & $\begin{array}{c}\text { Soft lithography and } \\
\text { DRIE }\end{array}$ & 0.34 & 60 \\
\hline
\end{tabular}

\subsection{Sensor applications}

The micropillar array can be configured for different types of sensor applications. Here, we focus on two potential applications. The first is a sensor array for spatial or discrete sensing and secondly, an arterial blood pressure sensor. The preliminary results show that the sensor has good potential for both these applications. 


\subsubsection{Pixel-type sensors}

The micropillar sensor is configured for a pixel-type response by applying discrete electrodes. The overall dimension of the array is $20 \mathrm{~mm}$ by $20 \mathrm{~mm}$ and each pixel is 2 $\mathrm{mm}$ by $2 \mathrm{~mm}$. The limiting spatial resolution depends on the ability to discretize the electrodes. Pixel type sensors are fabricated using the method described in Chapter 3. Gold sputtered electrodes are applied in a grid pattern to create 25 capacitive cells or pixels. Copper tape connects to the Au electrodes and lead to the capacitance meter. Figure 4.19 shows the test setup with two rubber block letters ' $U$ ' and ' $M$ ' placed on the sensor device. The capacitance read out is shown below in the same figure. The highest capacitance cells (red and orange) give the pixelated letter response. In this test, the maximum capacitance change was $0.14 \mathrm{pF}$.

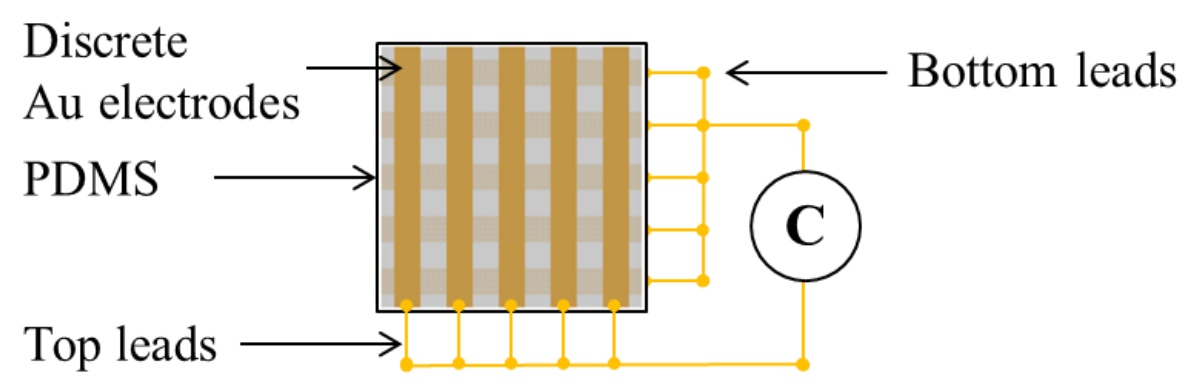

Figure 4.18 Multiplex processing circuit for multi-capacitor array readout. 

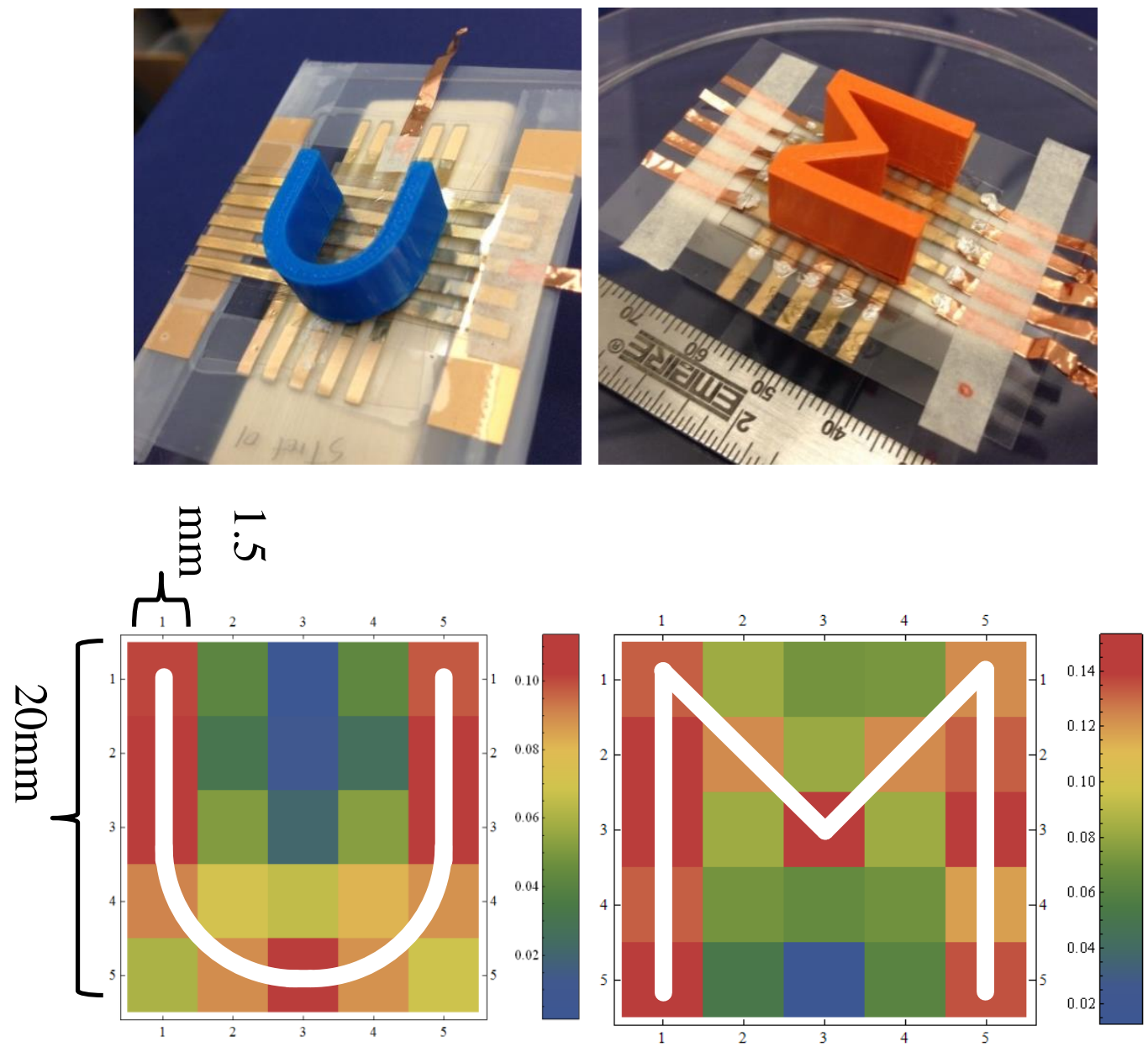

Figure 4.19 Spatial response of micropillar sensor array.

\subsubsection{Arterial blood pressure sensors}

Monitoring vascular tone continuously and noninvasively in a medical setting is a key area of interest in emergency medicine. The main requirements for the sensor are that it be lightweight, wearable (flexible), portable, sensitive, and have sufficient accuracy to detect modulations in blood pressure signals to various stages of unhealth. Medical conditions such as syncopy, sepsis, hypertension and intradialytic hypotension can be detected and predicted before they occur with such a monitoring system [85]. As current systems are bulky, stiff, and based on technologies that require large processing units, advancements in this area are critically needed. Here, we illustrate the use of the micropillar array for detecting pulse pressure by wrist measurement. (Micropillar array specifications: AR 1:2,100 $\mu \mathrm{m}$ square geometry, $100 \mu \mathrm{m}$ gap). Figure 4.20 shows the 
experimental test setup and output for arterial measurement on the wrist of a subject at rest. The trace shows the typical systolic and diastolic peak pattern.

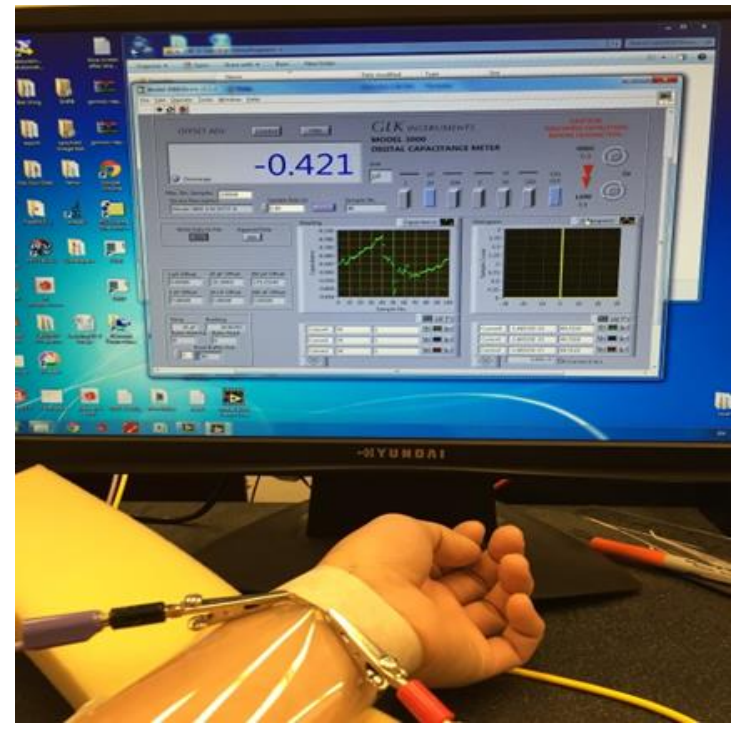

Time vs Capacitance

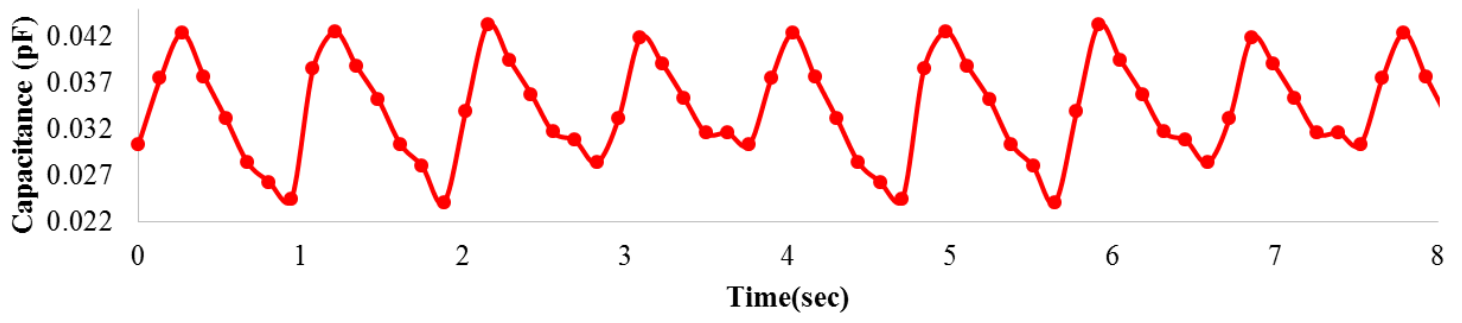

Time vs Pressure

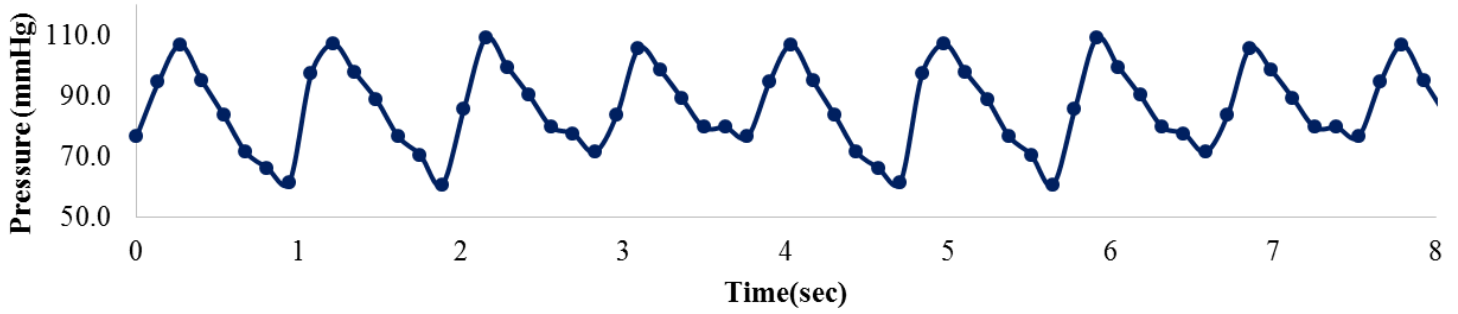

Figure 4.20 Arterial blood pressure by wrist measurement for subject at rest.

\subsection{Summary}

In this chapter, design characteristics of micropillar arrays and their sensing performance are presented and analyzed. Based off of preliminary designs, new high aspect ratio and 
easy to buckle designs are proposed and showed higher performance. Further insight to correlate pillar design with improved sensor sensitivity and increased sensor detection range is found using a multiphysics computational approach detailed in Chapter 5. 
Table 4.6 Comparison of microstructured sensors.

\begin{tabular}{|c|c|c|c|c|c|c|}
\hline Type & Structure & $\begin{array}{c}\text { Sensing } \\
\text { mechanism }\end{array}$ & $\begin{array}{c}\text { Fabrication } \\
\text { technique }\end{array}$ & $\begin{array}{c}\text { Sensitivity } \\
\left(\mathrm{kPa}^{-1}\right)\end{array}$ & Range & $\begin{array}{c}\text { Response } \\
\text { time }\end{array}$ \\
\hline Flexible & $\begin{array}{c}\text { Gold serpentine } \\
\text { pattern on PDMS }\end{array}$ & Resistive & $\begin{array}{l}\text { PDMS molding } \\
\text { with Al mold }\end{array}$ & 0.23 & $\begin{array}{c}1 \mathrm{~Pa} \sim \\
6.7 \mathrm{kPa}\end{array}$ & $60 \mathrm{~ms}$ \\
\hline Flexible & $\begin{array}{c}\text { OFETs - indium tin } \\
\text { oxide (ITO)-coated } \\
\text { flexible PET sheet } \\
\text { on top of } \\
\text { microstructured } \\
\text { PDMS }\end{array}$ & Capacitive & $\begin{array}{c}\text { Photolith. for SI } \\
\text { mold, PVD of } \\
\text { Rubrene }\end{array}$ & $\begin{array}{c}0.55 \\
(<2 \mathrm{kPa} \\
\text { regime })\end{array}$ & $\begin{array}{l}3 \mathrm{~Pa} \sim \\
7 \mathrm{kPa}\end{array}$ & $\sim 200 \mathrm{~ms}$ \\
\hline Flexible & $\begin{array}{l}\text { Ppy gel (conducting } \\
\text { polymer) }\end{array}$ & Piezo-Resistive & $\begin{array}{c}\text { Micropatterned } \\
\text { inverse mold }\end{array}$ & $\begin{array}{l}56-133 \\
(<30 \mathrm{~Pa} \\
\text { regime })\end{array}$ & $\begin{array}{c}0.8 \mathrm{~Pa} \sim \\
10 \mathrm{kPa}\end{array}$ & $50 \mathrm{~ms}$ \\
\hline Flexible & $\begin{array}{l}\text { Reversible } \\
\text { interlocking of } \\
\text { nanoofibres }\end{array}$ & Piezo-Resistive & $\begin{array}{c}\text { SI Master, PUA } \\
\text { mold }\end{array}$ & $\begin{array}{l}11.45 \\
\text { (Gauge } \\
\text { factor) }\end{array}$ & $\begin{array}{l}5 \mathrm{~Pa} \sim \\
1.5 \mathrm{kPa}\end{array}$ & N/A \\
\hline Rigid & $\begin{array}{l}\text { parylene-based } \\
\text { wireless pressure } \\
\text { sensor }\end{array}$ & $\begin{array}{c}\text { Capacitor/indu } \\
\text { ctor resonant } \\
\text { circuits }\end{array}$ & $\begin{array}{c}\text { Silicon based } \\
\text { micromachined } \\
\text { (DRIE) }\end{array}$ & 0.058 & $\begin{array}{c}100 \mathrm{~Pa} \sim \\
4 \mathrm{kPa}\end{array}$ & N/A \\
\hline Rigid & $\begin{array}{c}\text { micropillar } \\
\text { structure on } \\
\text { conductive PPy } \\
\text { film }\end{array}$ & Resistive & $\begin{array}{l}\text { PDMS molding } \\
\text { with SI mold }\end{array}$ & $0.03-17$ & $\begin{array}{l}2 \mathrm{~Pa} \sim \\
3 \mathrm{kPa}\end{array}$ & $\sim 1000 \mathrm{~ms}$ \\
\hline $\begin{array}{l}\text { Stretchable } \\
\text { up to } 150 \%\end{array}$ & $\begin{array}{l}\text { Stretched CNT on } \\
\text { PDMS }\end{array}$ & $\begin{array}{c}\text { Capacitive and } \\
\text { Resistive }\end{array}$ & $\begin{array}{c}\text { Spray coating of } \\
\text { CNT, multiple } \\
\text { layer stack }\end{array}$ & $2.3 \times 10^{-6}$ & $50 \mathrm{kPa} \sim$ & $<125 \mathrm{~ms}$ \\
\hline Flexible & $\begin{array}{c}\text { polymer } \\
\text { semiconductor/PD } \\
\text { MS dielectric }\end{array}$ & Capacitive & \begin{tabular}{c|} 
assembling \\
separate layers \\
via lamination
\end{tabular} & $\begin{array}{c}8.4 \\
(<8 \mathrm{kPa})\end{array}$ & $50 \mathrm{~Pa} \sim$ & $<10 \mathrm{~ms}$ \\
\hline $\begin{array}{l}* \text { Flexible } \\
\text { and } \\
\text { Stretchable } \\
\text { up to } 50 \%\end{array}$ & \begin{tabular}{|c|} 
Au coated micro \\
pillar structure on \\
PDMS film
\end{tabular} & Capacitive & $\begin{array}{c}\text { Soft } \\
\text { lithography }\end{array}$ & 0.34 & $\begin{array}{l}80 \mathrm{~Pa} \sim \\
10 \mathrm{kPa}\end{array}$ & 60.8 \\
\hline
\end{tabular}

* The current thesis research results reported here. 


\section{Chapter 5}

\section{Computational Analysis of Micropillar Sensor Arrays}

COMSOL is a multiphysics computational package capable of running coupled electromechanical simulations accounting for finite deformations and electrostatics in 3D. Computational simulations were carried out in COMSOL to investigate how large conformational changes of the pillars during deformation influence the capacitance readings for the different sensor designs. The use of the software is ideal since a host of results can be generated for complex microstructures. The simulations were particularly useful in not just capturing three-dimensional mechanical field effects but also calculating secondary electrical mechanisms such as fringing capacitance, which is rather complicated to calculate using existing mathematical formulations.

In a typical model of a parallel plate capacitor, only the main electric field (taken as uniform between the electrodes) is considered and the capacitance of the structure is computed according to (2.7). This is a simple formula that captures the primary variables influencing a basic parallel-plate capacitor, but is not accurate when the distance between the electrodes is on the same length scale of the electrode area. Previous work has shown that the Palmer formula for fringing field effects has good correlation with FEM simulations for a rectangular parallel plate capacitor. The $2 \mathrm{D}$ Palmer formula is given in (5.1) for completeness, though not explicitly used in this thesis. Comparison of the formula result with simulation work can be found elsewhere [86]. 


$$
\begin{gathered}
\mathrm{C}=\frac{\varepsilon w L}{d}\left(1+\frac{d}{\pi w}\left(1+\ln \left(\frac{2 \pi w}{d}\right)\right)\right) \\
\times\left(1+\frac{d}{\pi L}\left(1+\ln \left(\frac{2 \pi L}{d}\right)\right)\right),
\end{gathered}
$$

where $\mathrm{w}$ and $\mathrm{L}$ are the width and the length of electrodes, $\mathrm{d}$ is the distance between the electrodes, and $\varepsilon$ is the dielectric constant.

The micropillar array can be approximated to first order as a series of parallel plate micro-capacitors with alternating dielectric media of PDMS+air and PDMS only. Even then such an equivalent circuit would grossly overlook the effect of the nonplanar electrodes. Since the bottom electrode conforms to the pillar geometry, the problem is more complicated i.e. the electrode itself is microstructured. This is the source of additional nonuniformity of the electric field. The capacitance of the structure including the fringing field can be accurately computed using finite element methods (FEM). Figure 5.1 outlines the problem formulation and Figure 5.2 illustrates the nonuniformity of the field as well as the fringe field.

Mechanical B.C.1: Top and side surface of top film; fixed in $\mathrm{x}, \mathrm{y}$ directions

Electrical B.C.1: Top gold layer as terminal, $1 \mathrm{~V}$ potential applied

Electrical B.C.2: Bottom gold layer as ground

Mechanical loading: uniform pressure applied on top rigid plate

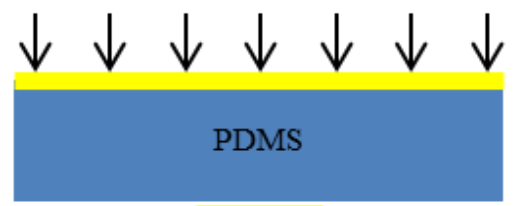

Air

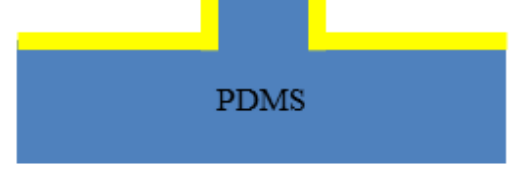

Mechanical B.C.2: Bottom surface of bottom film; fixed in 3 directions
Film dimensions Gold: $1 \mu \mathrm{m}$ thickness PDMS: $100 \mu \mathrm{m}$ thickness

Contact between top and bottom films -Comsol: contact pair assigned on bottom surface of top PDMS and top surface of bottom gold electrode -Abaqus: Two parts are assembled as one part

Figure 5.1 Problem formulation in ABAQUS and COMSOL. 
The fringing field significantly increases the capacitance of the system. Furthermore, as will be shown in the results, the fringe field effect is responsible for the increase in sensitivity perceived in the sensor results. The fringe field effect is most dominant during the initial loading period and converges to the simplified formula result when the gap between electrodes narrows. When the electrodes are closer together the extra capacitance decreases due to the attenuating fringing field.

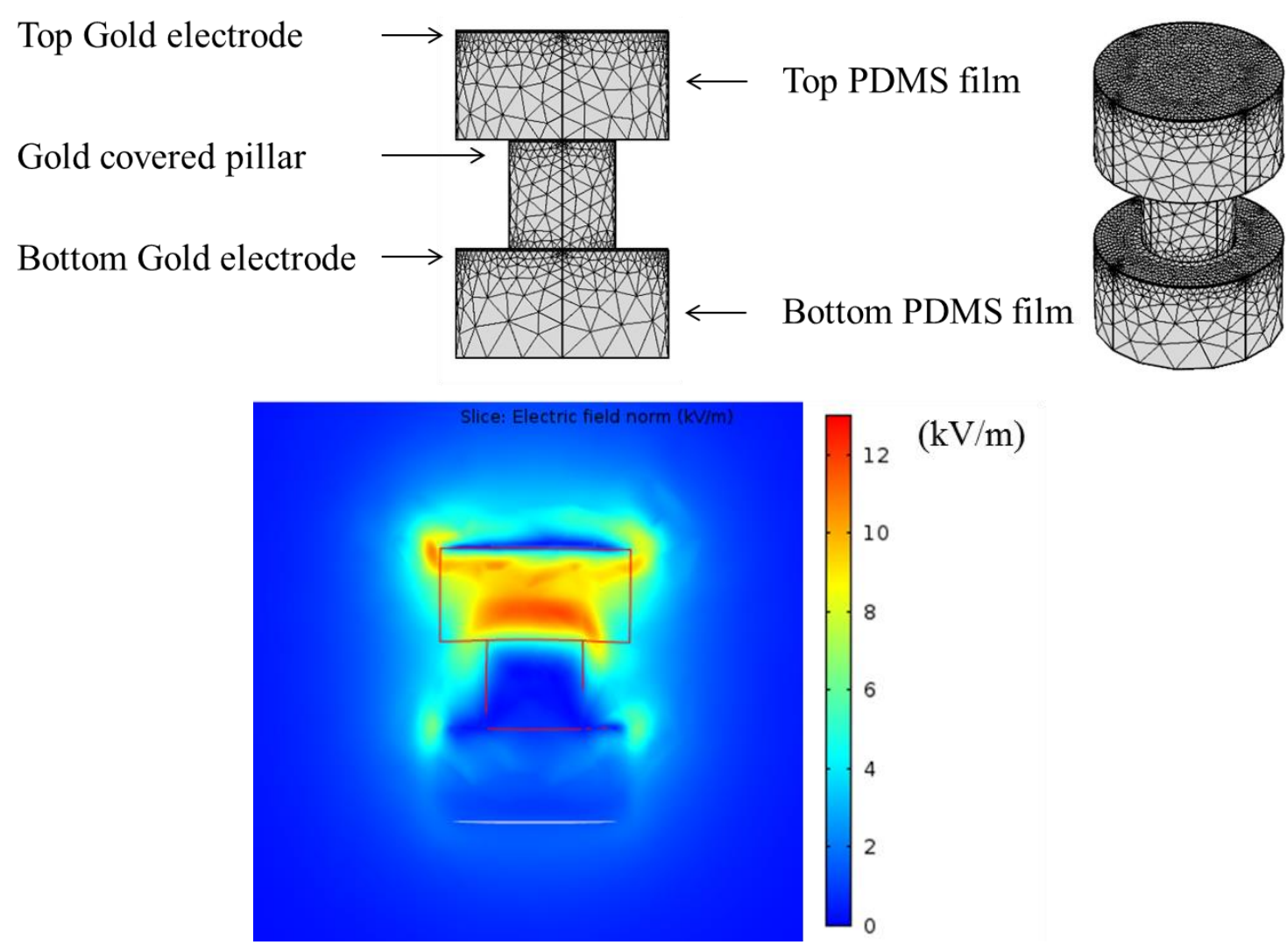

Figure 5.2 The nonuniform electric field due to spatially varying permittivity and fringe field effects of a single pillar. The electric field is overlaid on the deformed pillar geometry. Note that the bulk of the field is contained in the PDMS monolithic layer.

\subsection{Setup of the problem}

PDMS micropillar arrays (or single pillars where accurate) were generated of varying geometric designs. A $1 \mu \mathrm{m}$ gold layer is placed on top of the PDMS array. A monolithic 
PDMS film then rests on top of the array and is covered with a monolithic AU film. Air fills the gaps between the pillars. The properties of the layers are specified in Table 5.1.

Table 5.1 Material properties for COMSOL simulations.

\begin{tabular}{|c|c|c|c|}
\hline Material Property & PDMS & Gold layer & Air \\
\hline Young's modulus & N/A & $60 \mathrm{GPa}$ & N/A \\
\hline Relative permittivity & 2.5 & 6.9 & 1.00059 \\
\hline Poisson's ratio & 0.499 & 0.42 & N/A \\
\hline Density & $0.97 \mathrm{~kg} / \mathrm{m} 3$ & $19300 \mathrm{~kg} / \mathrm{m} 3$ & N/A \\
\hline $\begin{array}{c}\text { Mooney Rivilin } \\
\text { (Hyperelastic) }\end{array}$ & $\begin{array}{c}\mathrm{C} 10=73.35 \mathrm{kPa} \\
\mathrm{C} 01=5.7 \mathrm{kPa} \\
\text { K-7.7 } \mathrm{GPa}\end{array}$ & N/A & N/A \\
\hline
\end{tabular}

All design geometries are generated in COMSOL and the initial and final capacitances calculated. The mechanical part of the COMSOL simulation was compared with a simulation in ABAQUS as a check. A single pillar of circular geometry with a 1:1 ratio $(100 \mu \mathrm{m} \times 100 \mu \mathrm{m})$ was subjected to a pressure load $(9 \mathrm{kPa})$. Figure 5.3 shows a comparison of the stress and displacement fields in the thickness direction and the overall displacement of a single pillar and the results clearly match well.

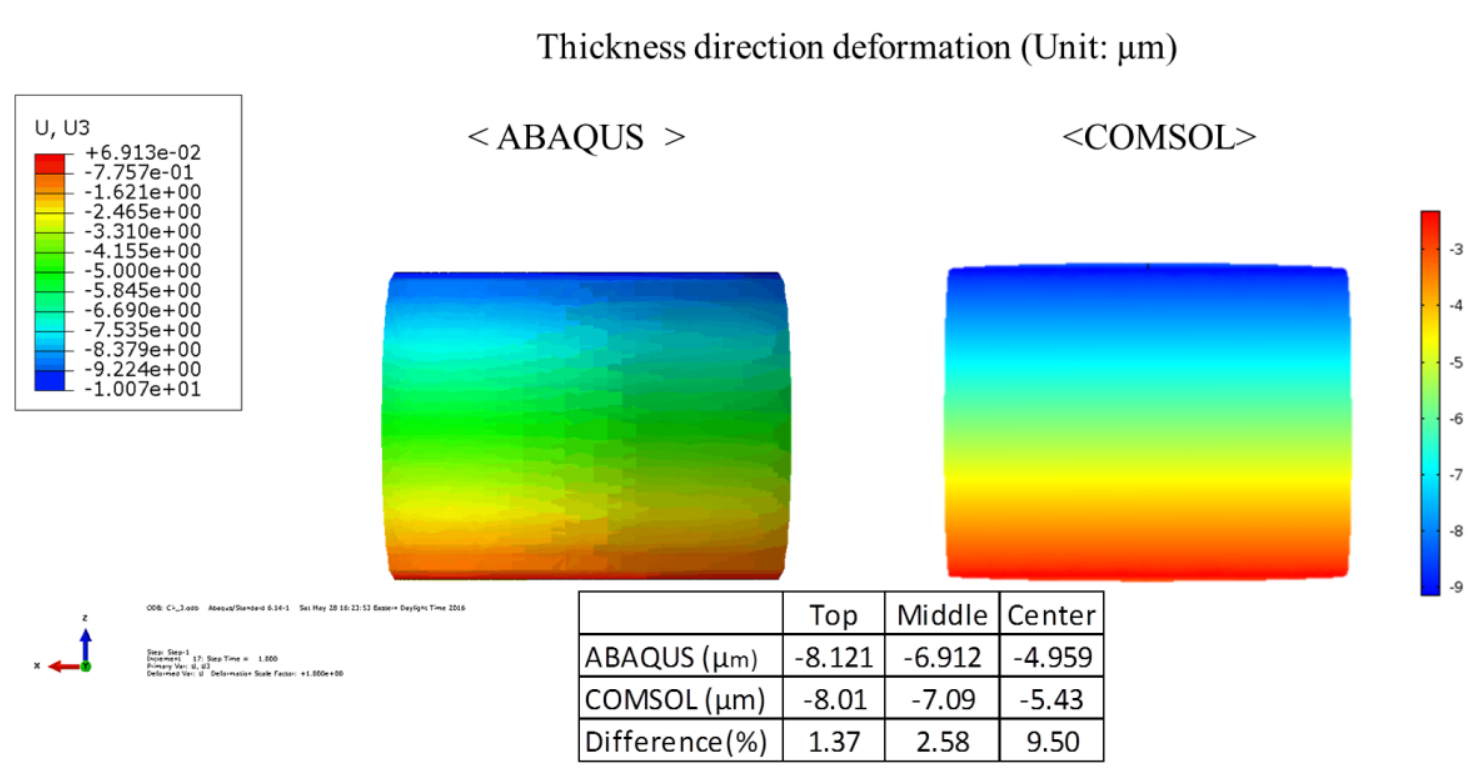


Thickness direction principal stress on slide (Unit: $\mathrm{kPa}$ )
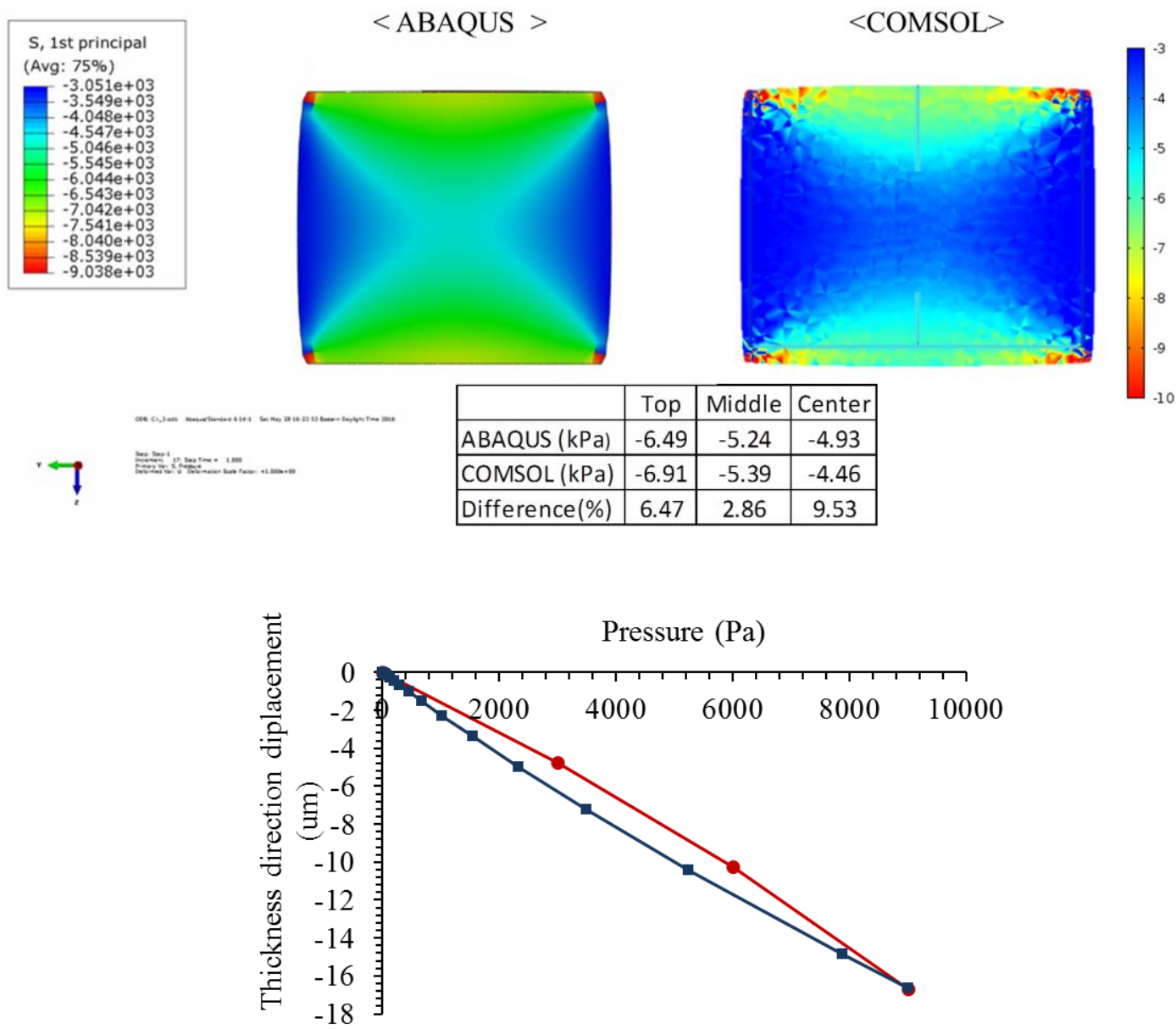

Figure 5.3 Comparison of the stress and displacement fields in the thickness direction and the overall displacement of a single pillar from ABAQUS and COMSOL.

\subsection{Coupled electromechanical simulations}

\subsubsection{Electrostatic field}

For the electrostatics part in COMSOL, the surrounding domain of air volume must be sufficiently large to ensure that the fringing field is accurately calculated, and appropriate boundary conditions must be specified. The domain size was varied while maintaining the mesh size to study convergence and ensure stability of the capacitance result for the boundary conditions selected. Figure 5.4 shows electric field for varying domain sizes. 
This test is analogous to the standard mesh convergence test done for mechanical results in FEM. Note that the dielectric layer needs to be 3-5 elements thick to sufficiently resolve the electric field.

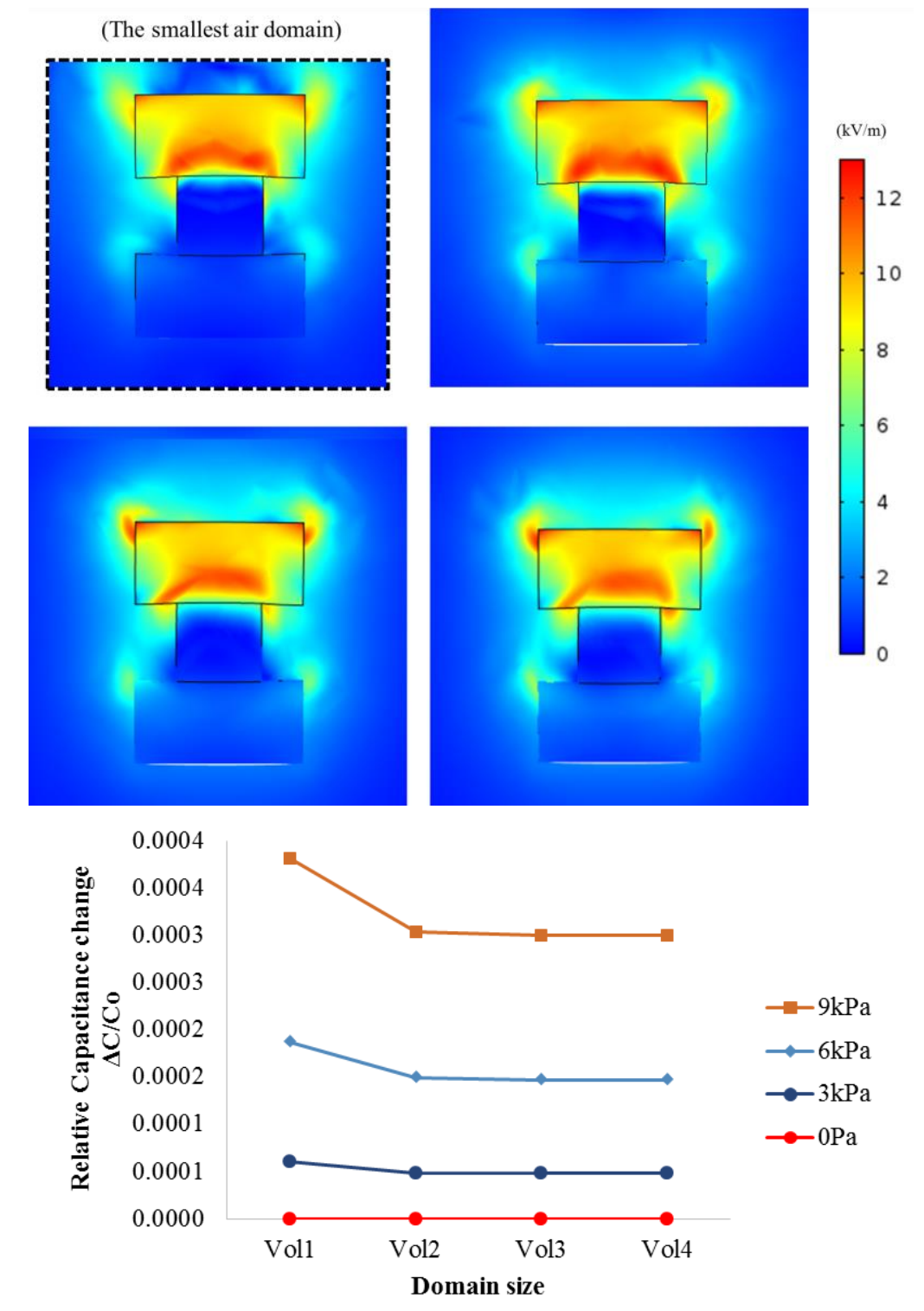

Figure 5.4 Convergence test for capacitance calculation performed by studying the effect of air domain size on the fringe field whilst keeping the mesh constant. Plot of relative capacitance as a function of domain size for different levels of loading. 


\subsubsection{COUPLED SIMULATIONS}

In order to design the pillar and sensor device in COMSOL (and ABAQUS for comparison of the mechanical part), the electrostatics (es) module and the electromechanics (emi) module were run in coupled fashion. The mechanical part calculates the deformed shape and distribution of stress. The electrical part outputs the initial capacitance of the sensor assembly, and the final capacitance of the assembly post deformation (due to a $9 \mathrm{kPa}$ load). For ease of comparison, several design metrics were kept the same in order to focus on a subset of key parameters. The simulation test matrix is given in Table 5.2 and Figure 5.5 shows schematics of the different cross-sections used in the simulations.

Table 5.2 Test matrix for COMSOL simulations.

\begin{tabular}{|c|c|c|c|c|c|}
\hline \multirow{2}{*}{$\begin{array}{c}\text { Sample } \\
\#\end{array}$} & \multirow{2}{*}{$\begin{array}{l}\text { Cross } \\
\text { section } \\
\text { shape }\end{array}$} & \multirow{2}{*}{$\begin{array}{c}\text { Aspect } \\
\text { ratio }\end{array}$} & \multicolumn{3}{|c|}{ Dimension $(\mu \mathrm{m})$} \\
\hline & & & gap & height & width \\
\hline 1 & \multirow{6}{*}{ Square } & 1:1 & 100 & 100 & 100 \\
\hline 2 & & $1: 3$ & 100 & 300 & 100 \\
\hline 3 & & $1: 5$ & 100 & 500 & 100 \\
\hline 4 & & $1: 7$ & 100 & 700 & 100 \\
\hline 5 & & $1: 1$ & 200 & 100 & 100 \\
\hline 6 & & $1: 1$ & 300 & 100 & 100 \\
\hline 12 & Circle & $1: 1$ & 87.2 & 100 & 112.8 \\
\hline
\end{tabular}

\begin{tabular}{|c|c|c|c|c|c|c|}
\hline $\begin{array}{c}\text { Sample } \\
\#\end{array}$ & $\begin{array}{c}\text { Cross section } \\
\text { shape }\end{array}$ & OR:IR & IR & OR & gap & height \\
\hline 13 & $\begin{array}{c}\text { Half Circle } \\
\text { (Easy to } \\
\text { buckle) }\end{array}$ & $5: 3$ & 42.3 & 70.5 & 100 & 100 \\
\cline { 3 - 7 } 14 & $5: 4$ & 75.2 & 94.0 & 100 & 100 \\
\hline
\end{tabular}

\begin{tabular}{|c|c|c|c|c|c|c|}
\hline \multirow{2}{*}{$\begin{array}{c}\text { Sample } \\
\#\end{array}$} & \multirow{2}{*}{$\begin{array}{c}\text { Cross } \\
\text { section } \\
\text { shape }\end{array}$} & \multirow{2}{*}{$\begin{array}{l}\text { Aspect } \\
\text { ratio }\end{array}$} & \multicolumn{3}{|c|}{ Dimension $(\mu \mathrm{m})$} & \multirow{2}{*}{ Array } \\
\hline & & & gap & height & width & \\
\hline 15 & \multirow{4}{*}{ Square } & \multirow{4}{*}{$1: 1$} & \multirow{4}{*}{100} & \multirow{4}{*}{100} & \multirow{4}{*}{100} & 1 \\
\hline 16 & & & & & & $10 \times 10$ \\
\hline 17 & & & & & & $25 \times 25$ \\
\hline 18 & & & & & & $50 \times 50$ \\
\hline
\end{tabular}




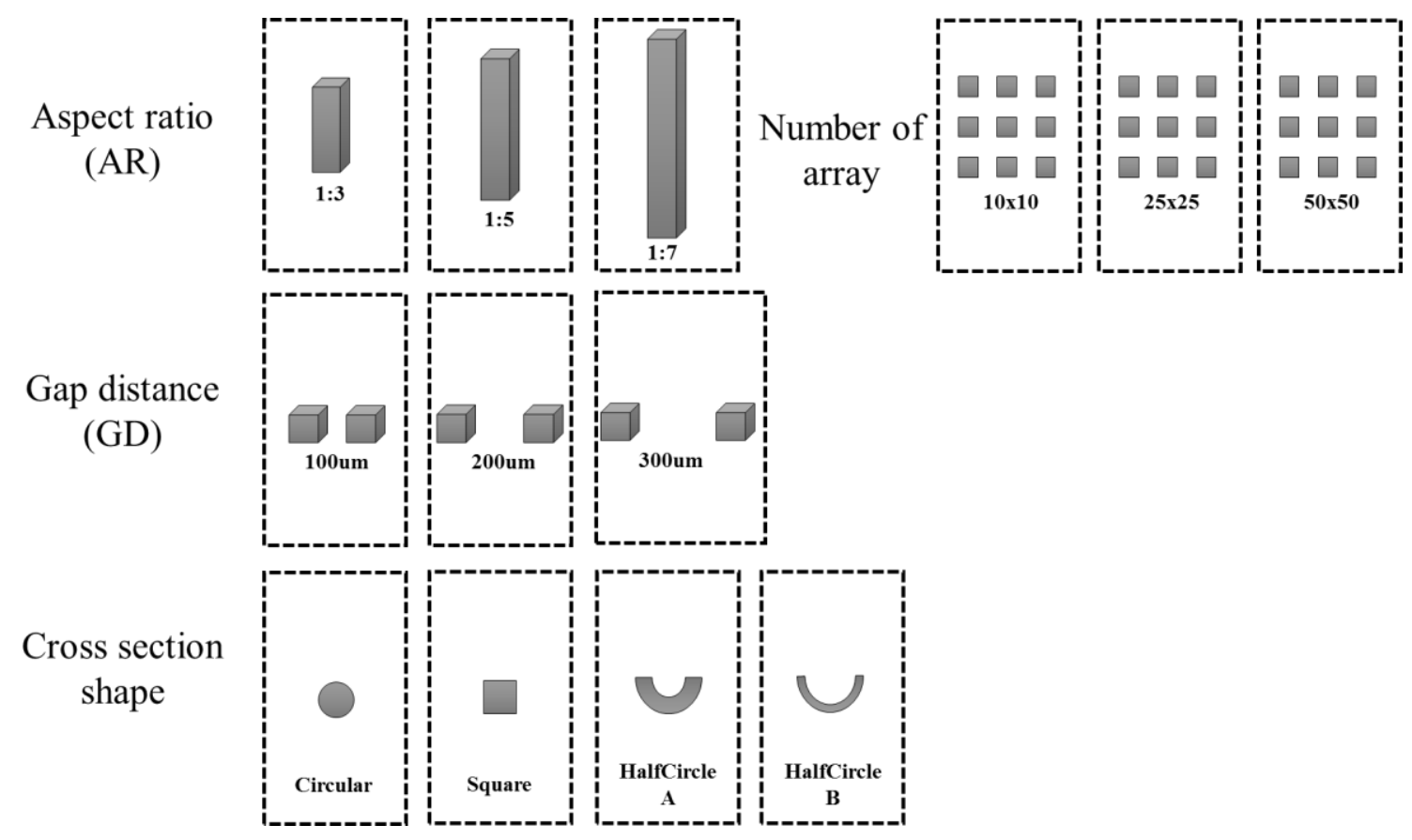

Figure 5.5 Geometrical aspects of the pillar designs simulated and tested.

\subsection{Sensor results}

In the experimental results, arrays of $50 \times 50$ pillars were fabricated and tested. To simulate the mechanical response of the pillar for the different designs, a single pillar was found to capture the overall deformation quite well except for large gap distances between the pillars in which case there is notable sagging of the top monolithic film in between the pillars. Electrically, the number of pillars influences the capacitance reading since the electrode area varies. Figure 5.6 shows the effect of increasing the number of pillars on the relative capacitance. As the results show, there is a very good match between the simulation and experimental results for the 50 x 50 array. Increasing the number of pillars in the arrays increases the computation run time of the analysis. To investigate the influence of sensor design on performance, the analyses were conducted for an array size of $25 \times 25$ to save on computational cost. The simulation results can be compared to each other and are qualitatively useful to probe the trends observed in the experimental results. It should be noted that for all the simulations the pillars at the four corners are fixed in the $x-y$ plane to stabilize the array and ensure fast computational convergence. 

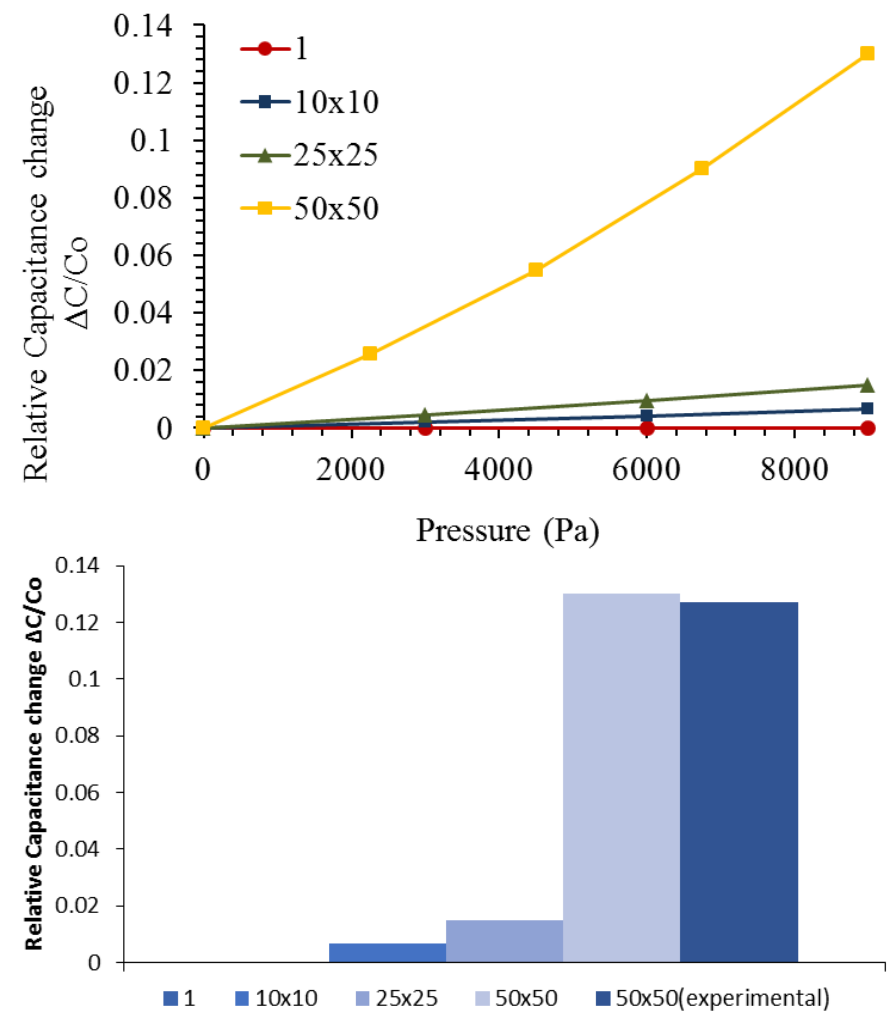

Figure 5.6 Increasing the number of pillars increases the sensitivity. There is a very good match with experimental results when the entire array is replicated.

Figure 5.7 summarizes the calculated sensor output for varying aspect ratio, cross-section shape, and gap distance. The results show identical trends to the experimental results. Specifically, increasing the aspect ratio and pillar geometries designed to buckle dramatically increase sensor sensitivity. Increasing gap distance can also improve sensitivity. To a smaller extent, pillar cross-section (for constant area) can influence the sensitivity as well. A simple Euler-Bernoulli beam analysis shows that circular cross section beams bend more easily in comparison to square ones. Similarly, the Euler buckling load for axially loaded columns shows that circular cross-section columns buckle more easily in comparison to square cross-section ones. Therefore, the increased deformation for a given load more readily narrows the electrode gap and causes the slight increase in sensitivity for circular pillars. It should be noted that because of the limited 
number of data points, regime specific variations that appear in the experimental results are not clearly captured in the simulation output. Sensitivity results for the different simulations are shown in Table 5.3.
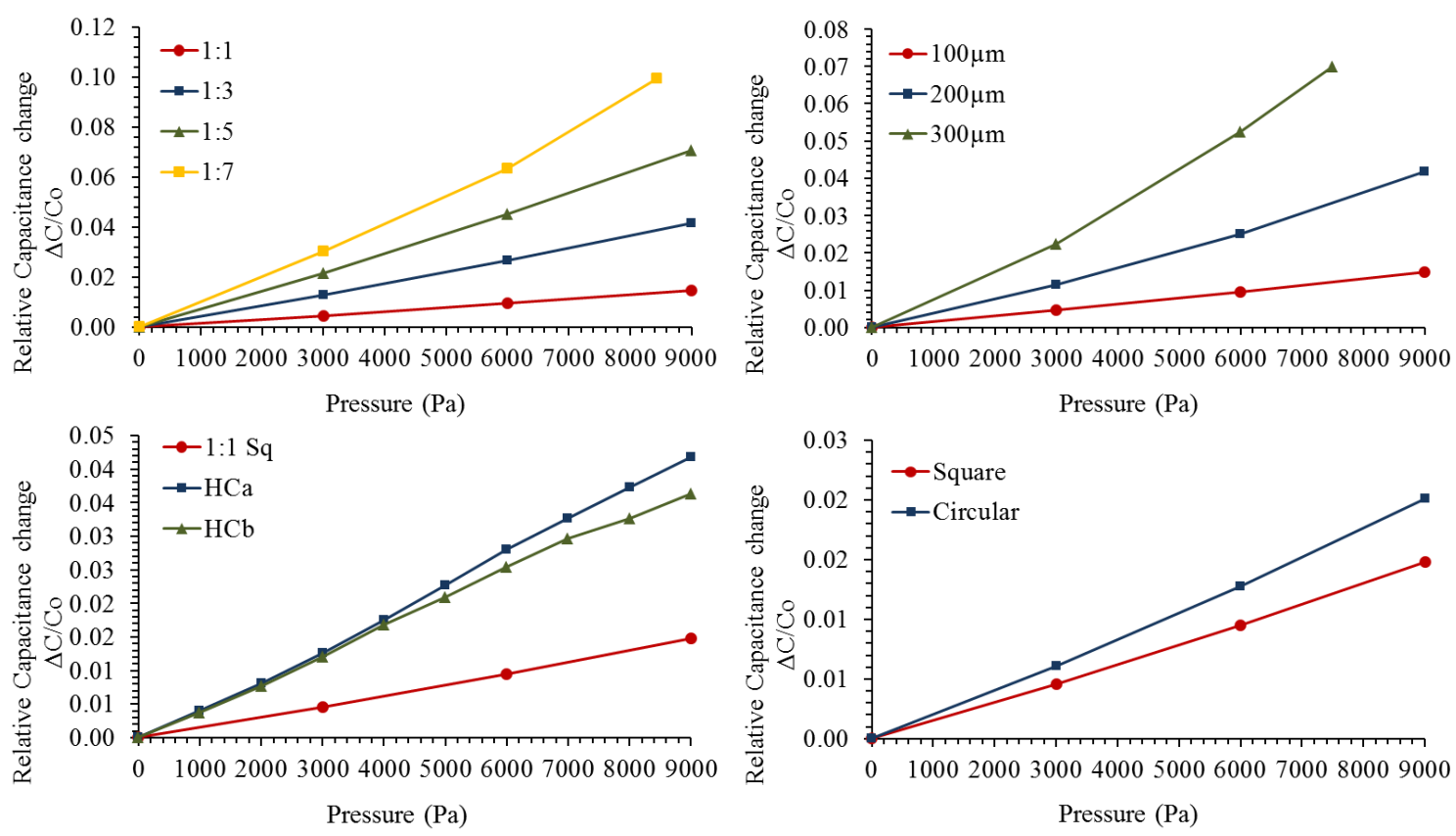

Figure 5.7 Computational results for sensor output for the different designs.

Table 5.3 Sensitivity results for the different array designs (simulation results).

\begin{tabular}{|c|c|}
\hline Aspect ratio & $\begin{array}{c}\text { Sensitivity from } \\
1 \mathrm{~Pa}-10 \mathrm{kPa}\left(\mathrm{kPa}^{-1}\right)\end{array}$ \\
\hline $1: 1$ & 0.02 \\
\hline $1: 3$ & 0.05 \\
\hline $1: 5$ & 0.08 \\
\hline $1: 7$ & 0.1 \\
\hline $100 \mu \mathrm{m}$ gap & 0.02 \\
\hline $200 \mu \mathrm{m}$ gap & 0.05 \\
\hline $300 \mu \mathrm{m}$ gap & 0.09 \\
\hline $\mathrm{HCa}$ & 0.05 \\
\hline $\mathrm{HCb}$ & 0.04 \\
\hline Circular & 0.02 \\
\hline
\end{tabular}


Detailed views of pillar deformation and electric fields provides some insight as to the source of improved sensitivity experimentally observed. The mechanical and electric fields for different pillar array sizes (single pillar, 10 x 10 array, 25 x 25 array, and 50 x 50 array) are shown in Figures 5.8-5.11. Analysis of the $25 \times 25$ pillar result shows that as expected the bulk of the deformation occurs within the pillar, strains are highest there. The level of strain is on the order of $10 \%$ for a $9 \mathrm{kPa}$ pressure load.

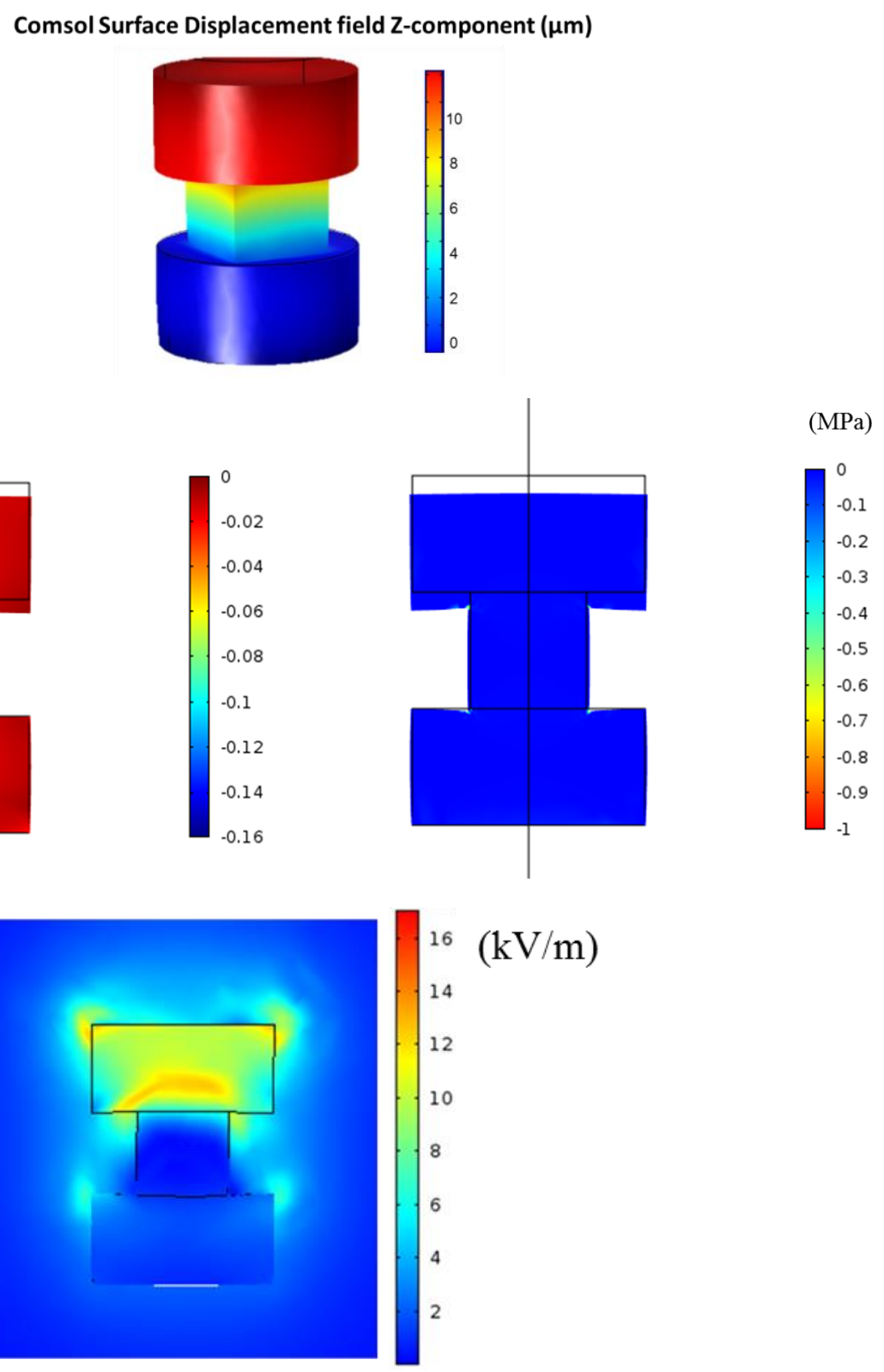

Figure 5.8 Overall geometry and strain of single pillar (top) mechanical stress and strain fields (middle) and electric field distribution (bottom). 

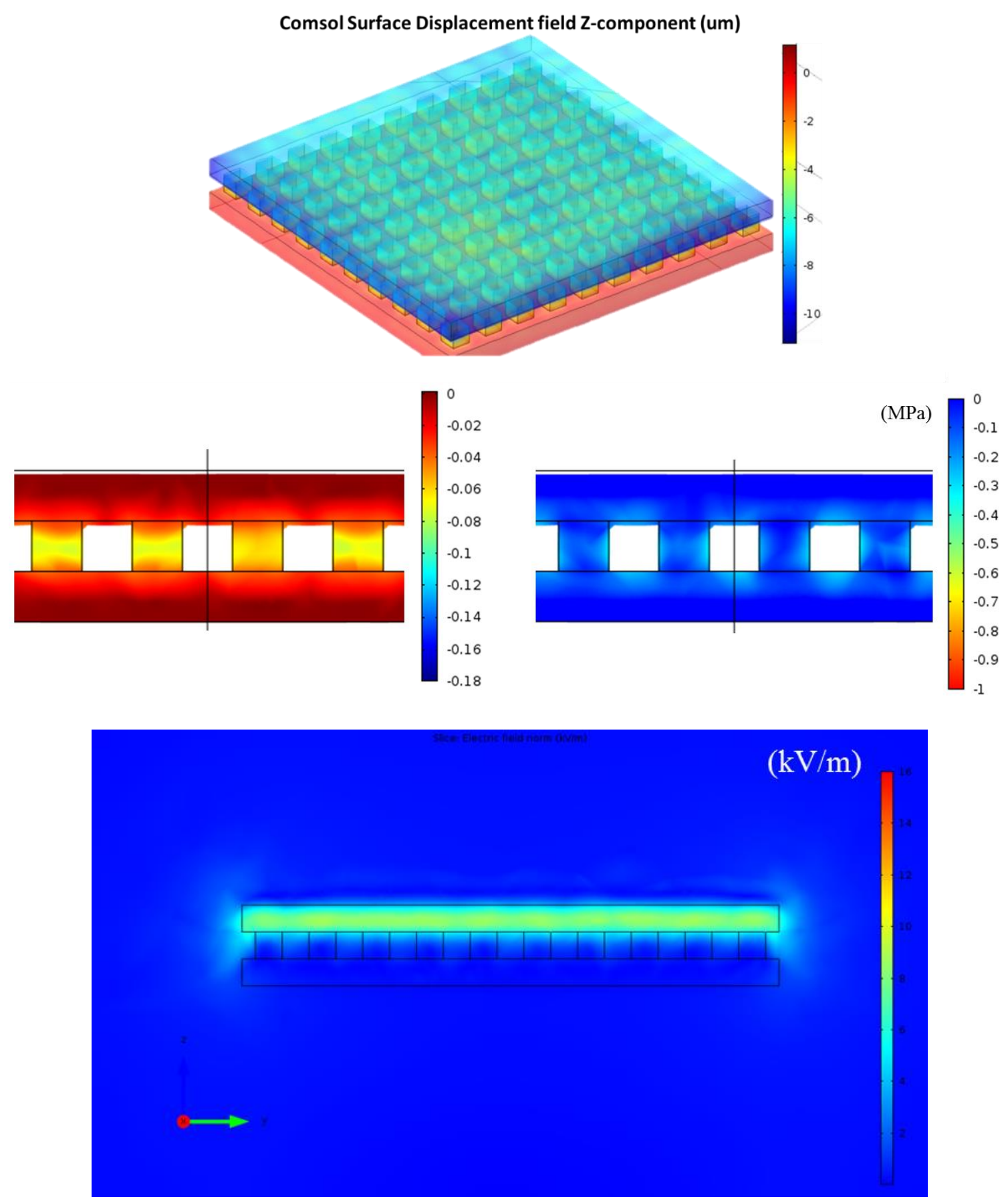

Figure 5.9 Overall geometry and displacement of $10 \times 10$ array (top) mechanical stress and strain fields (middle) and electric field distribution (bottom). 

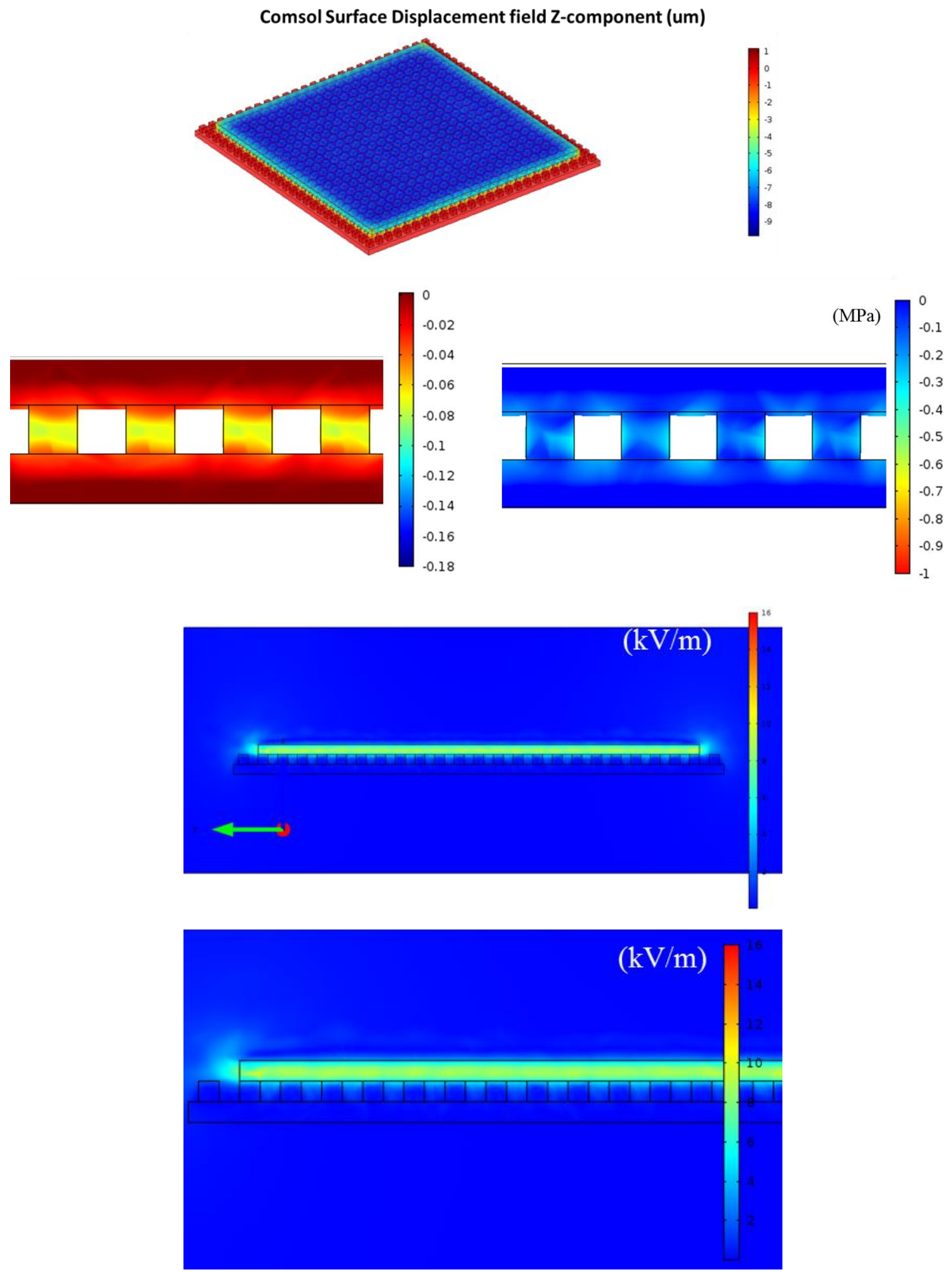

Figure 5.10 Overall geometry and displacement of $25 \times 25$ array (top) mechanical stress and strain fields (middle) and electric field distribution with zoomed in view(bottom). 


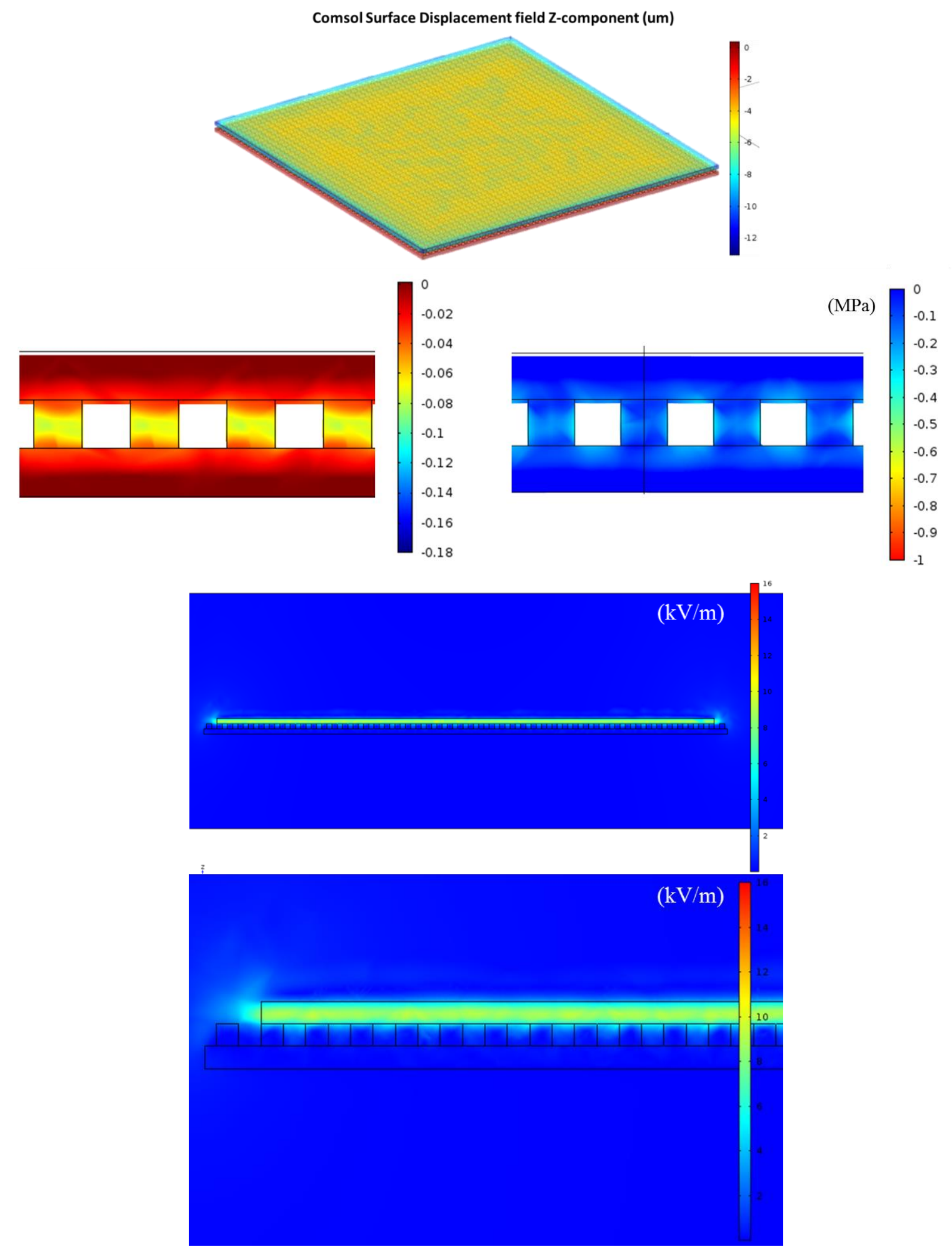

Figure 5.11 Geometry of 50 x 50 array (top) mechanical stress and strain fields (middle) and electric field distribution with zoomed in view (bottom). 
Figure 5.12 provides a side by side comparison of the electric field as a function of array size. The nonuniformity of the electric field and the electric fringe field are clearly defined. The electric field is mostly contained in the monolithic PDMS layer and has a magnitude of approximately $10 \mathrm{kV} / \mathrm{m}$. The field also partially extends down the pillar length. The fringe field is clearly visible at the edge and above the top electrode.

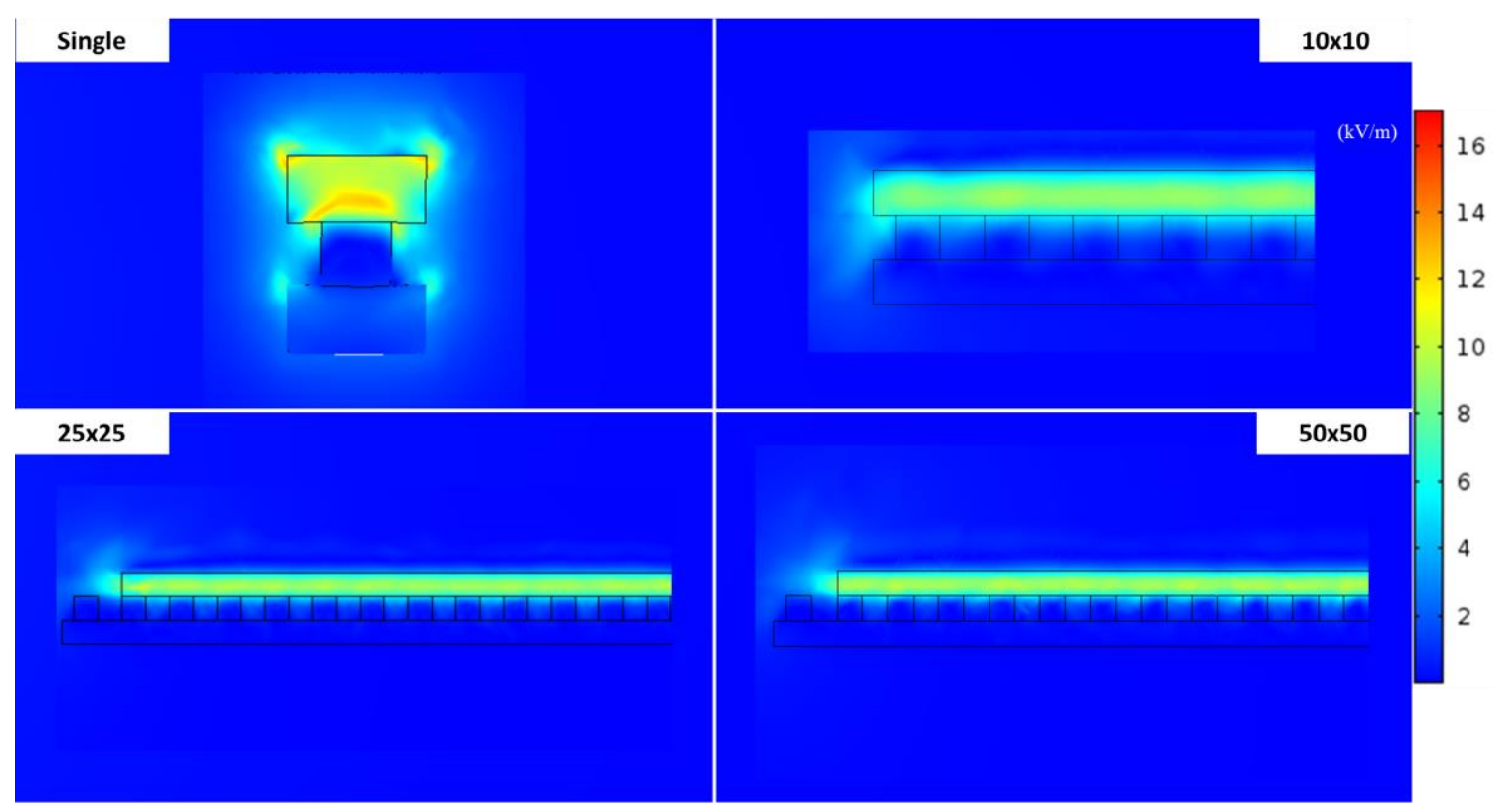

Figure 5.12 Zoomed in views of electric field as a function of the number of pillars in the simulated array.

Figures 5.13 show the field results for micropillar arrays of varying aspect ratios 1:1, 1:3, 1:5, and 1:7. The pillars have square cross-sections of $100 \mu \mathrm{m} \times 100 \mu \mathrm{m}$. As aspect ratio increases, there is some sagging of the monolithic film between the pillars and the pillars dramatically bend and buckle. This decreases the gap between the top electrode and the bottom microstructured electrode and alters the shape of the dielectric leading to changes in the instantaneous electric field. These deformation induced changes in the electric field increase the capacitance. The pillars collapse more easily and undergo large conformational changes with increasing aspect ratio, thus sensitivity increases. 


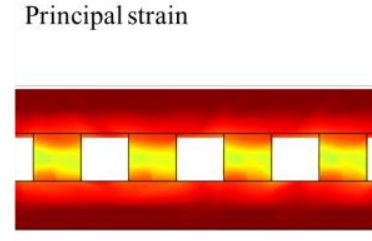

$1: 1$

Principal stress

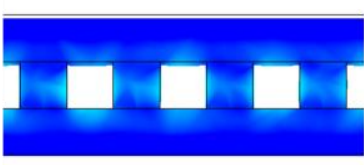

$1: 1$

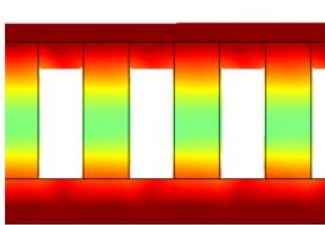

$1: 3$

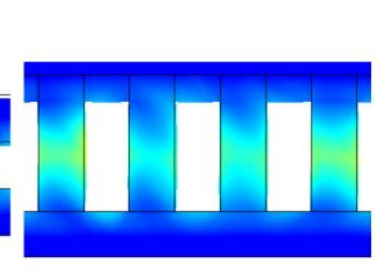

$1: 3$

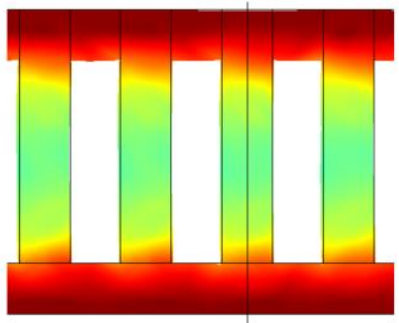

1:5

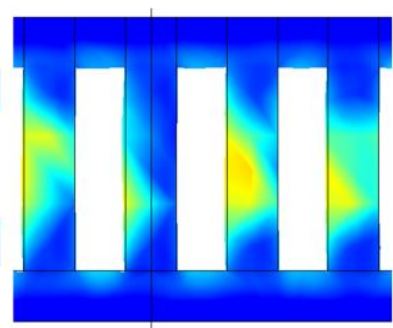

$1: 5$

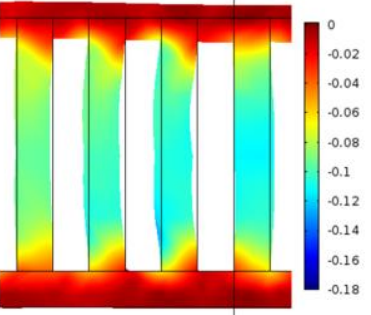

$1: 7$

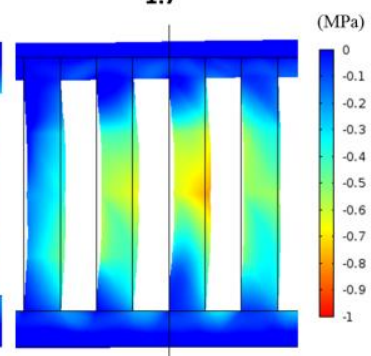

$1: 7$

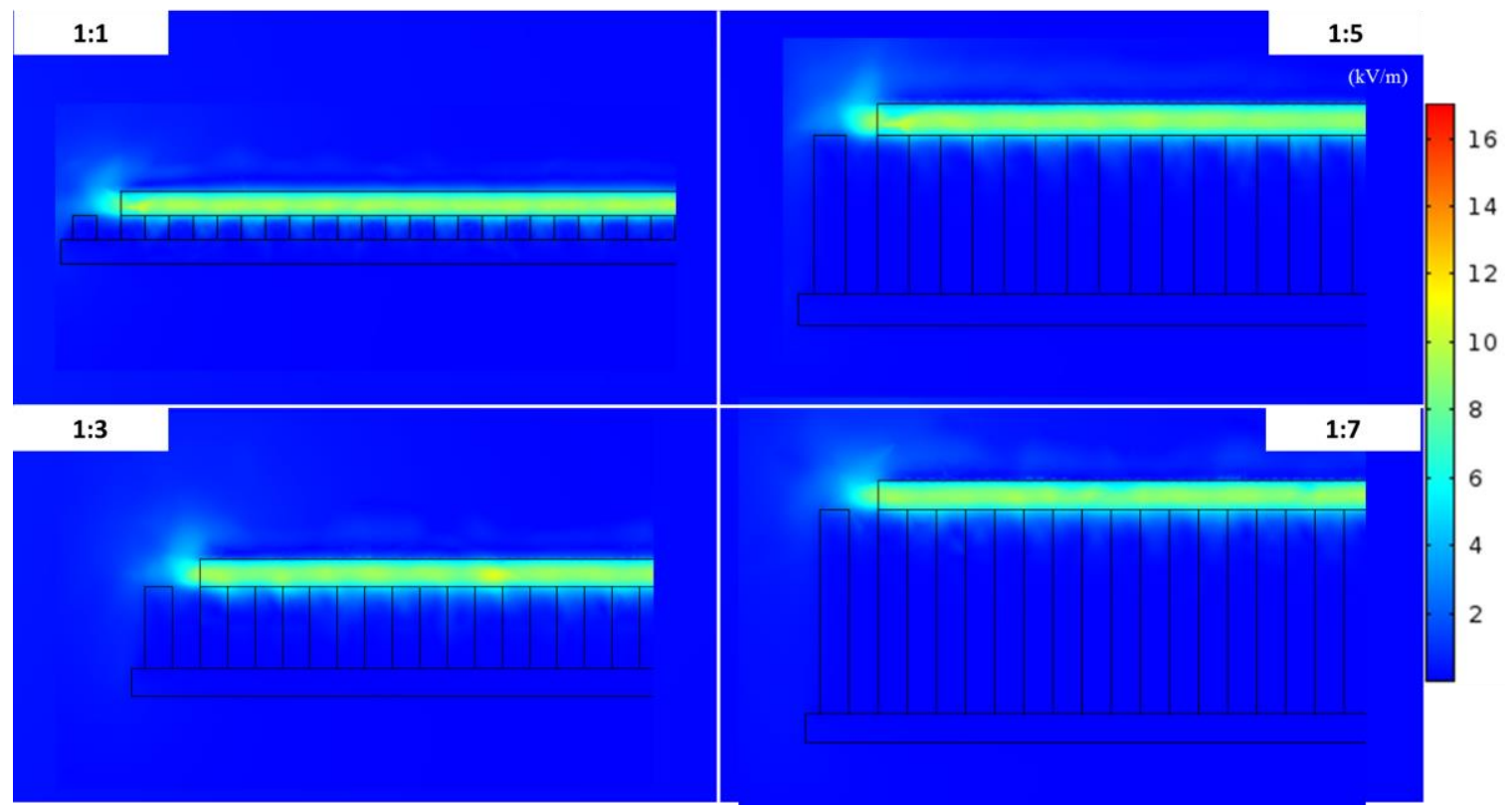

Figure 5.13 Simulation results for the $25 \times 25$ array with varying aspect ratios. Mechanical stress and strain fields (top) and electric field distribution with zoomed in view (bottom).

Figures 5.14 show the field results for micropillar arrays of varying gap distances. The pillars have square cross-sections of $100 \mu \mathrm{m}$ and a 1:1 aspect ratio. The sagging between the pillars is dramatic and this configuration has the largest overall deformation leading to high sensor sensitivities. The evolving nonuniformity of the electric field is both a reflection of large pillar deformations and film deformations, which create a spatially 
varying dielectric medium. At the deformed gap distances, the electric field increasingly permeates the air gap due to proximity of the film to the side and bottom electrode surfaces.

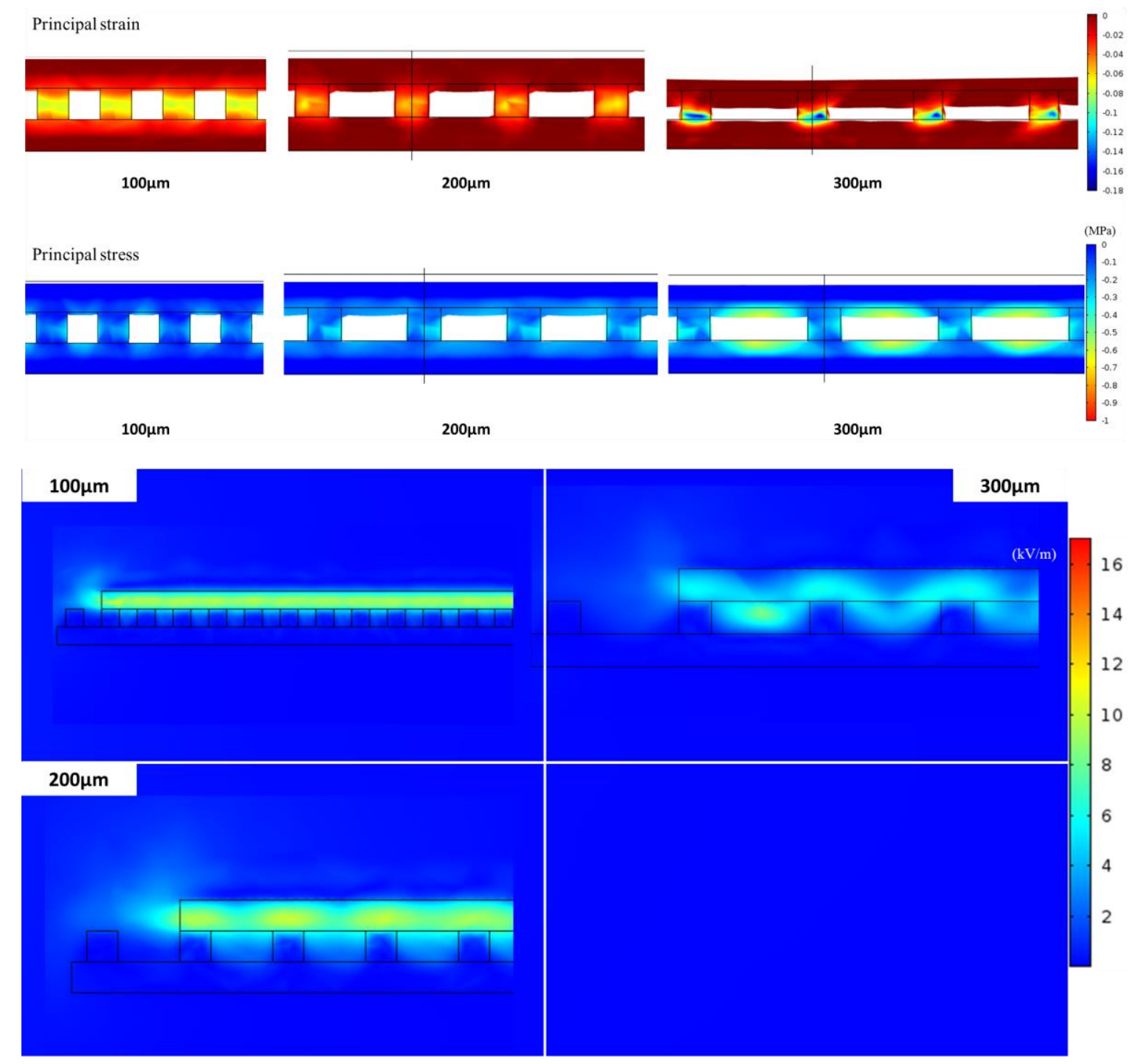

Figure 5.14 $25 \times 25$ array with varying gap distances. Mechanical stress and strain fields (top) and electric field distribution with zoomed in view (bottom).

Field results for two easy to buckle half-circle designs compared to the square pillar design are presented in Figure 5.15. Half circle pillar design B deforms more than design A, which is to be expected. A first order buckling analysis for a thin walled cylinder shows that the buckling load scales with the ratio of $t / R$ (thickness/radius). The deformed 
electric field intensifies near the top of the pillar because of the asymmetric curved electrode configuration distributed over the narrow wall thickness.
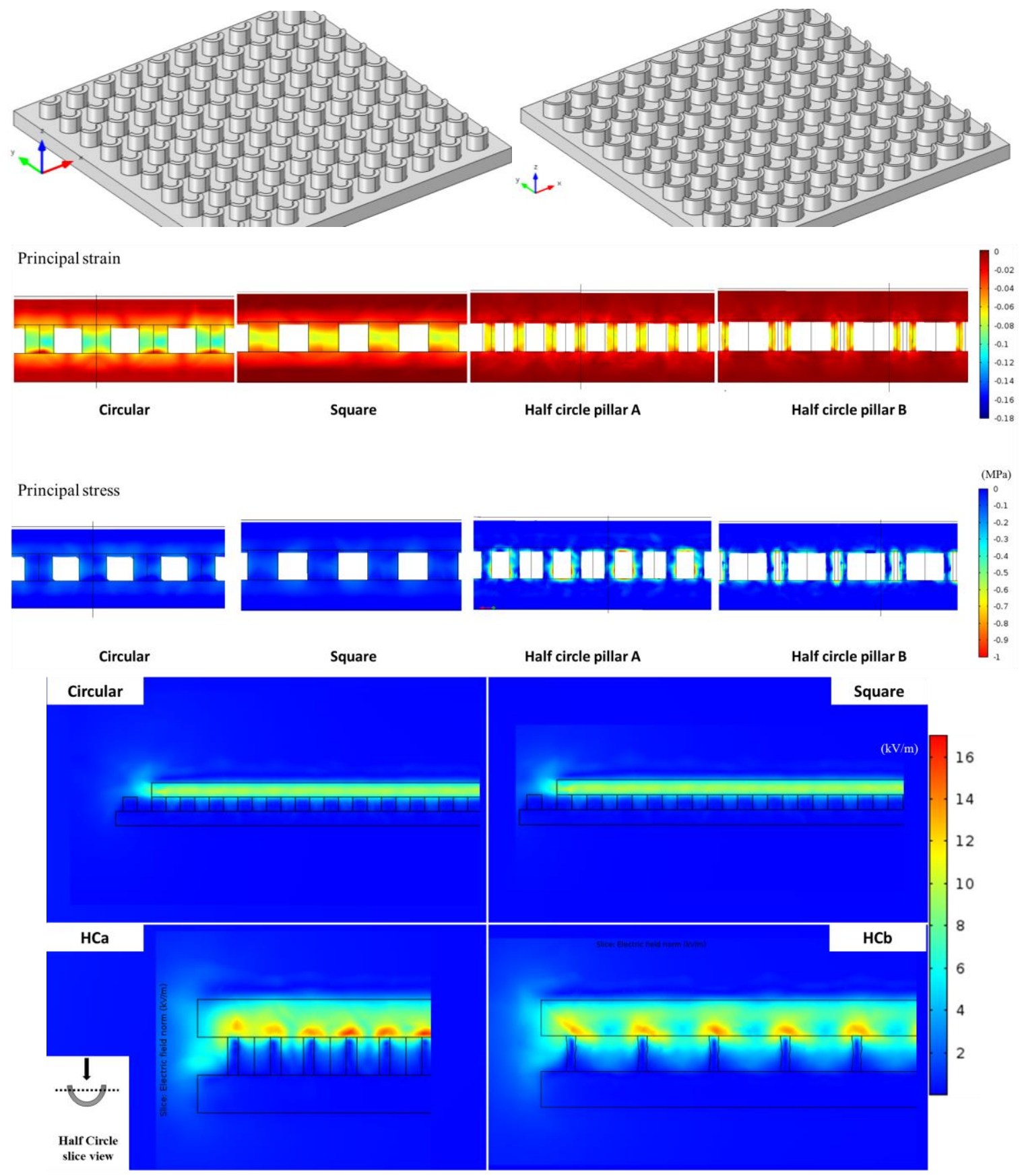

Figure 5.15 Simulation results for a $25 \times 25$ array with 3 varying cross-sections, full circle, thickwalled half circle, and thin-walled half circle compared to a square 1:1 array. Half circle pillar array geometry (top). Mechanical stress and strain fields (middle) and electric field distribution with zoomed in view (bottom). 
Collectively, the results clearly show how the reconfiguration of the dielectric media and electrode surfaces can lead to alterations in the nonuniform electric field. These 3 dimensional field alterations cause large changes in capacitance, and by extension increased sensitivity and pressure sensing range. It is evidenced how pillar geometry can be tailored to optimize sensor performance.

\subsection{Fringe field effect}

The simulations presented in the previous subsection were generated for a uniformly applied pressure in the COMSOL software. Preliminary experimental results showed increased sensitivity in the ultra low-pressure regime 5-100 $\mathrm{Pa}$. To provide some insight as to the origins, a computational approach is used to probe secondary capacitive effects i.e. the effect of the fringing field is examined in detail. The idea is to try to isolate the capacitance change due to proximity from the capacitance change due to applied load. Specifically, we look at the change in capacitance of the sensor array as a function of: i) a massless object (dielectric or conducting) brought in close proximity of the surface but not touching. and ii) a massless object (dielectric or conducting) brought into contact with the surface. There were a total of 6 cases. The object has a $5 \mathrm{~mm}$ diameter and $4 \mathrm{~mm}$ height. Figure 5.16 shows the meshed configuration for the proximity test. Simulation results of the electric field for a dielectric object are presented in Figure 5.18. Figure 5.17 shows the simulation results for a conducting object. The top electrode has $1 \mathrm{~V}$ applied and the bottom microstructured electrode is set to ground. The results show how the object increases the fringe field on top of the sensor surface. Furthermore, if the object is a dielectric, the field in the monolithic PDMS layer is significantly reduced. There is less of an impact for a conducting object. Note that because the simulations are performed in the ESI module and not the AC/DC module in the electromagnetics package, current does not flow and inductive effects are not considered. This is reasonable for the low fields and low frequencies prescribed in the experimental setup. 


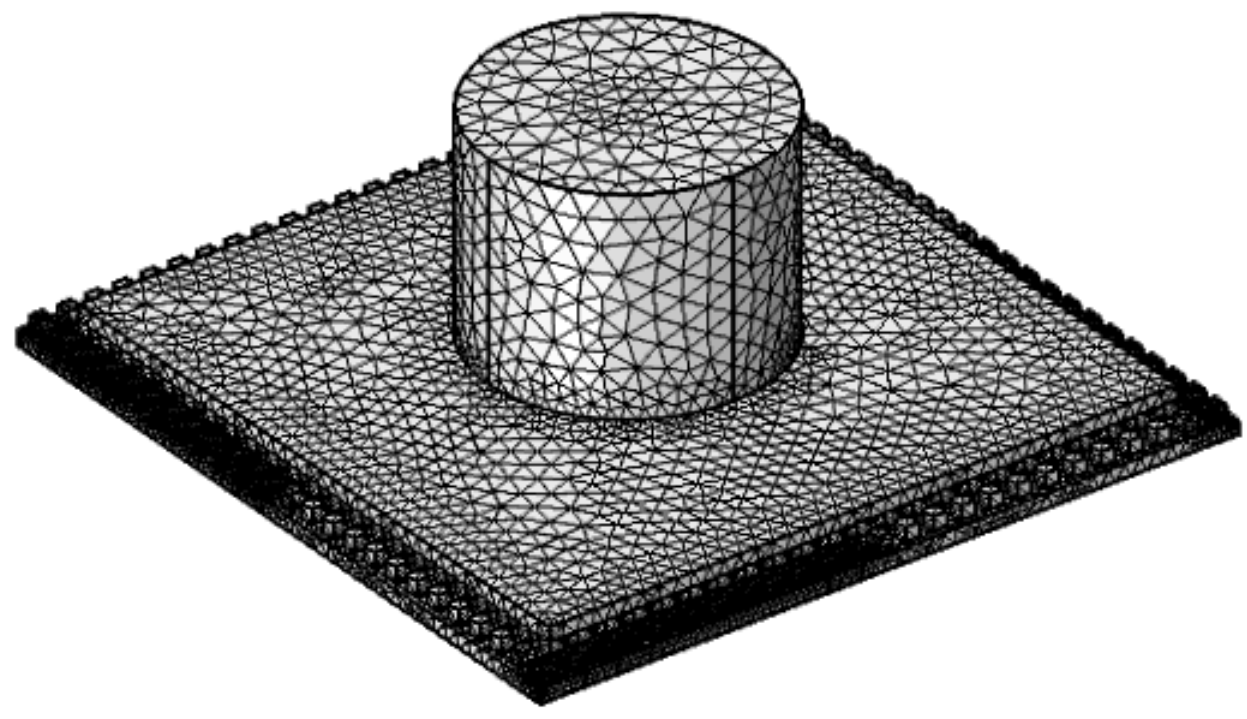

Figure 5.16 Meshed configuration for the proximity test.
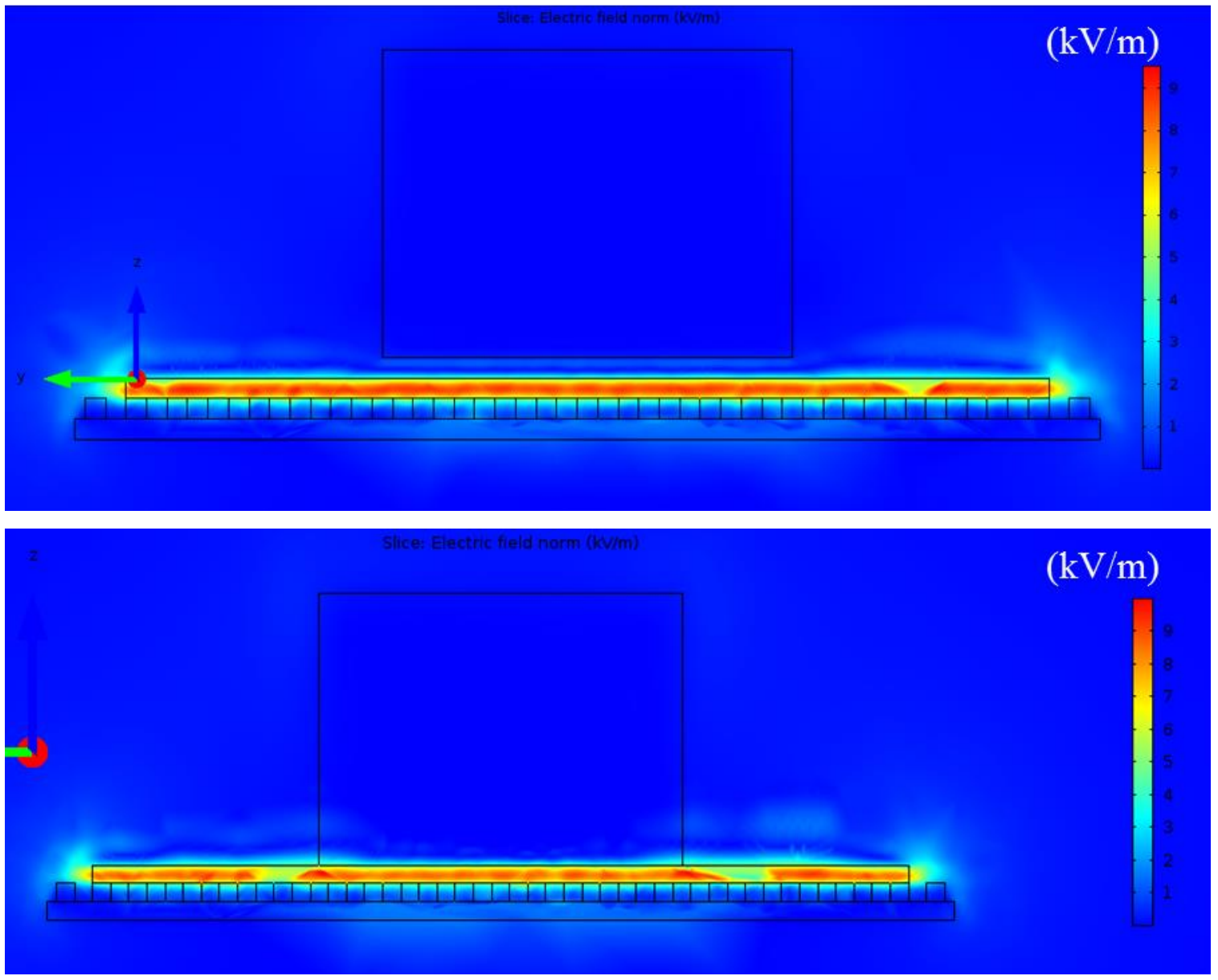

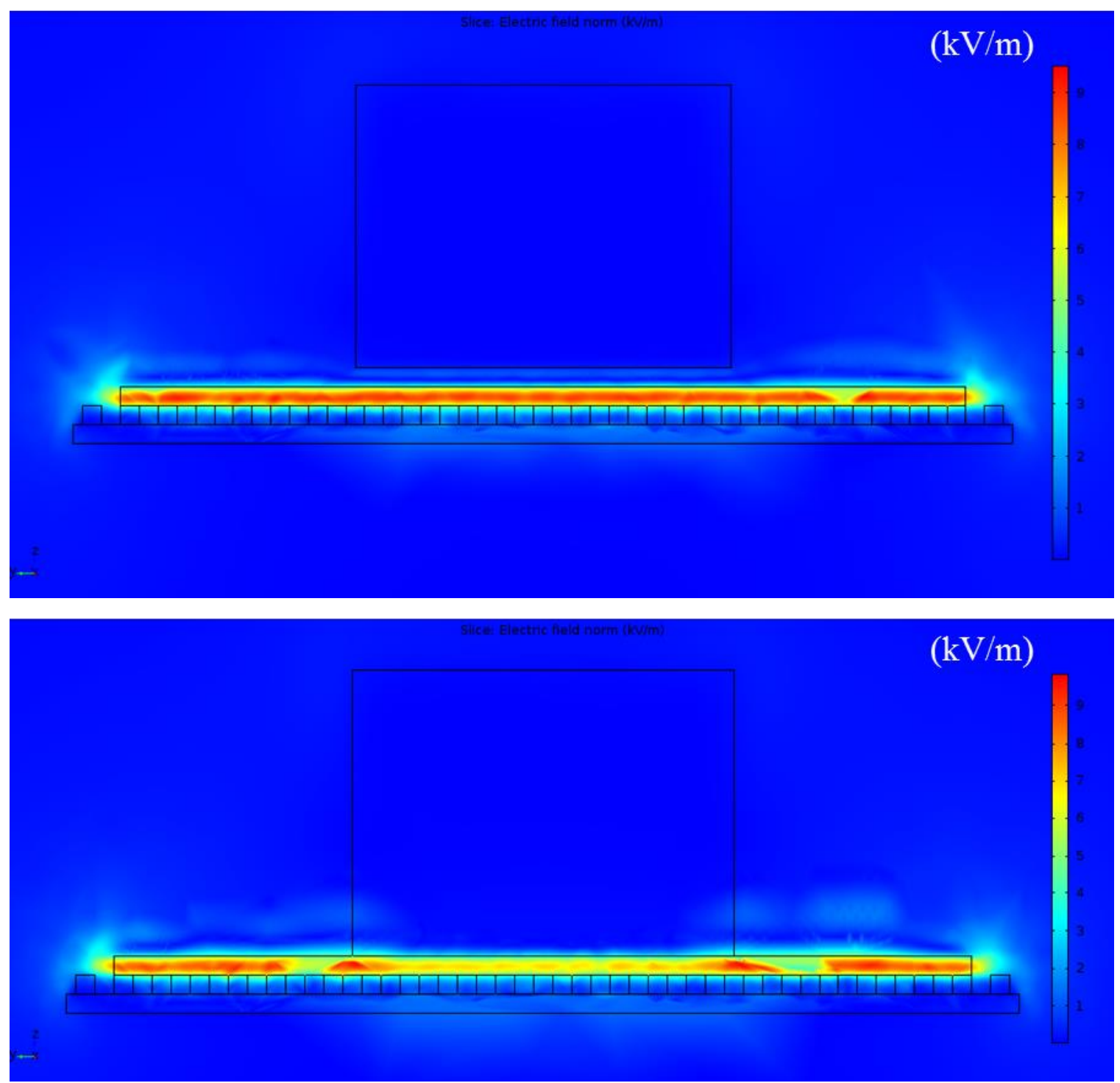

Figure 5.17 Simulations for a conducting object approaching sensor surface. Relative permittivity set to 10000 to simulate a conducting object in COMSOL. a) No contact with sensor and a10 $\mu \mathrm{m}$ gap, b) contact with sensor. Relative permittivity set to 100 to simulate a conducting object in COMSOL.

d) No contact with sensor and a10 $\mu \mathrm{m}$ gap, and e) contact with sensor. 

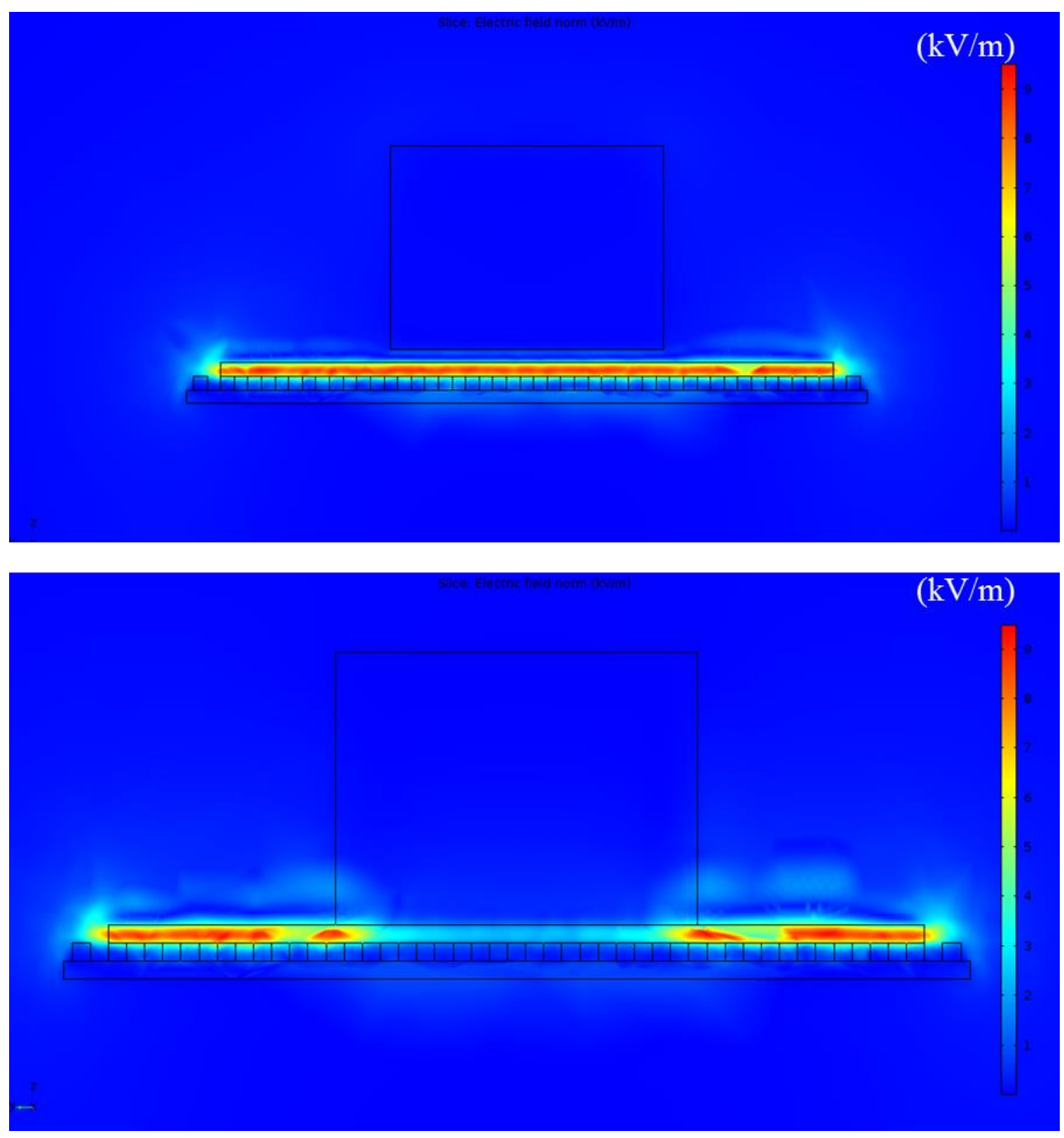

Figure 5.18 Simulations for a dielectric object approaching sensor surface. Relative permittivity set to 10: a) No contact with sensor and a10 $\mu \mathrm{m}$ gap, b) contact with sensor.

The initial capacitance as a function of the top object is reported in Figure 5.19. The symbol $C$ denotes contacting object and $N C$ a non-contacting object. The numerical values in the horizontal axis label denote the material permittivities used in COMSOL to simulate a dielectric or conducting object. Figure 5.20 shows the normalized initial 
capacitance relative to the sensor only capacitance. The results quantify the effect of the fringe field disturbance on the calculated capacitance. Up to a $6 \%$ change in capacitance is observed, which is numerically comparable to the capacitance change attributed to pressure alone. Figure 5.21 shows the device capacitance calculated as a function of pressure in the presence of a fixed object. The results show how object presence increases sensor sensitivity at higher pressures, which is an unexpected result. This tentatively points to the external object as a design variable to improve sensor performance. This would require careful refinement to isolate parasitic (undesirable) environmental effects.

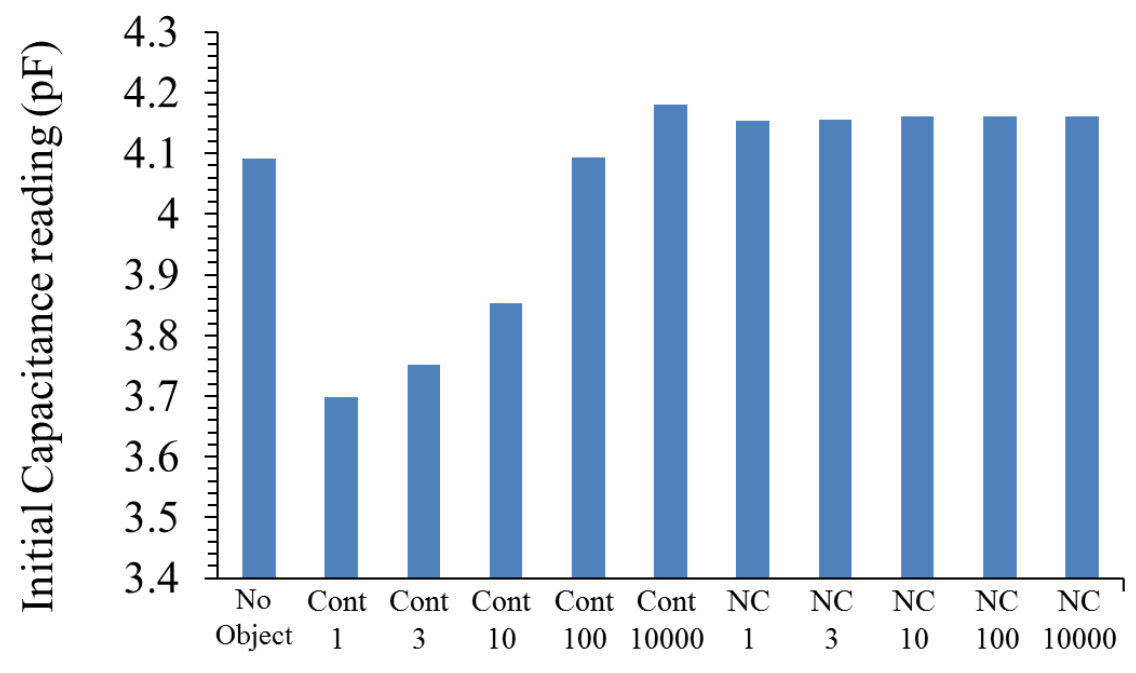

Figure 5.19 Initial sensor capacitance for each test case. 


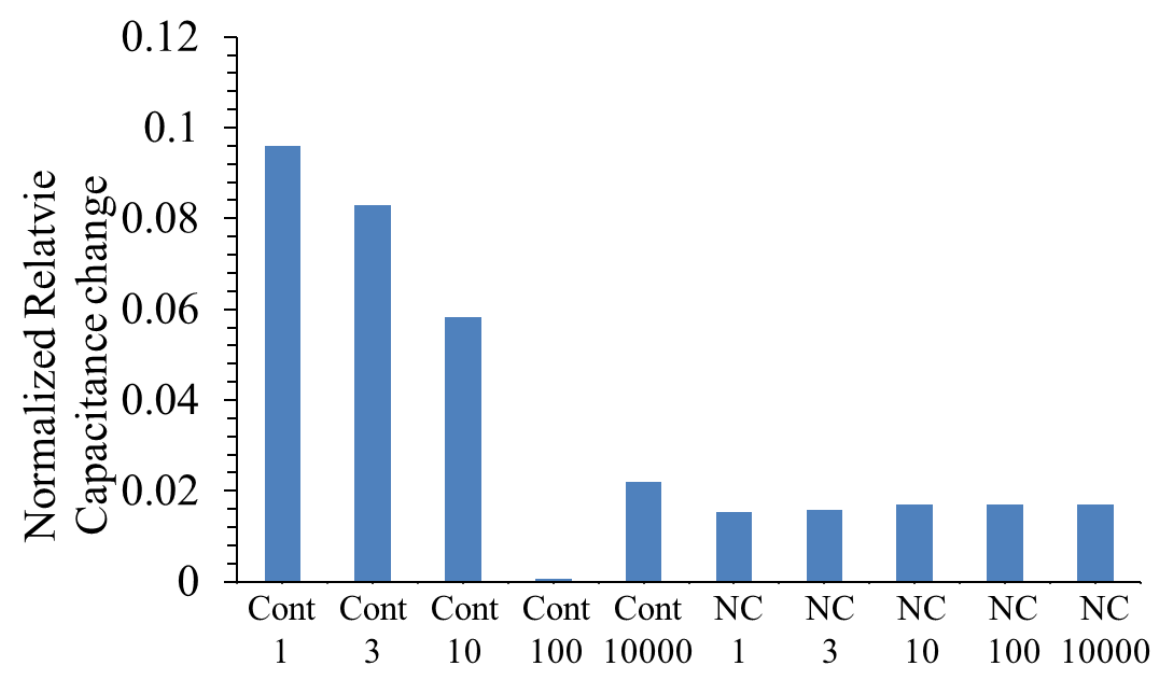

Figure 5.20 Relative capacitance change normalized with respect to sensor only capacitance.

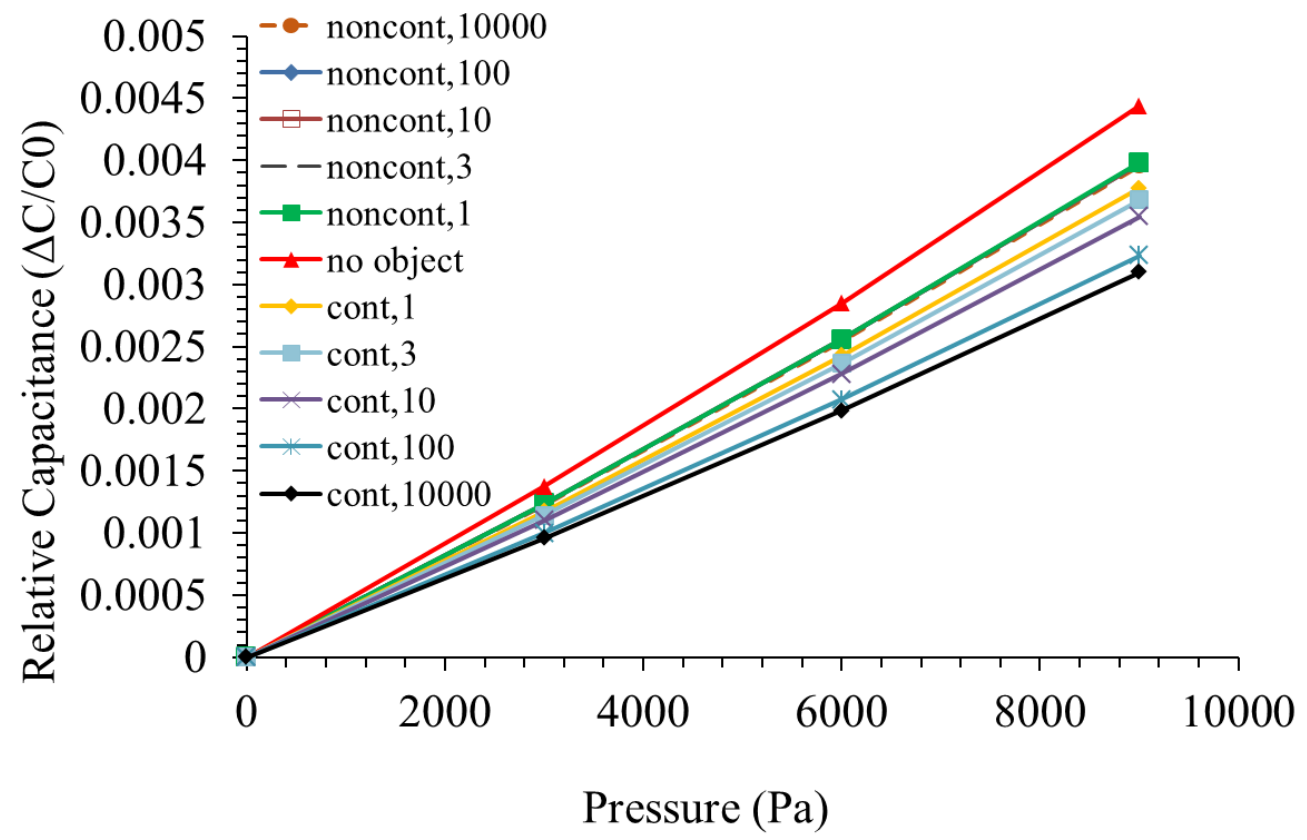

Figure 5.21 Relative capacitance change as a function of pressure in the presence of a dielectric or conducting object. 
Figure 5.22 shows the calculated sensor output for the ultra - low pressure regime 1-10 $\mathrm{Pa}$. The capacitance change is nearly zero suggesting that the sensor is not capable of detecting pressures in this regime. Extrapolating the results up to $100 \mathrm{~Pa}$ and analyzing the deformation behavior, we conclude that distinguishable pressure sensing occurs at approximately $80 \mathrm{~Pa}$ for the set of sensor designs fabricated.

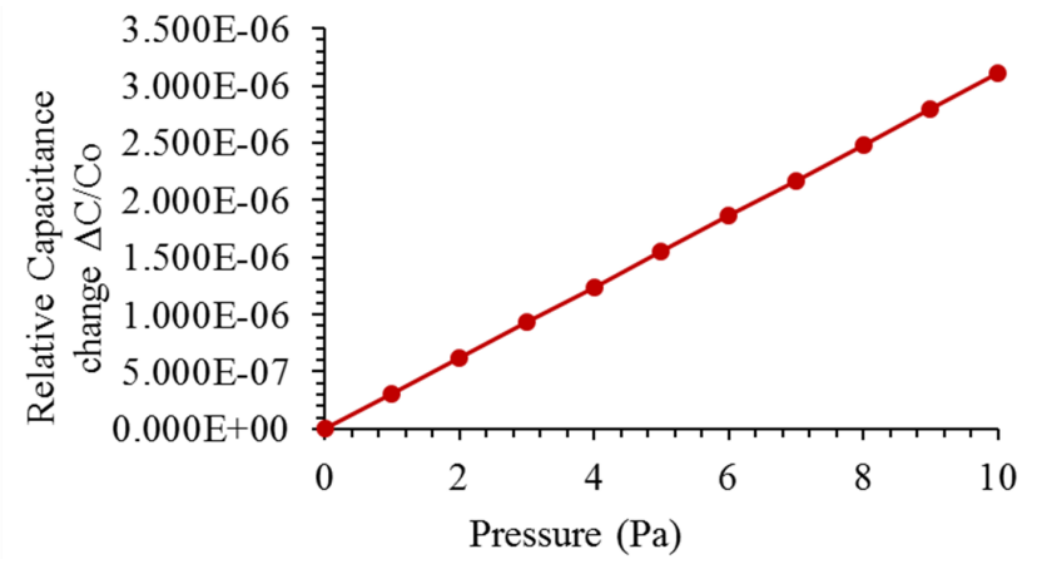

Figure 5.22. Relative capacitance change as a function of pressure in the 1-10 Pa range.

In the experimental tests, the pressure was applied to the sensor by placing stainless steel weights on the device. The simulation results show that it is important to isolate proximity sensing associated with the external fringe field from pressure sensing associated with changes to the internal electric field. Therefore, a threshold for appreciable device deformation should be established. These simulation results suggest that the experimental results (chapter 4) are due in part to proximity/presence sensing. Three points of careful note: i) The earlier simulations for pressure loads clearly show that by tailoring the design of the pillars large deformations can be achieved for a specific pressure range, which has a significant impact on the capacitance change. The coupled fringe field effects do not negate these findings. ii) There are well-known specially designed electrode configurations for proximity sensing if it is desirable to use the fringe field effect [63]. iii) There are existing solutions (electrical circuitry and sensor insulation) that can be employed to reduce parasitic capacitance and environmental effects $[87,88]$. In summary, these results place pressure sensor development at the 
interface with proximity sensor design considerations. This concept could however be explored further in future work to establish its viability in sensor design.

This chapter has shed light on the electromechanical complexities involved in highly deformable microstructured capacitive sensors and confirmed the experimental findings of Chapter 4. 


\section{Chapter 6}

\section{Summary and Conclusions}

\subsection{Summary}

In this research, the objective to achieve a skin level sensor system using existing fabrication techniques is accomplished. The soft Au/PDMS micropillar array is fabricated with soft lithography and electro-sputtering. The basic principle of sensor operation involves directly electroding the micropillars and forming a capacitive sensor configuration. This platform can easily be adapted for other functionalities such as flow sensing, resistive sensing, and actuation. Eighteen different designs are fabricated and analyzed using a combination of experiments and computational techniques. The pillar design variables explored are aspect ratio, gap distance, and cross-section shape. Fabrication methods for each design are optimized, which is critical for high aspect ratio

pillars. Techniques for fabricating asymmetric easy to buckle pillar structures and a multi-level hierarchical design platform are also developed. The fabrication method is simple and the fabrication error is within 5\%. Static and dynamic sensor characteristics are reported. The influence of pillar design parameters on sensor performance is explored using experimental and computational simulation techniques.

Electromechanical behavior of the micropillar sensor is modeled with the FEM software COMSOL 5.2. The coupled model aids in understanding the mechanism of capacitive sensing in micropillar arrays. The microstructured sensor array naturally lends itself to the development of pixel-type pressure sensors and biomedical monitoring devices - the 
potential for both applications are investigated here. The sensor array can be patterned to have fine spatial resolution, where discretizing the electrodes is the current limiting factor.

In summary, the micropillar sensor with the best performance was the hierarchical array. This sensor hybrid has a high sensitivity of $0.34 \mathrm{kPa}^{-1}$ and a broad pressure sensing range up to $10 \mathrm{kPa}$ with a single sensor platform (i.e. without modifying the structure). The sensing response is repeatable and the response time $(60.8 \mathrm{~ms})$ is comparably fast for capacitive sensors. Also, the system is flexible and stretchable up to $50 \%$ strain.

\subsection{Contributions}

1. A bio-inspired, microstructured pressure sensor is developed. The sensor has competitive performance with other flexible and stretchable systems and the fabrication method for the sensor is simple and promising.

2. Two novel micropillar designs are developed. One design takes advantage of a buckling mode instability. The second design is a hybrid design that uses a hierarchical array of high aspect ratio pillars to induce a prescriptive multi-level deformation as the pressure loading is increased. In Table 6.1, a detailed comparison with other pressure sensor work is given. The final row denotes the contribution from this thesis. 
Table 6.1 Micropillar Sensor Summary

\begin{tabular}{|c|c|c|c|c|c|c|}
\hline Type & Structure & $\begin{array}{l}\text { Sensing } \\
\text { mechanism }\end{array}$ & $\begin{array}{c}\text { Fabrication } \\
\text { technique }\end{array}$ & $\begin{array}{c}\text { Sensitivity } \\
\left(\mathrm{kPa}^{-1}\right)\end{array}$ & Range & $\begin{array}{c}\text { Response } \\
\text { time }\end{array}$ \\
\hline Flexible & $\begin{array}{l}\text { Gold serpentine } \\
\text { pattern on PDMS }\end{array}$ & Resistive & $\begin{array}{c}\text { PDMS molding } \\
\text { with Al mold }\end{array}$ & 0.23 & $\begin{array}{c}1 \mathrm{~Pa} \sim \\
6.7 \mathrm{kPa}\end{array}$ & $60 \mathrm{~ms}$ \\
\hline Flexible & $\begin{array}{l}\text { OFETs - indium tin } \\
\text { oxide (ITO)-coated } \\
\text { flexible PET sheet } \\
\text { on top of } \\
\text { microstructured } \\
\text { PDMS }\end{array}$ & Capacitive & $\begin{array}{c}\text { Photolith. for SI } \\
\text { mold, PVD of } \\
\text { Rubrene }\end{array}$ & $\begin{array}{c}0.55 \\
(<2 \mathrm{kPa} \\
\text { regime })\end{array}$ & $\begin{array}{l}3 \mathrm{~Pa} \sim \\
7 \mathrm{kPa}\end{array}$ & $\sim 200 \mathrm{~ms}$ \\
\hline Flexible & $\begin{array}{l}\text { Ppy gel (conducting } \\
\text { polymer) }\end{array}$ & Piezo-Resistive & $\begin{array}{c}\text { Micropatterned } \\
\text { inverse mold }\end{array}$ & $\begin{array}{l}56-133 \\
(<30 \mathrm{~Pa} \\
\text { regime })\end{array}$ & $\begin{array}{c}0.8 \mathrm{~Pa} \sim \\
10 \mathrm{kPa}\end{array}$ & $50 \mathrm{~ms}$ \\
\hline Flexible & $\begin{array}{c}\text { Reversible } \\
\text { interlocking of } \\
\text { nanoofibres }\end{array}$ & Piezo-Resistive & $\begin{array}{l}\text { SI Master, PUA } \\
\text { mold }\end{array}$ & $\begin{array}{l}11.45 \\
\text { (Gauge } \\
\text { factor) }\end{array}$ & $\begin{array}{l}5 \mathrm{~Pa} \sim \\
1.5 \mathrm{kPa}\end{array}$ & N/A \\
\hline Rigid & $\begin{array}{l}\text { parylene-based } \\
\text { wireless pressure } \\
\text { sensor }\end{array}$ & $\begin{array}{c}\text { Capacitor/indu } \\
\text { ctor resonant } \\
\text { circuits }\end{array}$ & $\begin{array}{c}\text { Silicon based } \\
\text { micromachined } \\
\text { (DRIE) }\end{array}$ & 0.058 & $\begin{array}{c}100 \mathrm{~Pa} \sim \\
4 \mathrm{kPa}\end{array}$ & N/A \\
\hline Rigid & $\begin{array}{c}\text { micropillar } \\
\text { structure on } \\
\text { conductive PPy } \\
\text { film } \\
\end{array}$ & Resistive & $\begin{array}{l}\text { PDMS molding } \\
\text { with SI mold }\end{array}$ & $0.03-17$ & $\begin{array}{l}2 \mathrm{~Pa} \sim \\
3 \mathrm{kPa}\end{array}$ & $\sim 1000 \mathrm{~ms}$ \\
\hline $\begin{array}{l}\text { Stretchable } \\
\text { up to } 150 \%\end{array}$ & $\begin{array}{c}\text { Stretched CNT on } \\
\text { PDMS }\end{array}$ & $\begin{array}{c}\text { Capacitive and } \\
\text { Resistive }\end{array}$ & $\begin{array}{c}\text { Spray coating of } \\
\text { CNT, multiple } \\
\text { layer stack }\end{array}$ & $2.3 \times 10^{-6}$ & $50 \mathrm{kPa} \sim$ & $<125 \mathrm{~ms}$ \\
\hline Flexible & $\begin{array}{c}\text { polymer } \\
\text { semiconductor/PD } \\
\text { MS dielectric }\end{array}$ & Capacitive & \begin{tabular}{|c|} 
assembling \\
separate layers \\
via lamination
\end{tabular} & $\begin{array}{c}8.4 \\
(<8 \mathrm{kPa})\end{array}$ & $50 \mathrm{~Pa} \sim$ & $<10 \mathrm{~ms}$ \\
\hline $\begin{array}{l}* \text { Flexible } \\
\text { and } \\
\text { Stretchable } \\
\text { up to } 50 \%\end{array}$ & $\begin{array}{c}\text { Au coated micro } \\
\text { pillar structure on } \\
\text { PDMS film }\end{array}$ & Capacitive & $\begin{array}{c}\text { Soft } \\
\text { lithography }\end{array}$ & 0.34 & $\begin{array}{l}80 \mathrm{~Pa} \sim \\
10 \mathrm{kPa}\end{array}$ & 60.8 \\
\hline
\end{tabular}

* The current thesis research results reported here. 
3. The relationship between micropillar design and sensing performance is determined. Collectively, the results clearly show how the reconfiguration of the dielectric media and electrode surfaces can lead to alterations in the nonuniform electric field. These three dimensional field alterations cause large changes in capacitance, and by extension increased sensitivity and pressure sensing range. It is clearly evidenced how pillar geometry can be tailored to optimize sensor performance.

4. Multiphysics simulations successfully conducted in COMSOL show identical trends to the experimental results. Specifically, increasing the aspect ratio and employing pillar geometries designed to buckle dramatically increase sensor sensitivity. Increasing gap distance can also improve sensitivity. To a smaller extent, pillar cross-section (for constant area) can influence the sensitivity as well.

5. The coupled simulation results show that it is important to isolate proximity sensing associated with the external fringe field from pressure sensing associated with changes to the internal electric field. Therefore, a threshold for appreciable device deformation should be established. These simulation results suggest that the experimental results in this thesis are due in part to proximity/presence sensing. 


\section{Bibliography}

1. Choong, Chwee-Lin, Mun-Bo Shim, Byoung-Sun Lee, Sanghun Jeon, Dong-Su Ko, Tae-Hyung Kang, Jihyun Bae, et al. "Highly Stretchable Resistive Pressure Sensors Using a Conductive Elastomeric Composite on a Micropyramid Array." Advanced Materials 26, no. 21 (June 1, 2014): 3451-58. doi:10.1002/adma.201305182.

2. Chun, Kyoung-Yong, Youngseok Oh, Jonghyun Rho, Jong-Hyun Ahn, Young-Jin Kim, Hyouk Ryeol Choi, and Seunghyun Baik. "Highly Conductive, Printable and Stretchable Composite Films of Carbon Nanotubes and Silver." Nature Nanotechnology 5, no. 12 (2010): 853-57. doi:10.1038/nnano.2010.232.

3. Jeong, Seung Hee, Klas Hjort, and Zhigang Wu. "Tape Transfer Atomization Patterning of Liquid Alloys for Microfluidic Stretchable Wireless Power Transfer." Scientific Reports 5 (February 12, 2015): 8419. doi:10.1038/srep08419.

4. Keplinger, Christoph, Jeong-Yun Sun, Choon Chiang Foo, Philipp Rothemund, George M. Whitesides, and Zhigang Suo. "Stretchable, Transparent, Ionic Conductors." Science 341, no. 6149 (August 30, 2013): 984-87.

doi:10.1126/science. 1240228.

5. Kim, Dae-Hyeong, Jianliang Xiao, Jizhou Song, Yonggang Huang, and John A. Rogers. "Stretchable, Curvilinear Electronics Based on Inorganic Materials." Advanced Materials 22, no. 19 (May 18, 2010): 2108-24. doi:10.1002/adma.200902927.

6. Lacour, S. P., J. Jones, S. Wagner, Teng Li, and Zhigang Suo. "Stretchable Interconnects for Elastic Electronic Surfaces." Proceedings of the IEEE 93, no. 8 (August 2005): 1459-67. doi:10.1109/JPROC.2005.851502.

7. Yamada, Takeo, Yuhei Hayamizu, Yuki Yamamoto, Yoshiki Yomogida, Ali IzadiNajafabadi, Don N. Futaba, and Kenji Hata. "A Stretchable Carbon Nanotube Strain Sensor for Human-Motion Detection." Nature Nanotechnology 6, no. 5 (2011): 296301. doi:10.1038/nnano.2011.36.

8. Yao, Shanshan, and Yong Zhu. "Wearable Multifunctional Sensors Using Printed Stretchable Conductors Made of Silver Nanowires." Nanoscale 6, no. 4 (January 30, 2014): 2345-52. doi:10.1039/C3NR05496A.

9. Schwartz, Gregor, Benjamin C.-K. Tee, Jianguo Mei, Anthony L. Appleton, Do Hwan Kim, Huiliang Wang, and Zhenan Bao. "Flexible Polymer Transistors with High Pressure Sensitivity for Application in Electronic Skin and Health Monitoring." Nature Communications 4 (May 14, 2013): 1859. doi:10.1038/ncomms2832.

10. Kramer, R. K., C. Majidi, and R. J. Wood. "Wearable Tactile Keypad with Stretchable Artificial Skin." In 2011 IEEE International Conference on Robotics and Automation (ICRA), 1103-7, 2011. doi:10.1109/ICRA.2011.5980082. 
11. Kramer, R. K., C. Majidi, and R. J. Wood. "Wearable Tactile Keypad with Stretchable Artificial Skin.” In 2011 IEEE International Conference on Robotics and Automation (ICRA), 1103-7, 2011. doi:10.1109/ICRA.2011.5980082.

12. Russomanno, A., S. O'Modhrain, R. B. Gillespie, and M. W. M. Rodger. "Refreshing Refreshable Braille Displays.” IEEE Transactions on Haptics 8, no. 3 (July 2015): 287-97. doi:10.1109/TOH.2015.2423492.

13. Tiwana, Mohsin I., Stephen J. Redmond, and Nigel H. Lovell. "A Review of Tactile Sensing Technologies with Applications in Biomedical Engineering." Sensors and Actuators A: Physical 179 (June 2012): 17-31. doi:10.1016/j.sna.2012.02.051.

14. Chang, Wen-Yang, Te-Hua Fang, Shao-Hsing Yeh, and Yu-Cheng Lin. "Flexible Electronics Sensors for Tactile Multi-Touching." Sensors 9, no. 2 (February 24, 2009): 1188-1203. doi:10.3390/s9021188.

15. Nicholls, Howard R., and Mark H. Lee. "A Survey of Robot Tactile Sensing Technology." The International Journal of Robotics Research 8, no. 3 (June 1, 1989): 3-30. doi:10.1177/027836498900800301.

16. Yousef, Hanna, Mehdi Boukallel, and Kaspar Althoefer. "Tactile Sensing for Dexterous in-Hand Manipulation in robotics-A Review." Sensors and Actuators A: Physical, Solid-State Sensors, Actuators and Microsystems Workshop, 167, no. 2 (June 2011): 171-87. doi:10.1016/j.sna.2011.02.038.

17. Hirokawa, Nobutaka, Yosuke Tanaka, Yasushi Okada, and Sen Takeda. "Nodal Flow and the Generation of Left-Right Asymmetry." Cell 125, no. 1 (April 7, 2006): 33-45. doi:10.1016/j.cell.2006.03.002.

18. C Brennen, and and H. Winet. "Fluid Mechanics of Propulsion by Cilia and Flagella." Annual Review of Fluid Mechanics 9, no. 1 (1977): 339-98. doi:10.1146/annurev.fl.09.010177.002011.

19. "Index: Mammalian Lung SEM." Accessed June 12, 2016. http://remf.dartmouth.edu/images/mammalianLungSEM/.

20. Liu, Kesong, Xi Yao, and Lei Jiang. "Recent Developments in Bio-Inspired Special Wettability." Chemical Society Reviews 39, no. 8 (July 22, 2010): 3240-55. doi:10.1039/B917112F.

21. Vogel, Nicolas, Stefanie Utech, Grant T. England, Tanya Shirman, Katherine R. Phillips, Natalie Koay, Ian B. Burgess, Mathias Kolle, David A. Weitz, and Joanna Aizenberg. "Color from Hierarchy: Diverse Optical Properties of Micron-Sized Spherical Colloidal Assemblies." Proceedings of the National Academy of Sciences 112, no. 35 (September 1, 2015): 10845-50. doi:10.1073/pnas.1506272112.

22. Kim, Hyung-Jung, Sung-Hyuk Song, and Sung-Hoon Ahn. "A Turtle-like Swimming Robot Using a Smart Soft Composite (SSC) Structure.” Smart Materials and Structures 22, no. 1 (January 1, 2013): 14007. doi:10.1088/0964-1726/22/1/014007.

23. Chandra, Dinesh, Shu Yang, Andre A. Soshinsky, and Robert J. Gambogi. "Biomimetic Ultrathin Whitening by Capillary-Force-Induced Random Clustering of Hydrogel Micropillar Arrays." ACS Applied Materials \& Interfaces 1, no. 8 (August 26, 2009): 1698-1704. doi:10.1021/am900253z.

24. Pan, Zhen, Ce Yan, Rong Peng, Yingchun Zhao, Yao He, and Jiandong Ding. "Control of Cell Nucleus Shapes via Micropillar Patterns." Biomaterials 33, no. 6 (February 2012): 1730-35. doi:10.1016/j.biomaterials.2011.11.023. 
25. Liu, Chang. "Micromachined Biomimetic Artificial Haircell Sensors." Bioinspiration \& Biomimetics 2, no. 4 (December 2007): S162-69. doi:10.1088/1748-3182/2/4/S05.

26. Lee, Haeshin, Bruce P. Lee, and Phillip B. Messersmith. "A Reversible Wet/dry Adhesive Inspired by Mussels and Geckos.” Nature 448, no. 7151 (July 19, 2007): 338-41. doi:10.1038/nature05968.

27. Qin, Dong, Younan Xia, and George M. Whitesides. "Soft Lithography for Micro- and Nanoscale Patterning." Nature Protocols 5, no. 3 (March 2010): 491-502. doi:10.1038/nprot.2009.234.

28. Shao, Qi, Zhiqiang Niu, Michael Hirtz, Lin Jiang, Yuanjun Liu, Zhaohui Wang, and Xiaodong Chen. "High-Performance and Tailorable Pressure Sensor Based on Ultrathin Conductive Polymer Film.” Small 10, no. 8 (April 1, 2014): 1466-72. doi:10.1002/smll.201303601.

29. Melchels, Ferry P. W., Jan Feijen, and Dirk W. Grijpma. "A Review on Stereolithography and Its Applications in Biomedical Engineering." Biomaterials 31, no. 24 (August 2010): 6121-30. doi:10.1016/j.biomaterials.2010.04.050.

30. Kang, Sung H., Ning Wu, Alison Grinthal, and Joanna Aizenberg. "Meniscus Lithography: Evaporation-Induced Self-Organization of Pillar Arrays into Moiré Patterns." Physical Review Letters 107, no. 17 (October 20, 2011): 177802. doi:10.1103/PhysRevLett.107.177802.

31. Kim, Philseok, Wilmer E. Adorno-Martinez, Mughees Khan, and Joanna Aizenberg. "Enriching Libraries of High-Aspect-Ratio Micro- or Nanostructures by Rapid, LowCost, Benchtop Nanofabrication." Nature Protocols 7, no. 2 (February 2012): 311-27. doi:10.1038/nprot.2012.003.

32. Zarzar, Lauren D., and Joanna Aizenberg. "Stimuli-Responsive Chemomechanical Actuation: A Hybrid Materials Approach." Accounts of Chemical Research, November 27, 2013. doi:10.1021/ar4001923.

33. Pang, Changhyun, Gil-Yong Lee, Tae-il Kim, Sang Moon Kim, Hong Nam Kim, Sung-Hoon Ahn, and Kahp-Yang Suh. "A Flexible and Highly Sensitive Strain-Gauge Sensor Using Reversible Interlocking of Nanofibres." Nature Materials 11, no. 9 (September 2012): 795-801. doi:10.1038/nmat3380.

34. Cheung, C. L., R. J. Nikolić, C. E. Reinhardt, and T. F. Wang. "Fabrication of Nanopillars by Nanosphere Lithography." Nanotechnology 17, no. 5 (March 14, 2006): 1339. doi:10.1088/0957-4484/17/5/028.

35. Li, Xiao, Yu-Hsuan Wang, Chen Zhao, and Xinyu Liu. "Paper-Based Piezoelectric Touch Pads with Hydrothermally Grown Zinc Oxide Nanowires.” ACS Applied Materials \& Interfaces 6, no. 24 (December 24, 2014): 22004-12. doi:10.1021/am504903b.

36. Mo, Yufei, Fuchuan Huang, Shulin Pan, and Shizhao Huang. "Fabrication and Adhesion Measurement of a Nanotextured Surface with Ionic Liquid Monolayers." The Journal of Physical Chemistry C 115, no. 20 (May 26, 2011): 10125-30. doi:10.1021/jp201285h.

37. Chen, P. J., D. C. Rodger, S. Saati, M. S. Humayun, and Y. C. Tai. "Microfabricated Implantable Parylene-Based Wireless Passive Intraocular Pressure Sensors." Journal of Microelectromechanical Systems 17, no. 6 (December 2008): 1342-51. doi:10.1109/JMEMS.2008.2004945. 
38. Lacour, S. P., J. Jones, S. Wagner, Teng Li, and Zhigang Suo. "Stretchable Interconnects for Elastic Electronic Surfaces." Proceedings of the IEEE 93, no. 8 (August 2005): 1459-67. doi:10.1109/JPROC.2005.851502.

39. Urdaneta, M. G., R. Delille, and E. Smela. "Stretchable Electrodes with High Conductivity and Photo-Patternability." Advanced Materials 19, no. 18 (September 17, 2007): 2629-33. doi:10.1002/adma.200601808.

40. Chae, Sang Hoon, and Young Hee Lee. "Carbon Nanotubes and Graphene towards Soft Electronics.” Nano Convergence 1, no. 1 (April 25, 2014): 1-26. doi:10.1186/s40580-014-0015-5.

41. Lipomi, Darren J., Michael Vosgueritchian, Benjamin C.-K. Tee, Sondra L. Hellstrom, Jennifer A. Lee, Courtney H. Fox, and Zhenan Bao. "Skin-like Pressure and Strain Sensors Based on Transparent Elastic Films of Carbon Nanotubes." Nature Nanotechnology 6, no. 12 (2011): 788-92. doi:10.1038/nnano.2011.184.

42. Fox, J. W., and N. C. Goulbourne. "On the Dynamic Electromechanical Loading of Dielectric Elastomer Membranes." Journal of the Mechanics and Physics of Solids 56, no. 8 (August 2008): 2669-86. doi:10.1016/j.jmps.2008.03.007.

43. Park, Junyong, Shuodao Wang, Ming Li, Changui Ahn, Jerome K. Hyun, Dong Seok Kim, Do Kyung Kim, John A. Rogers, Yonggang Huang, and Seokwoo Jeon. "ThreeDimensional Nanonetworks for Giant Stretchability in Dielectrics and Conductors." Nature Communications 3 (June 26, 2012): 916. doi:10.1038/ncomms1929.

44. Fiorini, Gina S., and Daniel T. Chiu. "Disposable Microfluidic Devices: Fabrication, Function, and Application." BioTechniques 38, no. 3 (March 2005): 429-46.

45. Kim, Jaemin, Mincheol Lee, Hyung Joon Shim, Roozbeh Ghaffari, Hye Rim Cho, Donghee Son, Yei Hwan Jung, et al. "Stretchable Silicon Nanoribbon Electronics for Skin Prosthesis." Nature Communications 5 (December 9, 2014): 5747. doi:10.1038/ncomms6747.

46. Smith, A. D., F. Niklaus, A. Paussa, S. Vaziri, A. C. Fischer, M. Sterner, F. Forsberg, et al. "Electromechanical Piezoresistive Sensing in Suspended Graphene Membranes." Nano Letters 13, no. 7 (July 10, 2013): 3237-42. doi:10.1021/n1401352k.

47. Takei, Kuniharu, Toshitake Takahashi, Johnny C. Ho, Hyunhyub Ko, Andrew G. Gillies, Paul W. Leu, Ronald S. Fearing, and Ali Javey. "Nanowire Active-Matrix Circuitry for Low-Voltage Macroscale Artificial Skin.” Nature Materials 9, no. 10 (2010): 821-26. doi:10.1038/nmat2835.

48. Kramer, R. K., C. Majidi, and R. J. Wood. "Wearable Tactile Keypad with Stretchable Artificial Skin.” In 2011 IEEE International Conference on Robotics and Automation (ICRA), 1103-7, 2011. doi:10.1109/ICRA.2011.5980082.

49. Du, Jinhong, Songfeng Pei, Laipeng Ma, and Hui-Ming Cheng. "25th Anniversary Article: Carbon Nanotube- and Graphene-Based Transparent Conductive Films for Optoelectronic Devices." Advanced Materials, March 1, 2014, n/a-n/a. doi:10.1002/adma.201304135.

50. Frank, Otakar, Georgia Tsoukleri, Ibtsam Riaz, Konstantinos Papagelis, John Parthenios, Andrea C. Ferrari, Andre K. Geim, Kostya S. Novoselov, and Costas Galiotis. "Development of a Universal Stress Sensor for Graphene and Carbon Fibres." Nature Communications 2 (March 29, 2011): 255. doi:10.1038/ncomms 1247. 
51. Mannsfeld, Stefan C. B., Benjamin C.-K. Tee, Randall M. Stoltenberg, Christopher V. H.-H. Chen, Soumendra Barman, Beinn V. O. Muir, Anatoliy N. Sokolov, Colin Reese, Zhenan Bao, and (first). "Highly Sensitive Flexible Pressure Sensors with Microstructured Rubber Dielectric Layers." Nature Materials 9, no. 10 (2010): 859-64. doi:10.1038/nmat2834.

52. Gong, Shu, Willem Schwalb, Yongwei Wang, Yi Chen, Yue Tang, Jye Si, Bijan Shirinzadeh, and Wenlong Cheng. "A Wearable and Highly Sensitive Pressure Sensor with Ultrathin Gold Nanowires." Nature Communications 5 (February 4, 2014): 3132. doi:10.1038/ncomms4132.

53. Park, Minwoo, Jungkyun Im, Minkwan Shin, Yuho Min, Jaeyoon Park, Heesook Cho, Soojin Park, et al. "Highly Stretchable Electric Circuits from a Composite Material of Silver Nanoparticles and Elastomeric Fibres.” Nature Nanotechnology 7, no. 12 (December 2012): 803-9. doi:10.1038/nnano.2012.206.

54. Charalambides, A., and S. Bergbreiter. "All-Elastomer in-Plane MEMS Capacitive Tactile Sensor for Normal Force Detection.” In 2013 IEEE SENSORS, 1-4, 2013. doi:10.1109/ICSENS.2013.6688461.

55. Codd, Patrick J., Arabagi Veaceslav, Andrew H. Gosline, and Pierre E. Dupont. "Novel Pressure-Sensing Skin for Detecting Impending Tissue Damage during Neuroendoscopy." Journal of Neurosurgery: Pediatrics 13, no. 1 (November 1, 2013): 114-21. doi:10.3171/2013.9.PEDS12595.

56. Jung, Taekeon, and Sung Yang. "Highly Stable Liquid Metal-Based Pressure Sensor Integrated with a Microfluidic Channel." Sensors 15, no. 5 (May 21, 2015): 11823-35. doi:10.3390/s150511823.

57. Kramer, R. K., C. Majidi, and R. J. Wood. "Wearable Tactile Keypad with Stretchable Artificial Skin." In 2011 IEEE International Conference on Robotics and Automation (ICRA), 1103-7, 2011. doi:10.1109/ICRA.2011.5980082.

58. Yao, Shanshan, and Yong Zhu. "Wearable Multifunctional Sensors Using Printed Stretchable Conductors Made of Silver Nanowires." Nanoscale 6, no. 4 (January 30, 2014): 2345-52. doi:10.1039/C3NR05496A.

59. Liu, Yang, Meng Gao, Shengfu Mei, Yanting Han, and Jing Liu. "Ultra-Compliant Liquid Metal Electrodes with in-Plane Self-Healing Capability for Dielectric Elastomer Actuators." Applied Physics Letters 103, no. 6 (August 5, 2013): 64101. doi:10.1063/1.4817977.

60. Pan, Lijia, Alex Chortos, Guihua Yu, Yaqun Wang, Scott Isaacson, Ranulfo Allen, Yi Shi, Reinhold Dauskardt, and Zhenan Bao. "An Ultra-Sensitive Resistive Pressure Sensor Based on Hollow-Sphere Microstructure Induced Elasticity in Conducting Polymer Film.” Nature Communications 5 (January 6, 2014): 3002. doi:10.1038/ncomms4002.

61. Pang, Changhyun, Gil-Yong Lee, Tae-il Kim, Sang Moon Kim, Hong Nam Kim, Sung-Hoon Ahn, and Kahp-Yang Suh. "A Flexible and Highly Sensitive Strain-Gauge Sensor Using Reversible Interlocking of Nanofibres." Nature Materials 11, no. 9 (September 2012): 795-801. doi:10.1038/nmat3380.

62. Tiwana, Mohsin I., Stephen J. Redmond, and Nigel H. Lovell. "A Review of Tactile Sensing Technologies with Applications in Biomedical Engineering." Sensors and Actuators A: Physical 179 (June 2012): 17-31. doi:10.1016/j.sna.2012.02.051. 
63. Baxter, Larry K. Capacitive Sensors: Design and Applications. John Wiley \& Sons, 1996.

64. Logothetidis, Stergios. Handbook of Flexible Organic Electronics: Materials, Manufacturing and Applications. Elsevier, 2014.

65. Tiwari, Atul, and Baldev Raj. Materials and Failures in MEMS and NEMS. John Wiley \& Sons, 2015.

66. Kuo, Alex CM. "Poly (dimethylsiloxane)." Polymer data handbook (1999): 411-435.

67. Gorman, Christopher B., Hans A. Biebuyck, and George M. Whitesides. "Fabrication of Patterned, Electrically Conducting Polypyrrole Using a Self-Assembled Monolayer: A Route to All-Organic Circuits." Chemistry of Materials 7, no. 3 (March 1, 1995): 526-29. doi:10.1021/cm00051a013.

68. Nix, William D. "Mechanical Properties of Thin Films." Metallurgical Transactions A 20, no. 11 (November 1989): 2217-45. doi:10.1007/BF02666659.

69. Begley, Matthew R., Hilary Bart-Smith, Orion N. Scott, Michael H. Jones, and Michael L. Reed. "The Electro-Mechanical Response of Elastomer Membranes Coated with Ultra-Thin Metal Electrodes." Journal of the Mechanics and Physics of Solids 53, no. 11 (November 2005): 2557-78. doi:10.1016/j.jmps.2005.05.002.

70. Volynskii, A. L., S. Bazhenov, O. V. Lebedeva, and N. F. Bakeev. "Mechanical Buckling Instability of Thin Coatings Deposited on Soft Polymer Substrates." Journal of Materials Science 35, no. 3 (February 2000): 547-54. doi:10.1023/A:1004707906821.

71. Venugopal, Gunasekaran, and Sang-Jae Kim. "Nanolithography." In Advances in Micro/Nano Electromechanical Systems and Fabrication Technologies, edited by Kenichi Takahata. InTech, 2013. http://www.intechopen.com/books/advances-inmicro-nano-electromechanical-systems-and-fabrication-technologies/nanolithography.

72. Li, Paul C. H. Microfluidic Lab-on-a-Chip for Chemical and Biological Analysis and Discovery. CRC Press, 2005.

73. Jackson, Mark J. Microfabrication and Nanomanufacturing. CRC Press, 2005.

74. Franssila, Sami. Introduction to Microfabrication. John Wiley \& Sons, 2010.

75. Stormonth-Darling, J. M., R. H. Pedersen, C. How, and N. Gadegaard. "Injection Moulding of Ultra High Aspect Ratio Nanostructures Using Coated Polymer Tooling." Journal of Micromechanics and Microengineering 24, no. 7 (2014): 75019. doi:10.1088/0960-1317/24/7/075019.

76. "Lithography Processing - LNF Wiki." Accessed June 12, 2016. http://lnfwiki.eecs.umich.edu/wiki/Lithography_processing.

77. Kang, Dae Y., Yun-Soung Kim, Gladys Ornelas, Mridu Sinha, Keerthiga Naidu, and Todd P. Coleman. "Scalable Microfabrication Procedures for Adhesive-Integrated Flexible and Stretchable Electronic Sensors." Sensors 15, no. 9 (September 16, 2015): 23459-76. doi:10.3390/s150923459.

78. Rogers, John A., Takao Someya, and Yonggang Huang. "Materials and Mechanics for Stretchable Electronics.” Science 327, no. 5973 (March 26, 2010): 1603-7. doi:10.1126/science.1182383.

79. "Lab 18-2 User Tool SOP." Google Docs. Accessed June 12, 2016. https://docs.google.com/document/d/1tXD5ABUT7U9eY8iZhgvfND5XGPfkHmby6 O6Tp-9bU1A/preview?pli=1\&usp=embed_facebook. 
80. "Lab 18-2 Characterization Old.” Google Docs. Accessed June 12, 2016. https://docs.google.com/spreadsheets/d/1oDatFum_LO6W0wmL_yhsiUbzpk5caKSqUNPuX3QvcY/edit?usp=embed_facebook.

81. Zhang, Ying, Chi-Wei Lo, J. Ashley Taylor, and Shu Yang. "Replica Molding of High-Aspect-Ratio Polymeric Nanopillar Arrays with High Fidelity." Langmuir 22, no. 20 (September 1, 2006): 8595-8601. doi:10.1021/la061372+.

82. "Dektak6M System Overview.” Google Docs. Accessed June 12, 2016. https://docs.google.com/document/d/1c2t5XLA-uvxuLe771qMx_cT9k8gNmbWQbmvjSRIMYs/preview?pli=1\&usp=embed_facebook.

83. "EVAL-AD7746 Evaluation Board | Analog Devices." Accessed June 12, 2016. http://www.analog.com/en/design-center/evaluation-hardware-andsoftware/evaluation-boards-kits/eval-ad7746.html\#eb-overview.

84. Yao, Shanshan, and Yong Zhu. "Wearable Multifunctional Sensors Using Printed Stretchable Conductors Made of Silver Nanowires." Nanoscale 6, no. 4 (January 30, 2014): 2345-52. doi:10.1039/C3NR05496A.

85. Ansari, S., N. Farzaneh, M. Heung, K. Oldham, H. Derksen, K. R. Ward, and K. Najarian. "Real-Time Detection of Intradialytic Hypotension Using a Novel Polyvinylidene Fluoride Based Sensor.” In 2016 IEEE-EMBS International Conference on Biomedical and Health Informatics (BHI), 493-96, 2016. doi:10.1109/BHI.2016.7455942.

86. Hosseini, Mehran, Guchuan Zhu, and Yves-Alain Peter. "A New Formulation of Fringing Capacitance and Its Application to the Control of Parallel-Plate Electrostatic Micro Actuators." Analog Integrated Circuits and Signal Processing 53, no. 2-3 (June 27, 2007): 119-28. doi:10.1007/s10470-007-9067-3.

87. Ko, Wen H., and Qiang Wang. "Touch Mode Capacitive Pressure Sensors." Sensors and Actuators A: Physical 75, no. 3 (June 8, 1999): 242-51. doi:10.1016/S09244247(99)00069-2.

88. Ko, W. H., Min-Hang Bao, and Yeun-Ding Hong. "A High-Sensitivity IntegratedCircuit Capacitive Pressure Transducer." IEEE Transactions on Electron Devices 29, no. 1 (January 1982): 48-56. doi:10.1109/T-ED.1982.20657.

89. Frank, Otakar, Georgia Tsoukleri, Ibtsam Riaz, Konstantinos Papagelis, John Parthenios, Andrea C. Ferrari, Andre K. Geim, Kostya S. Novoselov, and Costas Galiotis. "Development of a Universal Stress Sensor for Graphene and Carbon Fibres." Nature Communications 2 (March 29, 2011): 255. doi:10.1038/ncomms1247. 